Article

\title{
Phytosociology and Vegetation of Plants of Beit Jibrin in Palestine
}

\author{
Jehad Mahmoud Hussein Ighbareyeh ${ }^{1,2, * \mathbb{D}}$, Ana Cano-Ortiz ${ }^{2}$ and Eusebio Cano $^{2}$ (D)
}

1 Department of Plant Production and Protection, Faculty of Agriculture, Al-Quds Open University, Hebron Branch, Abu ktellah Street, Hebron P.O. Box 51800, Palestine

2 Department of Animal and Plant Biology and Ecology, Faculty of Experimental Sciences, University of Jaen, Paraje las Lagunillas s/n., 23071 Jaen, Spain; anacanor@hotmail.com (A.C.-O.); ecano@ujaen.es (E.C.)

* Correspondence: jighbareyeh@qou.edu; Tel.: +97-25-9999-1368

check for updates

Citation: Ighbareyeh, J.M.H.; Cano-Ortiz, A.; Cano, E.

Phytosociology and Vegetation of

Plants of Beit Jibrin in Palestine. Land 2022, 11, 264. https://doi.org/ 10.3390/land11020264

Academic Editor: Guillermo

J. Martinez-Pastur

Received: 24 November 2021

Accepted: 4 January 2022

Published: 10 February 2022

Publisher's Note: MDPI stays neutral with regard to jurisdictional claims in published maps and institutional affiliations.

Copyright: (C) 2022 by the authors. Licensee MDPI, Basel, Switzerland. This article is an open access article distributed under the terms and conditions of the Creative Commons Attribution (CC BY) license (https:// creativecommons.org/licenses/by/ $4.0 /)$.

\begin{abstract}
This paper describes a study on the vegetation and floristics of the territory of Beit Jibrin in Palestine, in areas such as Forest the Snabreh (Qasa), Khallet Mahmoud and Khallet AL-Taweel, among others. In view of the lack of studies on the phytosociology and communities of plants in the south-west of Palestine, as this region represents a unique diversity of plants, and the addition of these plants to Mediterranean Basin region plants, we conducted this study to identify and describe the plants of this region. Beit Jibrin is an ancient Canaanite Palestinian city that belongs to inframediterranean and thermomediterranean thermotypes, as well as arid, semi-arid and dry ombrotypes. This area is very important floristically, with a high rate of endemism: of the 290 species documented, 37 of them $(12.75 \%)$ were endemic to the region. Vegetation was sampled on twelve representative plots (releves) and analyzed using the Braun-Blanquet phytosociological analysis method. Two communities of forest maquis, macchie and steppe vegetation were found. Forest vegetation were represented by the Cupresso sempervirentis-Pinetum halepensis ass. nova. association, in the class of Quercetea ilicis Br.-Bl. ex. A. and O. Bolòs 1950, the order of Pinetalia halepensis, Biondi et al. (2014), and a new alliance: Cupresso sempervirentis-Pinus halepensis; forests maquis vegetation as the association of Pistacio lentisci-Quercetum calliprini ass. nova., with the suggested new class of Quercetea calliprini or palaestini in addition to Quercetea ilicis Br.-Bl. ex. A. and O. Bolòs 1950 and the order of Quercetalia calliprini (Zohary 1960), with an alliance of Quercion calliprini (Zohary 1960). These were adapted in arid, semi-arid, dry and sub-humid ombrotypes, as well as infra- and thermotropical to mesomediterranean thermotypes, with many different types of soils, such as limestone, brown ruinsenas, terra rossa and others.
\end{abstract}

Keywords: floristics; plant communities; taxonomy; ecology; associations

\section{Introduction}

Palestine is a hotspot for biodiversity and flora, and is considered one of the most biodiverse countries in West Asia and the Mediterranean coast. Palestinian coastal waters and mountain highlands possess a large level of biological diversity, in addition to many endemic and native species. Geographical and biological diversity is very important, consisting of landscapes and ecosystems that include areas of mountainous heights, plains, valleys, cliffs, sand dunes, steppes and forests. This prompted us to study the plant species of an important region located to the southwest of Palestine, west of the Jordan River and the Dead Sea, with the varieties of wild and forest plants it represents, as it is fertile and rich in forests of various plants. Given the lack of studies on plants' phytosociology or plant communities in this region, as well as its geographical, topographical, biological and biodiversity importance, it was necessary to work on studying the taxonomic, phytosociological and biological characteristics of plants and others. Ecological, climate change, climatic and bioclimate factors play an important role in plant distribution and biodiversity [1-3]. More than 2780 plant species have been studied, of which 162 species were endemic; 
872 genera and 144 families have been recorded for Palestinian flora [2-15]. Furthermore, some scientists have studied plant communities, the phytosociology of plants [16-18] and biodiversity in Palestine [19] in addition to the Mediterranean region [20-28]. The purpose of this paper is to study the phytosociology and plant taxa species of the Beit Jibrin region in the southwest of Palestine as well as of the Mediterranean Basin region, especially the eastern Mediterranean.

\section{Materials and Methods}

\subsection{Study Area}

Beit Jibrin (Jibreen) is a Palestinian Arab Canaanite village located $21 \mathrm{~km}$ northwest of Hebron and $13 \mathrm{~km}$ west of the village of Idna-Hebron, a wide area of hills, mountains and various valleys between the coastal plain to the west and the highland of Hebron to the east, where it is located within coordinates $\left(31^{\circ} 36^{\prime} 19^{\prime \prime} \mathrm{N}, 34^{\circ} 53^{\prime} 54^{\prime \prime} \mathrm{E}\right)$, with rises $275 \mathrm{~m}$ above sea level [29]. The total area of the village is 56,185 dunums $\left(56.1 \mathrm{~km}^{2}\right)$, of which $28 \mathrm{~km}^{2}$ are built-up whereas the rest remains as agricultural land [30,31]. Moreover, Beit Jibrin is characterized by the presence of many different archaeological caves (Caves 1000), which were included as a "United Nations Educational, Scientific and Cultural Organization" (UNESCO) World Heritage Site [32], near which there are many different plants, such as thyme, Pistachios spp., Rhamnus spp., R. palaestinus Boiss. and various herbal plants (Figure 1).

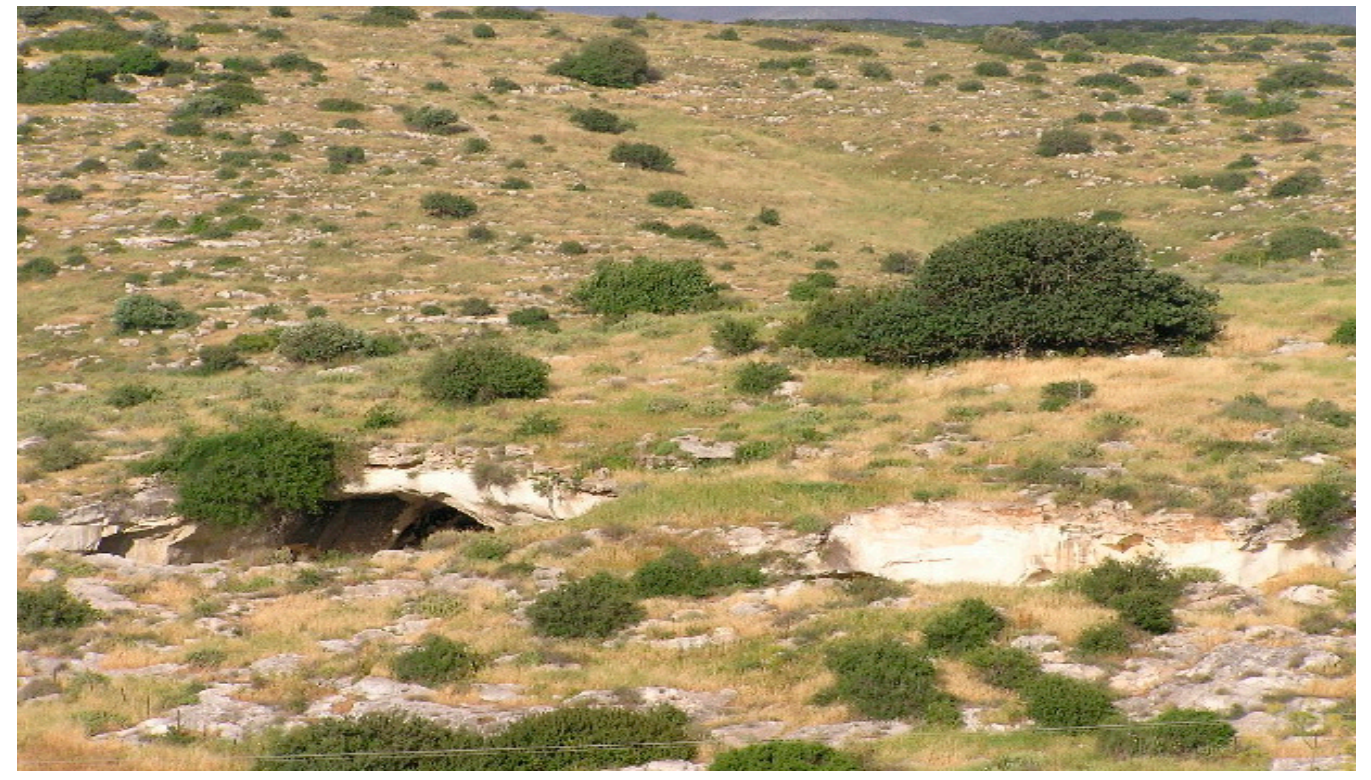

Figure 1. Vegetation in Beit Jibrin (Palestine).

\subsection{Vegetation Data Collection}

The study included the sampling area in Beit Jibrin, occupied since 1948, where the selection of and data collection of 290 woody plants took place, from Beit Jibrin forests or its hills, as well as some of the scrubland areas, to take biological and ecological indicators and plants for their traditional and thermal patterns (Table 1 and Figure 2A). These data were taken by using a Braun-Blanquet methodology [33,34]. We created a matrix of $290 \times 12$ related columns to convert the Braun-Blanquet plant phytosociological indicators $(+=2$, $1=3,2=4,3=5,4=6$ and $5=7$ ) into ones of Van der Maarel [35] (5: covering more than 3/4 of the area; 4 : any number of individuals covering $\frac{1}{2}-3 / 4$ of the area; 3 : any number of individuals covering $1 / 4-1 / 2$ of the area; 2 : very numerous or covering at least $5 \%$ of the area; and 1: plentiful but of small cover value, and + is a very small amount of cover) (Figure 2B). However, we used a phytosociological nomenclature code in the description of the new syntaxons in the study [36-38] and Euclidean distances as well 
as principal component analysis to evade any lack of data on whole-plant analysis; the XLSTAT Statistical Software for Excel program was used in the analysis process.

All the sites mentioned in the table are located in the area of Beit Jibreen and its surroundings - the name of Tal Sandhanh has been changed to Beit Guvrin-Maresha National Park.

The plants of Palestine, Syria, Lebanon, Jordan, Negev Desert, the Sinai desert and the Mediterranean coast were used in the study of flora, as cover vegetation of sites in the west of Hebron to the east of the occupied Palestinian coasts, such as Beer Sheva, Ashdod, Ashqelon (Asgalan or Al-Majdal), Lod, Ramle, Jaffa, Haifa, Safad, Acre, Iraq Mansheya and Al-Jalil, and neighboring villages, such as Ajjur, Beit Nir, Al-Dawaimah, Kidna, Faluja, Deir Ula, Qubeibeh, Zachariah and Idna [16-18], which form part of this adjacent plant environment. The vegetation has been explicated according to many methodological works, such as Braun-Blanquet and Bolòs [37], Bolòs [39], Oakley [40], Bolòs et al. [41], Pott [42], Biondi [43] and Rivas-Martinez et al. [44-51]. The west Hebron area has a dry climate, inframediterranean to thermomediterranean thermotype, with precipitation ranges between 250 and $550 \mathrm{~mm}$, and Beit Jibrin is a part of this area and the climate [1,18].

Table 1. Sampling regime.

\begin{tabular}{|c|c|c|c|c|}
\hline Inventories & Coordinates & Site & Altitude & Biogeographic Unit \\
\hline Inv. 1 & $31^{\circ} 34^{\prime} 31^{\prime \prime}$ & Forest the Snabreh (Qasa) & 325 & Mediterranean basin territories \\
\hline Inv. 2 & $31^{\circ} 34^{\prime} 34^{\prime \prime}$ & Forest Deir Nakhas & 285 & Mediterranean basin territories \\
\hline Inv. 3 & $31^{\circ} 34^{\prime} 40^{\prime \prime}$ & Khallet Mahmoud and Khallet Ataweel & 275 & Mediterranean basin territories \\
\hline Inv. 4 & $31^{\circ} 34^{\prime} 38^{\prime \prime}$ & $\begin{array}{c}\text { Khallet Deir Nakhas, Abu Rkheem and } \\
\text { Khallet Al-Moghiti }\end{array}$ & 270 & Mediterranean basin territories \\
\hline Inv. 5 & $31^{\circ} 34^{\prime} 45^{\prime \prime}$ & Al-Qagab & 260 & Mediterranean basin territories \\
\hline Inv. 6 & $31^{\circ} 34^{\prime} 42^{\prime \prime}$ & $\begin{array}{c}\text { Stoning of Azzami, Ganan Am-Ghamis and } \\
\text { Khallet Al-naje }\end{array}$ & 310 & Mediterranean basin territories \\
\hline Inv. 7 & $31^{\circ} 34^{\prime} 30^{\prime \prime}$ & Tal Sandhanh & 350 & Mediterranean basin territories \\
\hline Inv. 8 & $31^{\circ} 34^{\prime} 50^{\prime \prime}$ & Beit Jibrin Center & 300 & Mediterranean basin territories \\
\hline Inv. 9 & $31^{\circ} 34^{\prime} 33^{\prime \prime}$ & $\begin{array}{l}\text { Merhan Al-Motalah, Khirbit Am-Alahem and } \\
\text { Wadi Al-Arabe, Yardeh, Ain-Ismael Ayesh }\end{array}$ & 370 & Mediterranean basin territories \\
\hline Inv. 10 & $31^{\circ} 34^{\prime} 34^{\prime \prime}$ & $\begin{array}{l}\text { Wadi Abu Al Khail, Rasm Al-hajj Ahmad, } \\
\text { Wadi Algoga, Khallet Abu Jaber, Al-moalaga }\end{array}$ & 300 & Mediterranean basin territories \\
\hline Inv. 11 & $31^{\circ} 34^{\prime} 22^{\prime \prime}$ & Khallet Al-Qaisi and Wadi Al-sheikh Barak & 310 & Mediterranean basin territories \\
\hline Inv. 12 & $31^{\circ} 34^{\prime} 15^{\prime \prime}$ & Tal-Arbid, surrounding Beit Nair & 350 & Mediterranean basin territories \\
\hline
\end{tabular}

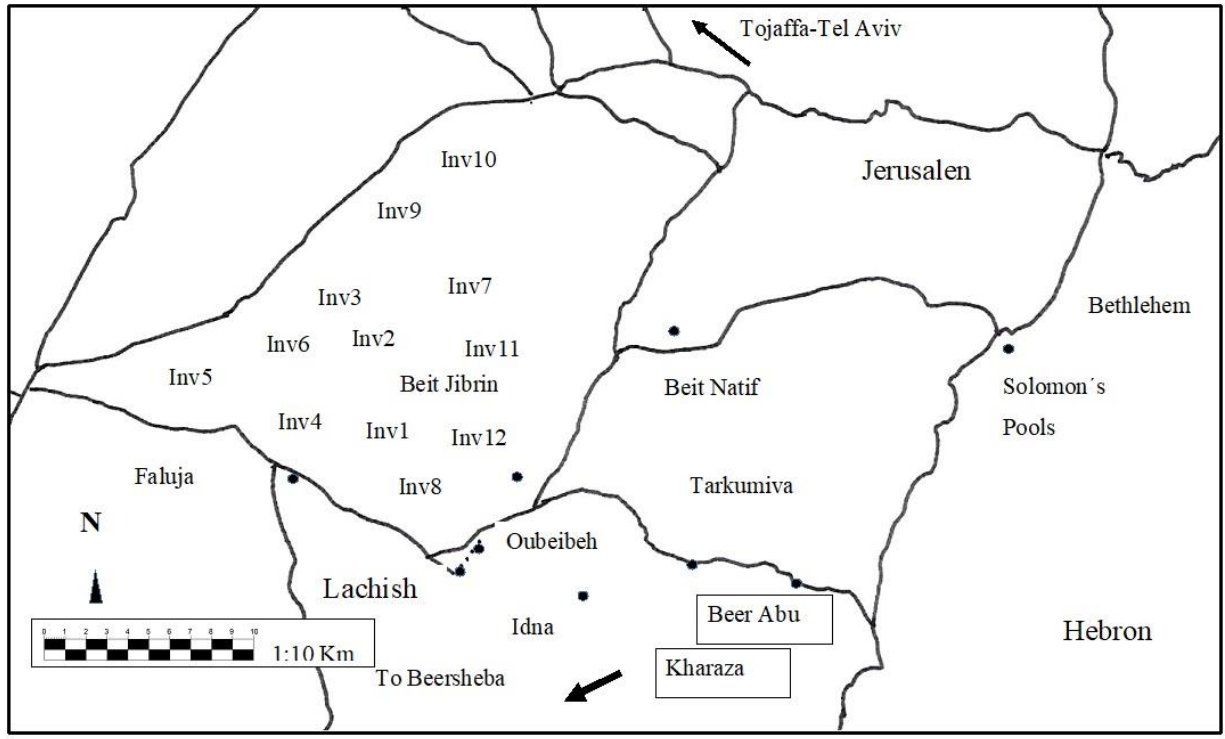

(A)

Figure 2. Cont. 


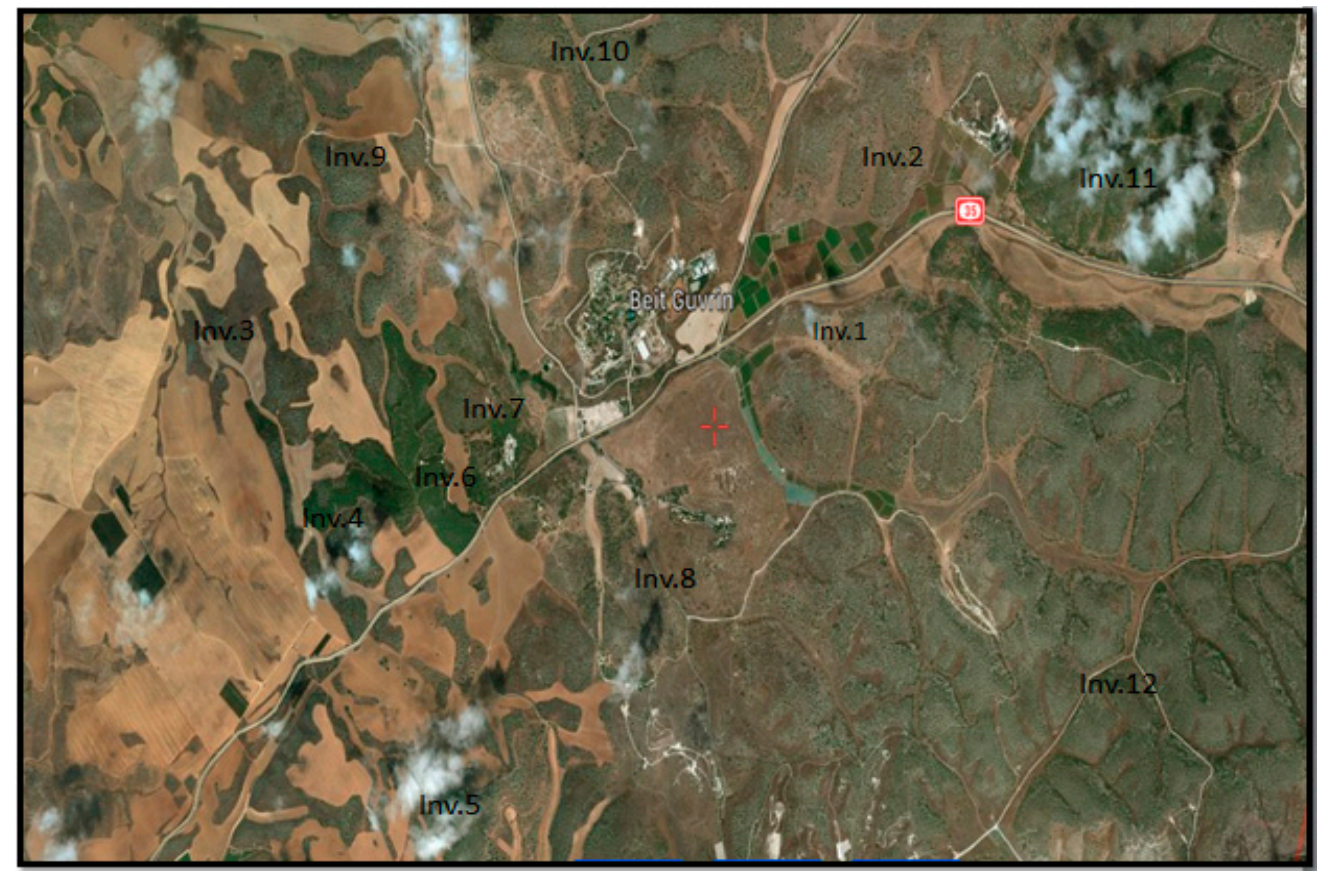

(B)

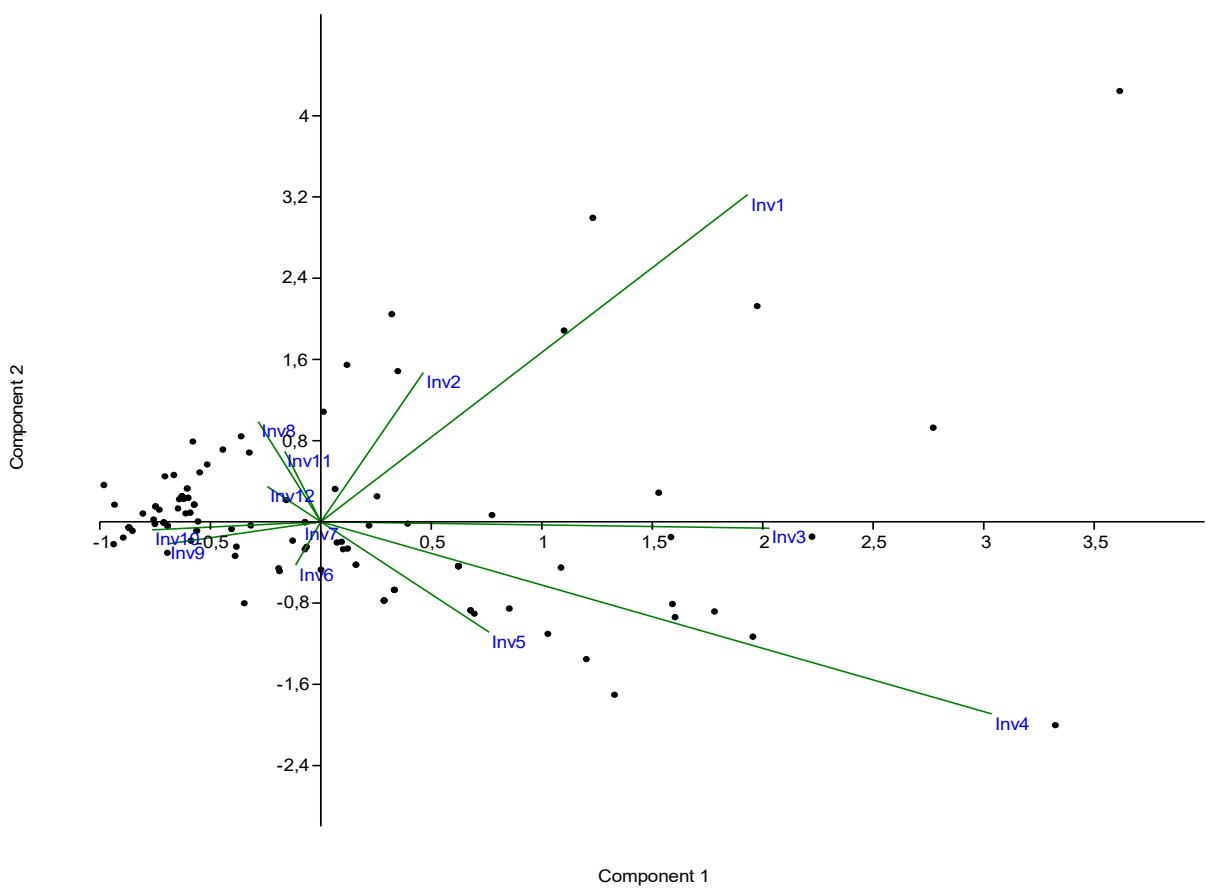

(C)

Figure 2. Cont. 


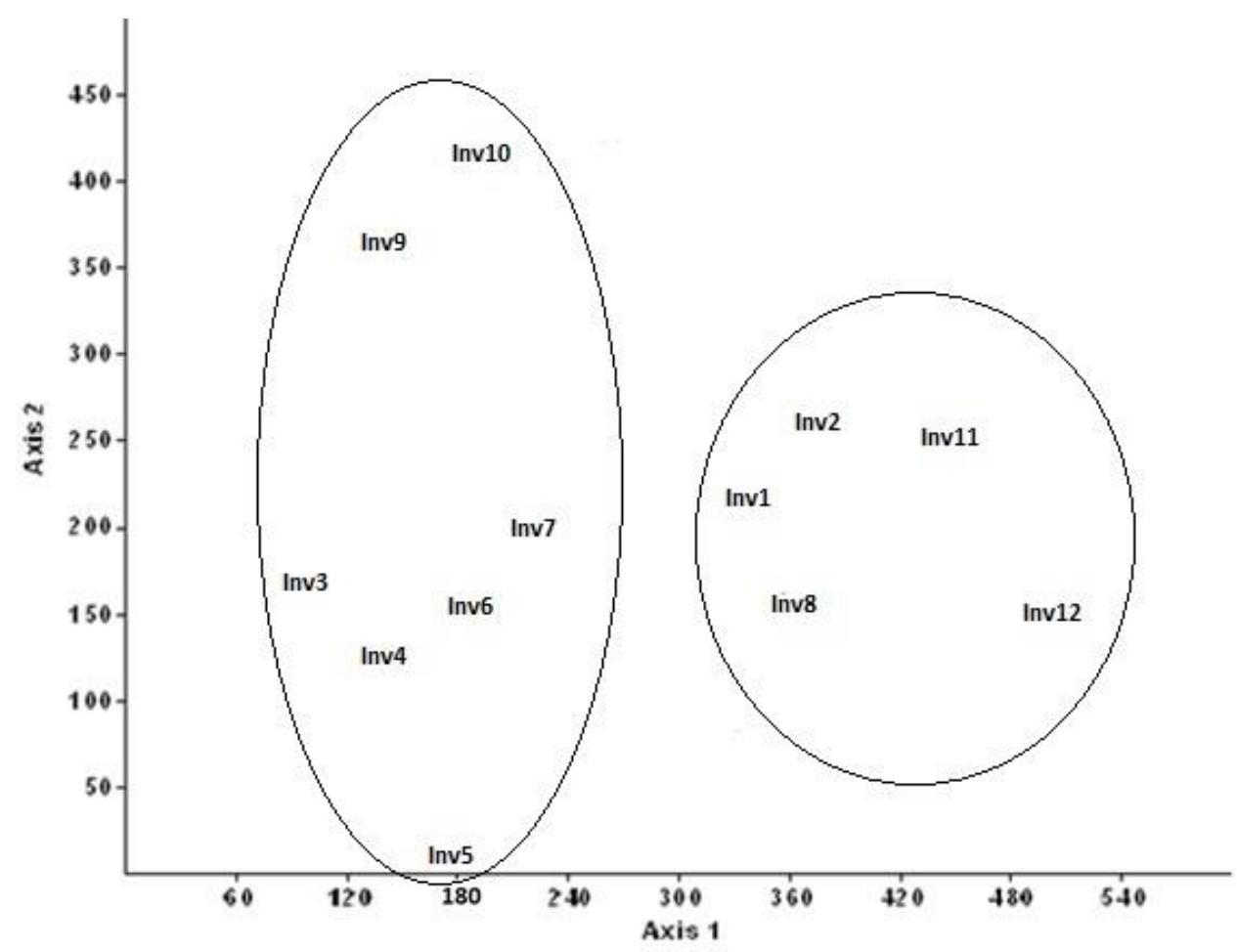

(D)

Figure 2. (A) The study area from which samples were taken is located in Beit Jibrin; (B) the study area and where the samples were selected by satellite; $(\mathbf{C})$ the principal component analysis; and (D) detrended correspondence analysis.

\subsection{Statistical Analyses}

Data were used to create an Excel table with 290 rows (plants) and 12 columns (relèves); from this table we created a Euclidean distance matrix (DCA), to measure distance, and similarity, by the procedure known as the full correlation method. We subsequently applied principal component analysis (PCA), having previously generated two matrices of correlation and covariance values, and detrended correspondence analysis (DCA) ordination analysis. The statistical software tool used was Community Analysis Package (CAP) 4.0, producing two clearly distinct inventories. However, we have two associations or communities: association 1 (ASL1), consisting of forest samples (groups 1, 2, 8, 11 and 12), and association 2 (ASL2), consisting of groups 3, 4, 5, 6, 7, 9 and 10, which were grouped together in (PCA) and (DCA).

\section{Result and Discussion}

\subsection{Forests Vegetation}

Cupresso sempervirentis-Pinetum halepensis ass. nova

Inventories 1, 2, 8, 11 and 12 were dominated by species belonging to Pinus and coniferous woodland, such as C. sempervirens L., C. arizonica L., C. macrocarpaa L., P. halepensis Miller, P. pinea L., P. canariensis C. Smith, P. brutia Tenore, J. phoenicea L., J. excelsa M. Bieb., and J. drupacea Labill., the association dominated by P. halepensis Miller, P. pinea L., P. canariensis C. Smith., P. brutia Tenore, C. sempervirens L., C. sempervirens L. var. horizontalis Miller, C. arizonica Greene, T. occidentalis L., J. phoenicea L., J. excelsa M. Bieb., J. drupacea Labill., A. monspessulanum L., F. retusa L., F. sycomorus L., F. cariaca L., C. equesitifolia L. M. alba L., M. nigara L., O. ficus indica (L.) Mill., O. robusta J.C. Wendl., O. ficus-barbarica A. Berger, S. alba L., P. alba L., P. nigra L., P. euphratica Oliver, Q. calliprinos Webb. or Q. palaestina K., Q. inthaburensis Decne., Q. boissieri Reut. or Q. boissieri Reut. var. latifolia (Boiss.) Zohary, Q. infectoria Olivier, Q. cerris L., etc., P. lentiscus L., R. palaestinus Boiss. 
(R. lycioides L.), R. alaternus L., Z. Spina-christi L., A. spinosa L., R. palaestinum Feinbrun, A. foetida L., C. abyssinica Kunth and Bouche, L. barbarum L., L. europaeum L., L. depressum Stocks, L. schweinfurthii Dammer, L. shawii Roem. and Schult., S. sinaicum Boiss., S. incanum L., P. pungens Willd., P. brachyodon (Boiss.) Zohary, P. chrysophylla Boiss., B. arabica (Boiss.) Maire and Weiller, P. platystegia Post., P. viscosa Poiret., S. dominica L., S. lanigera Poir., S. thymbra L., S. thymbrifolia Hedge and Feinbrun, S. palaestina L., S. fruticosa Miller, S. officinalis L., S. palaestina Benth., S. aethiopis L., M. fruticosa (L.) Druce., T. capitatum L., T. creticum L., T. capitata (L.) Cav., T. spicata L., B. populneus (Schott and Endl.) R.Br., C. spinosa L., C. sicula Duh., C. aegyptia Lam., A. halimus L., N. mucronata (Forssk.) Asch. and Schweinf., H. persicum Bunge, H.negevensis (Iljin and Zohary) L. Boulos, A. macrostachyum (Moric.) K. Koch, A. javanica (Burm.f.) Juss. ex Schult., S. fruticosa (L.) A. J. Scott, S. palaestina Eig. and Zohary, H. lancifolius (Boiss.) Kothe-Heinr., H. salicornicum (Moq.) Bunge ex Boiss., O. natrix L., L. nobilis L., P. aquilinum (L.) Kuhn, M. azedarach L., P. mascula (L.) Miller, A. filiculoides Lam., L. pyrotechnica (Forssk.) Decne., S. officinalis L., A. aleppica DC., G. tournefortii L., A. arborescens L., A. monosperma Delile, A. garcinii (Burm.f.) DC., P. dioscoridis (L.) DC., A. sieberi Besser., A. horridus L., A. palaestinus Baker, G. villosa Willd, E. aphylla Forskal, E. foeminea Forssk, A. halimus L., A. setifera Moq., A. syriaca Iljin, A. orientalis (L.) Boiss., A. strigosa Boiss. and Hohen., A. tinctoria (L.) Tausch, A. altissima (Miller) Swingle, R. chalepensis L., T. hirsuta (L.) Endl., V. eremobium Murb., V. fruticulosum Post., H. helix L., E. crassifolium L'Her., E. glaucophyllum (L.) L'Hér., E. arborescens (Desf.) Willd., E. acaule (L.) Becherer and Thell., E. creticum Lam., E. falcatum F. Delaroche, E. glomeratum Lam., E. maritimum L., E. cannabinum L., E. hierosolymitana Boiss., E. hirta L., E. hirsuta L., E. terracina L., $B$. aegyptiaca (L.) Delile, Z. dumosum Boiss., F. bruguieri DC., F. mollis Delile, F. orientalis C. Presl., F. arabica L., C. arabica (Boiss.) Diagn. Pl. Orient, C. lanatus Vahl., C. colocynthis (L.) Schrader, C.s dorycnium L., I. cairica (L.) Sweet, I. imperati (Vahl.) Griseb., H. aureus L., P. orientalis (L.) Feinbrun, M. myrtifolia Boiss. et Hohen., M. nervosa (Desf.) Benth., C. insulare (Candargy) Govaerts, E. cannabinum L., D. bovei (DC.) Anderb., H. sanguineum (L.) Kostel., I. maris-mortui Feinbrun, C. iphionoides (Boiss. and Blanche) Brul., C tinctoria (L.) J. Gay, C. reuteriana Boiss., C. syriaca Boiss., E. philistaeus Feinbrun and Zohary, V. vilosa Roth., F. thymifolia (L.) Webb., G. canum Req. ex DC., G. elongatum C. Presl., G. humifusum M. Bieb., C. acutum L., E. glomeratum Poir., E. fruticosum Desf., E. angustifolium Mill., P. orientalis (L.) Feinbrun, H. bacciferum Forssk., H. arbainense Fresen., M. ciliata (Forskal) I. M. Johnston, C. creticum Mill., F. vulgare Miller, F. biverticillata J. Thieb, F. communis L., F. orientalis L., F. tingitana L., F. syriaca Boiss., C. maculatum L., F. clypeata (L.) Medik, F. eriocarpa (DC.) Boiss., D. harra (Forssk.) Boiss., E. crassipes Fisch. and C. A. Mey., F. bisumbellata (Forssk.) Bubani, F. tenacissima L., V. cruciatum Sieber ex. Boiss., G. arabicum Fresen., G. flavum Crantz, G. grandiflorum Boiss. and A. Huet., H. micranthus L., H. hemistemon J. Gay, H. bulbosum L., F. arundinacea Schreb., H. triquetrifolium Turra, A. parvifolia Sm., I. palaestina (Baker) Boiss., J. unilateralis (Roem. and Schult.) O'Donell, K. aegyptiaca (L.) Nabelek, K. judaica Danin, L. nudicaulis (L.) Hooker fil., L. tuberosus L., L. bicolor (Boiss.) Eig. and Feinbrun, L. pyrotechnica (Forssk.) Decne., F. ferruginea (L.), V. tiberiadis Boiss., V. sinaiticum Benth., V. galilaeum Boiss., V. jordanicum Murb., V. gaillardotii Boiss., V. officinalis L. and V. luteola (Jacq.) Benth. species. Additionally, the community has 23 (7.84\%) endemic species, including the following endemic species: R. palaestinus Boiss., P. palaestina Boiss., A. ramonensis Danin, P. syriaca Boiss. and T. palaestina Bertol., accompanied by C. arizonica Greene and some Cupressus species. Forests grew in thermomediterranean-mesomediterranean thermotype regions and dry to humid environments in the soil of carbon substrates, such as brown ruinsenas and light rendzina (terra rossa), with an almost neutral $\mathrm{pH}$, and were habituated in the Mediterranean woodlands, shrub lands and relict maquis trees [16-19,52-62]. The slope was 10-30\%, with a plant cover rate of $70 \%$, an average altitude of $314 \mathrm{~m}$ and a vegetation height of 9-15 m (12 m) (Table 2). The distributions for life forms of this association are: 251 species, of which $96(38.09 \%)$ were phanerophytes trees (67), phanerophytes shrubs (15), phanerophytes shrub climbers (3), phanerophytes shrub vines (3), phanerophytes dwarf shrubs (3) and phanerophytes (5); 47 were shrubs, (18.65\%) as shrubs (28), chamaephyte semi-shrubs (18) 
and a chamaephyte shrub climber (1); 48 were chamaephytes (19.04\%), as chamaephytes (46), a chamaephyte parasite (1) and a chamaephyte-hemicryptophyte-annual (1); 51 were hemicryptophytes $(20.23 \%)$, as hemicryptophytes (51) and a hemicryptophyte climber (1); 5 were geophytes (1.98\%), as geophytes (4) and a geophyte vine (1); and 3 were helophytes $(1.19 \%)$ (Table 2).

Table 2. Cupresso sempervirentis-Pinetum halepensis ass. nova.

\begin{tabular}{|c|c|c|c|c|c|c|c|c|c|}
\hline Releve of Inventories & 1 & 2 & 8 & 11 & 12 & $\mathbf{P}$ & Status & Life Form & Family \\
\hline Surface in $\mathrm{m}^{2} 1=10$ & 450 & 600 & 500 & 550 & 400 & $\mathrm{R}$ & & & \\
\hline Cover rate $\%$ & 75 & 65 & 70 & 80 & 60 & $\mathrm{E}$ & & & \\
\hline Altitude in $\mathrm{m}$. & 325 & 285 & 300 & 310 & 350 & S & & & \\
\hline $\begin{array}{l}\text { Average height of } \\
\text { vegetation (m.) }\end{array}$ & 9 & 11 & 15 & 13 & 12 & $\mathrm{~N}$ & & & \\
\hline Slope \% & 25 & 30 & 10 & 15 & 15 & $\mathrm{C}$ & & & \\
\hline Orientation & $\mathrm{E}$ & $\mathrm{N}$ & $S$ & $\mathrm{~W}$ & $\mathrm{~N}$ & I & & & \\
\hline Order number & 1 & 2 & 8 & 11 & 12 & A & & & \\
\hline \multicolumn{10}{|c|}{ Characteristic of association and higher units } \\
\hline Pinus halepensis (L.) Miller & 5 & 4 & 2 & 2 & 1 & $\mathrm{~V}$ & $\mathrm{~N}$ & $\mathrm{~T}$ & Pinaceae \\
\hline Cupressus sempervirens L. & 4 & 4 & 2 & 2 & 2 & IV & $\mathrm{N}$ & $\mathrm{T}$ & Cupressaceae \\
\hline Pinus pinea $\mathrm{L}$. & 1 & 3 & & & 1 & Iv & $\mathrm{N}$ & $\mathrm{T}$ & Pinaceae \\
\hline Pinus canariensis C. Smith & & 1 & & 1 & & $\mathrm{I}$ & $\mathrm{N}$ & $\mathrm{T}$ & Pinaceae \\
\hline \multicolumn{10}{|l|}{ Companions } \\
\hline Cupressus arizonica Greene & 3 & 3 & & 2 & 1 & Iv & $\mathrm{N}$ & $\mathrm{T}$ & Pinaceae \\
\hline Thuja occidentalis L. & & 1 & & & & I & $\mathrm{N}$ & $\mathrm{T}$ & Cupressaceae \\
\hline Juniperus phoenicea L. & 1 & 1 & 1 & 1 & & II & $\mathrm{N}$ & $\mathrm{T}$ & Cupressaceae \\
\hline Juniperus excelsa M. Bieb. & & 1 & & 1 & & II & $\mathrm{N}$ & $\mathrm{T}$ & Cupressaceae \\
\hline Juniperus drupacea Labill. & 1 & & 2 & & 1 & II & $\mathrm{N}$ & Phan. shrub & Cupressaceae \\
\hline $\begin{array}{l}\text { Quercus calliprinos Webb. Quercus } \\
\text { palaestina } \mathrm{K} .\end{array}$ & 1 & 1 & & 2 & 1 & Iv & $\mathrm{N}$ & $\mathrm{T}$ & Fagaceae \\
\hline Quercus look Kotschy & & & & & & & $\mathrm{E}$ & $\mathrm{T}$ & Fagaceae \\
\hline Quercus inthaburensis Decne. & 3 & 2 & & & & II & $\mathrm{N}$ & $\mathrm{T}$ & Fagaceae \\
\hline Quercus libani G. Olivier & & & & & & & $\mathrm{N}$ & $\mathrm{T}$ & Fagaceae \\
\hline Quercus infectoria Olivier & & 1 & & 2 & & II & $\mathrm{N}$ & $\mathrm{T}$ & Fagaceae \\
\hline Quercus boissieri Reut. & & 1 & & & & $\mathrm{I}$ & $\mathrm{N}$ & $\mathrm{T}$ & Fagaceae \\
\hline Quercus cerris L. & 1 & & & & & I & $\mathrm{N}$ & $\mathrm{T}$ & Fagaceae \\
\hline Arbutus unedo L. & & & & & & & $\mathrm{N}$ & $\mathrm{T}$ & Ericaceae \\
\hline Pistacia lentiscus L. & 2 & 1 & 1 & & 1 & III & $\mathrm{N}$ & $\mathrm{T}$ & Anacardiaceae \\
\hline Pistacia palaestina Boiss. & & 1 & 1 & 1 & & II & $\mathrm{E}$ & $\mathrm{T}$ & Anacardiaceae \\
\hline Pistacia saportae Burnat. & 1 & 1 & & 1 & & II & $\mathrm{N}$ & $\mathrm{T}$ & Anacardiaceae \\
\hline Pistacia atlantica Desf. & 1 & 1 & & 1 & & II & $\mathrm{N}$ & $\mathrm{T}$ & Anacardiaceae \\
\hline Pistacia khinjuk Stocks & & 1 & & 1 & & I & $\mathrm{N}$ & $\mathrm{T}$ & Anacardiaceae \\
\hline Schinus molle L. & & 1 & & & & $\mathrm{I}$ & $\mathrm{N}$ & $\mathrm{T}$ & Anacardiaceae \\
\hline Rhus coriaria $\mathrm{L}$. & & & & & & & $\mathrm{N}$ & $\mathrm{T}$ & Anacardiaceae \\
\hline Schinus terebinthifolius Raddi & & 1 & & & & I & $\mathrm{N}$ & $\mathrm{T}$ & Anacardiaceae \\
\hline Rhus tripartita (Ucria) Grande & & & & & & & $\mathrm{N}$ & Phan. shrub & Anacardiaceae \\
\hline Rhamnus palaestinus Boiss. & & 1 & 2 & 2 & 2 & III & $\mathrm{E}$ & Phan. & Rhamnaceae \\
\hline Rhamnus disperma Ehrenb. e & $x$ Boiss & & 2 & 1 & & III & $\mathrm{N}$ & Phan. & Rhamnaceae \\
\hline Rhamnus alaternus L. & 1 & & 1 & 2 & 1 & III & $\mathrm{N}$ & $\mathrm{T}$ & Rhamnaceae \\
\hline Zizyphus Spina-christi L. I & esf. & & 1 & & & $\mathrm{I}$ & $\mathrm{N}$ & $\mathrm{T}$ & Rhamnaceae \\
\hline Zizyphus Lotus (L.) Lam. & & & & 1 & & I & $\mathrm{N}$ & Shrub & Rhamnaceae \\
\hline Paliurus spina-christi Miller & & & 1 & 1 & & $\mathrm{I}$ & $\mathrm{N}$ & Shrub & Rhamnaceae \\
\hline Ziziphus jujuba Miller & & 1 & & 1 & & $\mathrm{I}$ & $\mathrm{N}$ & Shrub & Rhamnaceae \\
\hline $\begin{array}{l}\text { Sageretia thea (Osbeck) } \\
\text { M. C. Johnst. }\end{array}$ & & & & 1 & & I & $\mathrm{N}$ & Phan. & Rhamnaceae \\
\hline Mespilus germanica L. & & & & 1 & & I & $\mathrm{N}$ & Shrub & Rosaceae \\
\hline Crataegus azarolus L. & & & & & 1 & $\mathrm{I}$ & $\mathrm{N}$ & $\mathrm{T}$ & Rosaceae \\
\hline Amygdalus ramonensis $\mathrm{D}$ & $\operatorname{nin}$ & & & 1 & & I & $\mathrm{E}$ & $\mathrm{T}$ & Rosaceae \\
\hline Prunus dulcis (Mill.) D. A. Web & & 2 & 1 & 1 & 1 & III & $\mathrm{N}$ & $\mathrm{T}$ & Rosaceae \\
\hline
\end{tabular}


Table 2. Cont.

\begin{tabular}{|c|c|c|c|c|c|c|c|c|}
\hline Releve of Inventories & 2 & 8 & 11 & 12 & $\mathbf{P}$ & Status & Life Form & Family \\
\hline Crataegus oriana (L.) DC & & 1 & & & I & $\mathrm{N}$ & Cham. & Rosaceae \\
\hline Sarcopoterium spinosum (L.) Spach & 1 & 1 & 1 & 1 & III & $\mathrm{N}$ & $\mathrm{T}$ & Rosaceae \\
\hline Pyrus syriaca Boiss. & & 1 & & & I & $\mathrm{E}$ & $\mathrm{T}$ & Rosaceae \\
\hline Crataegus monogyna Jacq. & & 1 & & & $\mathrm{I}$ & $\mathrm{N}$ & $\mathrm{T}$ & Rosaceae \\
\hline Malus communis Desf. & & & 1 & & I & $\mathrm{N}$ & Shrub & Rosaceae \\
\hline Pyracantha coccinea M. Roem. & & & 1 & & I & $\mathrm{N}$ & Shrub & Rosaceae \\
\hline Prunus ursina Kotschy & & 1 & 1 & & $\mathrm{I}$ & $\mathrm{N}$ & $\mathrm{T}$ & Rosaceae \\
\hline Prunus korshinskyi Hand. Mazz. & & & 1 & & $\mathrm{I}$ & $\mathrm{N}$ & Tree & Rosaceae \\
\hline Prunus arabica (Olivier) Meikle. & & & 1 & 1 & $\mathrm{I}$ & $\mathrm{N}$ & Phan. shrub & Rosaceae \\
\hline Rubus sanguineus Friv. & & & & & & $\mathrm{N}$ & Phan. shrub & Rosaceae \\
\hline Ceratonia siliqua L. & 1 & 1 & 2 & & III & $\mathrm{N}$ & $\mathrm{T}$ & Fabaceae \\
\hline Spartium junceum L. & 1 & 1 & & & I & $\mathrm{N}$ & Shrub & Fabaceae \\
\hline Cersis siliquastrum L. & 1 & 1 & & & I & $\mathrm{N}$ & $\mathrm{T}$ & Fabaceae \\
\hline Glycyrrhiza glabra L. & & & & & & $\mathrm{N}$ & Phan. shrub & Fabaceae \\
\hline Acacia salicina Lindl. & & 1 & & & I & $\mathrm{N}$ & $\mathrm{T}$ & Fabaceae \\
\hline Acacia cyanophylla Lindl. & & 1 & & & I & $\mathrm{N}$ & $\mathrm{T}$ & Fabaceae \\
\hline Calicotome villosa (Poir.) Link & & 1 & 1 & & $\mathrm{I}$ & $\mathrm{N}$ & $\mathrm{T}$ & Fabaceae \\
\hline Retama raetam (Forssk.) Webb. and Berthel. & 1 & & & & $\mathrm{I}$ & $\mathrm{N}$ & Phan. shrub & Fabaceae \\
\hline $\begin{array}{l}\text { Genista monspessulana (L.) } \\
\text { O. Bolós and Vigo. }\end{array}$ & 1 & & & & I & $\mathrm{N}$ & $\mathrm{T}$ & Fabaceae \\
\hline Acacia dealbata Link & 1 & & & & I & $\mathrm{N}$ & $\mathrm{T}$ & Fabaceae \\
\hline Acacia radiana Savi. & 1 & & & & $\mathrm{I}$ & $\mathrm{N}$ & $\mathrm{T}$ & Fabaceae \\
\hline Ficus microcarpa L.F. & & 1 & 1 & & I & $\mathrm{N}$ & $\mathrm{T}$ & Mimosaceae \\
\hline Ficus sycomorus L. & + & & & & $\mathrm{I}$ & $\mathrm{N}$ & $\mathrm{T}$ & Mimosaceae \\
\hline Ficus cariaca L. & 2 & 1 & 1 & & III & $\mathrm{N}$ & $\mathrm{T}$ & Mimosaceae \\
\hline Morus alba L. & & & 1 & & I & $\mathrm{N}$ & $\mathrm{T}$ & Mimosaceae \\
\hline Morus nigara L. & & 1 & 1 & & $\mathrm{I}$ & $\mathrm{N}$ & $\mathrm{T}$ & Mimosaceae \\
\hline Prosopis farcta (Banks et Sol.) & & & & & I & $\mathrm{N}$ & $\begin{array}{l}\text { Cham., } \\
\text { se-shrub }\end{array}$ & Mimosaceae \\
\hline Olea europaea L. & 1 & 1 & 1 & 1 & II & $\mathrm{N}$ & $\mathrm{T}$ & Oleaceae \\
\hline Phillyria media L. & & & 1 & & $\mathrm{I}$ & $\mathrm{N}$ & $\mathrm{T}$ & Oleaceae \\
\hline Olea oleaster Hoffmanns. and Link & & & 1 & & $\mathrm{I}$ & $\mathrm{N}$ & $\mathrm{T}$ & Oleaceae \\
\hline Olea europaea var. sylvestris & & & 1 & 1 & I & $\mathrm{N}$ & $\mathrm{T}$ & Oleaceae \\
\hline Salix alba $\mathrm{L}$. & & 1 & & & $\mathrm{I}$ & $\mathrm{N}$ & $\mathrm{T}$ & Salicaceae \\
\hline Populus alba L. & & 1 & 1 & & $\mathrm{I}$ & $\mathrm{N}$ & $\mathrm{T}$ & Salicaceae \\
\hline Populus euphratica Oliv. & & 1 & 1 & & $\mathrm{I}$ & $\mathrm{N}$ & $\mathrm{T}$ & Salicaceae \\
\hline Tamarix aphylla L. & & & 1 & & $\mathrm{I}$ & $\mathrm{N}$ & $\mathrm{T}$ & Tamaricaceae \\
\hline Tamarix jordanis Boiss. & & & & & & $\mathrm{E}$ & $\mathrm{T}$ & Tamaricaceae \\
\hline Tamarix palaestina Bertol. & & 1 & & & I & $\mathrm{E}$ & $\mathrm{T}$ & Tamaricaceae \\
\hline Tamarix nilotica (Ehrenb.) Bunge & & & & & & $\mathrm{N}$ & $\mathrm{T}$ & Tamaricaceae \\
\hline Tamarix negevensis Zohary & & & & & & $\mathrm{E}$ & $\mathrm{T}$ & Tamaricaceae \\
\hline Tamarix parviflora DC. & & & & & & $\mathrm{N}$ & $\mathrm{T}$ & Tamaricaceae \\
\hline Tamarix tetragyna Ehrenb. & 1 & & & & $\mathrm{I}$ & $\mathrm{N}$ & $\mathrm{T}$ & Tamaricaceae \\
\hline Tamarix gennessarensis Zohary & & & & & & $\mathrm{E}$ & $\mathrm{T}$ & Tamaricaceae \\
\hline $\begin{array}{l}\text { Reaumuria negevensis } \\
\text { Zohary and Danin }\end{array}$ & 1 & 1 & & & $\mathrm{I}$ & $\mathrm{E}$ & $\mathrm{T}$ & Tamaricaceae \\
\hline Tamarix passerinoides Delile & & & & & & $\mathrm{N}$ & $\mathrm{T}$ & Tamaricaceae \\
\hline Tamarix senegalensis DC. & & & & & & $\mathrm{N}$ & $\mathrm{T}$ & Tamaricaceae \\
\hline Tamarix amplexicaulis Ehrenb. & & & & & & $\mathrm{N}$ & $\mathrm{T}$ & Tamaricaceae \\
\hline Acer obtusifolium Sm. & & & & & & $\mathrm{E}$ & $\mathrm{T}$ & Aceracea \\
\hline Acer monspessulanum L. & & & 1 & & I & $\mathrm{N}$ & $\mathrm{T}$ & Aceracea \\
\hline Polygonum palaestinum Zohary & 1 & & 1 & & $\mathrm{I}$ & $\mathrm{E}$ & Hem. & Polygonaceae \\
\hline Persicaria lanigera (R.Br.) Sojak & & & & & & $\mathrm{N}$ & Hem. & Polygonaceae \\
\hline Atraphaxis spinosa L. & & & 1 & & & $\mathrm{~N}$ & Cham. & Polygonaceae \\
\hline Rheum palaestinum Feinbrun & 1 & & & 1 & II & $\mathrm{E}$ & Hem. & Polygonaceae \\
\hline Anagyris foetida L. & & & & & & $\mathrm{N}$ & Phan. shrub & $\begin{array}{c}\text { Papilionaceae/ } \\
\text { Leguminosae }\end{array}$ \\
\hline
\end{tabular}


Table 2. Cont.

\begin{tabular}{|c|c|c|c|c|c|c|c|c|c|}
\hline Releve of Inventories & 1 & 2 & 8 & 11 & 12 & $\mathbf{P}$ & Status & Life Form & Family \\
\hline \multicolumn{3}{|c|}{ Colutea abyssinica Kunth and Bouche } & & & & & $\mathrm{N}$ & Phan. shrub & $\begin{array}{l}\text { Papilionaceae/ } \\
\text { Leguminosae }\end{array}$ \\
\hline \multicolumn{3}{|l|}{ Lycium barbarum L. } & 1 & 1 & & I & $\mathrm{N}$ & Shrub & Solanaceae \\
\hline \multicolumn{3}{|l|}{ Lycium europaeum L. } & & 1 & & $\mathrm{I}$ & $\mathrm{N}$ & Shrub & Solanaceae \\
\hline \multicolumn{3}{|c|}{ Nicotiana glauca Graham. } & & & & & $\mathrm{N}$ & $\mathrm{T}$ & Solanaceae \\
\hline \multicolumn{3}{|c|}{ Lycium depressum Stocks } & 1 & & & $\mathrm{I}$ & $\mathrm{N}$ & Phan. shrub & Solanaceae \\
\hline \multicolumn{3}{|c|}{ Lycium schweinfurthii Dammer } & & 1 & 1 & $\mathrm{I}$ & $\mathrm{N}$ & Phan. shrub & Solanaceae \\
\hline \multicolumn{3}{|c|}{ Lycium shawii Roem. and Schult. } & & 1 & 1 & $\mathrm{I}$ & $\mathrm{N}$ & Shrub & Solanaceae \\
\hline \multicolumn{2}{|c|}{ Solanum sinaicum Boiss. } & & 1 & 1 & & $\mathrm{I}$ & $\mathrm{N}$ & $\begin{array}{l}\text { Cham., } \\
\text { s-shrub }\end{array}$ & Solanaceae \\
\hline \multicolumn{2}{|l|}{ Solanum incanum L. } & & 1 & 1 & & I & $\mathrm{N}$ & $\begin{array}{l}\text { Cham., } \\
\text { s-shrub }\end{array}$ & Solanaceae \\
\hline Phlomis pungens Willd. & 1 & & & 1 & & I & $\mathrm{N}$ & $\begin{array}{l}\text { Cham., } \\
\text { s-shrub }\end{array}$ & Lamiaceae \\
\hline \multicolumn{2}{|c|}{ Phlomis brachyodon (Boiss.) Zohary } & & 1 & 1 & & I & $\mathrm{N}$ & $\begin{array}{l}\text { Cham., } \\
\text { s-shrub }\end{array}$ & Lamiaceae \\
\hline \multicolumn{2}{|c|}{ Phlomis chrysophylla Boiss. } & & & & 1 & I & $\mathrm{N}$ & $\begin{array}{l}\text { Cham., } \\
\text { s-shrub }\end{array}$ & Lamiaceae \\
\hline \multirow{2}{*}{\multicolumn{2}{|c|}{$\begin{array}{l}\text { Ballota saxatilis Sieber ex. C. Presl } \\
\text { Ballota philistaea Bornm. }\end{array}$}} & & & & & & $\mathrm{N}$ & Shrub & Lamiaceae \\
\hline & & & 1 & & 1 & I & $\mathrm{E}$ & Shrub & Lamiaceae \\
\hline \multicolumn{4}{|c|}{ Ballota undulata (Sieber ex Fresen.) Bentham } & & & & $\mathrm{N}$ & Shrub & Lamiaceae \\
\hline \multicolumn{4}{|c|}{ Bassia arabica (Boiss.) Maire and Weiller } & 1 & & $\mathrm{I}$ & $\mathrm{N}$ & Shrub & Lamiaceae \\
\hline \multicolumn{2}{|c|}{ Phlomis platystegia Post. } & & & & & & $\mathrm{E}$ & $\begin{array}{l}\text { Cham., } \\
\text { s-shrub }\end{array}$ & Lamiaceae \\
\hline Phlomis viscosa Poiret. & 1 & & & & & I & $\mathrm{N}$ & $\begin{array}{l}\text { Cham., } \\
\text { s-shrub }\end{array}$ & Lamiaceae \\
\hline \multicolumn{2}{|l|}{ Salvia eigii Zohay } & & & & & & $\mathrm{E}$ & Hem., cham. & Lamiaceae \\
\hline \multicolumn{2}{|l|}{ Salvia dominica L. } & 1 & 1 & 1 & & II & $\mathrm{N}$ & $\begin{array}{l}\text { Cham., } \\
\text { s-shrub }\end{array}$ & Lamiaceae \\
\hline \multicolumn{2}{|l|}{ Salvia lanigera Poir. } & 1 & 1 & 1 & & II & $\mathrm{N}$ & $\begin{array}{l}\text { Cham., } \\
\text { s-shrub }\end{array}$ & Lamiaceae \\
\hline Satureja thymbra L. & 1 & 1 & 1 & 1 & & III & $\mathrm{E}$ & $\begin{array}{l}\text { Cham., } \\
\text { s-shrub }\end{array}$ & Lamiaceae \\
\hline \multicolumn{2}{|c|}{ Satureja thymbrifolia Hedge and Feinbrun } & 1 & 1 & 1 & & II & $\mathrm{E}$ & $\begin{array}{l}\text { Cham., } \\
\text { s-shrub }\end{array}$ & Lamiaceae \\
\hline Stachys palaestina L. & & 1 & 1 & 1 & & II & $\mathrm{E}$ & $\begin{array}{l}\text { Cham., } \\
\text { s-shrub }\end{array}$ & Lamiaceae \\
\hline Salvia fruticosa Mill. & 1 & 1 & 1 & & & II & $\mathrm{N}$ & Cham. & Lamiaceae \\
\hline Salvia officinalis L. & 1 & 1 & 1 & 1 & & III & $\mathrm{N}$ & Cham. & Lamiaceae \\
\hline Salvia aegyptiaca L. & & 1 & 1 & & & II & $\mathrm{N}$ & Cham. & Lamiaceae \\
\hline Salvia palaestina Benth. & 1 & & 1 & 1 & & II & $\mathrm{E}$ & Cham. & Lamiaceae \\
\hline Salvia aethiopis L. & & & & & & & $\mathrm{N}$ & Cham. & Lamiaceae \\
\hline Micromeria fruticosa & ace. & & 1 & 1 & & II & $\mathrm{N}$ & Cham. & Lamiaceae \\
\hline Teucrium capitatum L. & 1 & 2 & 1 & 1 & & III & $\mathrm{N}$ & Cham. & Lamiaceae \\
\hline Teucrium creticum L. & 2 & 1 & 1 & 1 & & III & $\mathrm{N}$ & Cham. & Lamiaceae \\
\hline Thymbra capitata (L.) & & 1 & 1 & & & I & $\mathrm{N}$ & Cham. & Lamiaceae \\
\hline Thymbra spicata L. & & & 1 & 1 & & $\mathrm{I}$ & $\mathrm{N}$ & Cham. & Lamiaceae \\
\hline $\begin{array}{l}\text { Brachychiton populn } \\
\text { (Schott and Endl.) R }\end{array}$ & & 1 & & & & I & $\mathrm{N}$ & $\mathrm{T}$ & Malvaceae \\
\hline Jacaranda mimosaefc & & & & & & & $\mathrm{N}$ & $\mathrm{T}$ & Bignoniaceae \\
\hline Capparis spinosa $\mathrm{L}$. & & 1 & & & & $\mathrm{I}$ & $\mathrm{N}$ & Shrub & Capparaceae \\
\hline Capparis sicula Duh. & & & 1 & & & I & $\mathrm{N}$ & Shrub & Capparaceae \\
\hline Capparis aegyptia Lam. & & & 1 & & & $\mathrm{I}$ & $\mathrm{N}$ & Shrub & Capparaceae \\
\hline Celtis australis L. & & & & & & & $\mathrm{N}$ & $\mathrm{T}$ & Ulmaceae \\
\hline Casuarina equesitifoli & & & & & & & $\mathrm{N}$ & $\mathrm{T}$ & Casuarenaceae \\
\hline Atriplex halimus L. & & & 1 & & & I & $\mathrm{N}$ & Shrub & Amaranthaceae \\
\hline $\begin{array}{l}\text { Noaea mucronata (For } \\
\text { Asch. and Schwein }\end{array}$ & & 1 & 1 & & & I & $\mathrm{N}$ & Shrub & Amaranthaceae \\
\hline
\end{tabular}


Table 2. Cont.

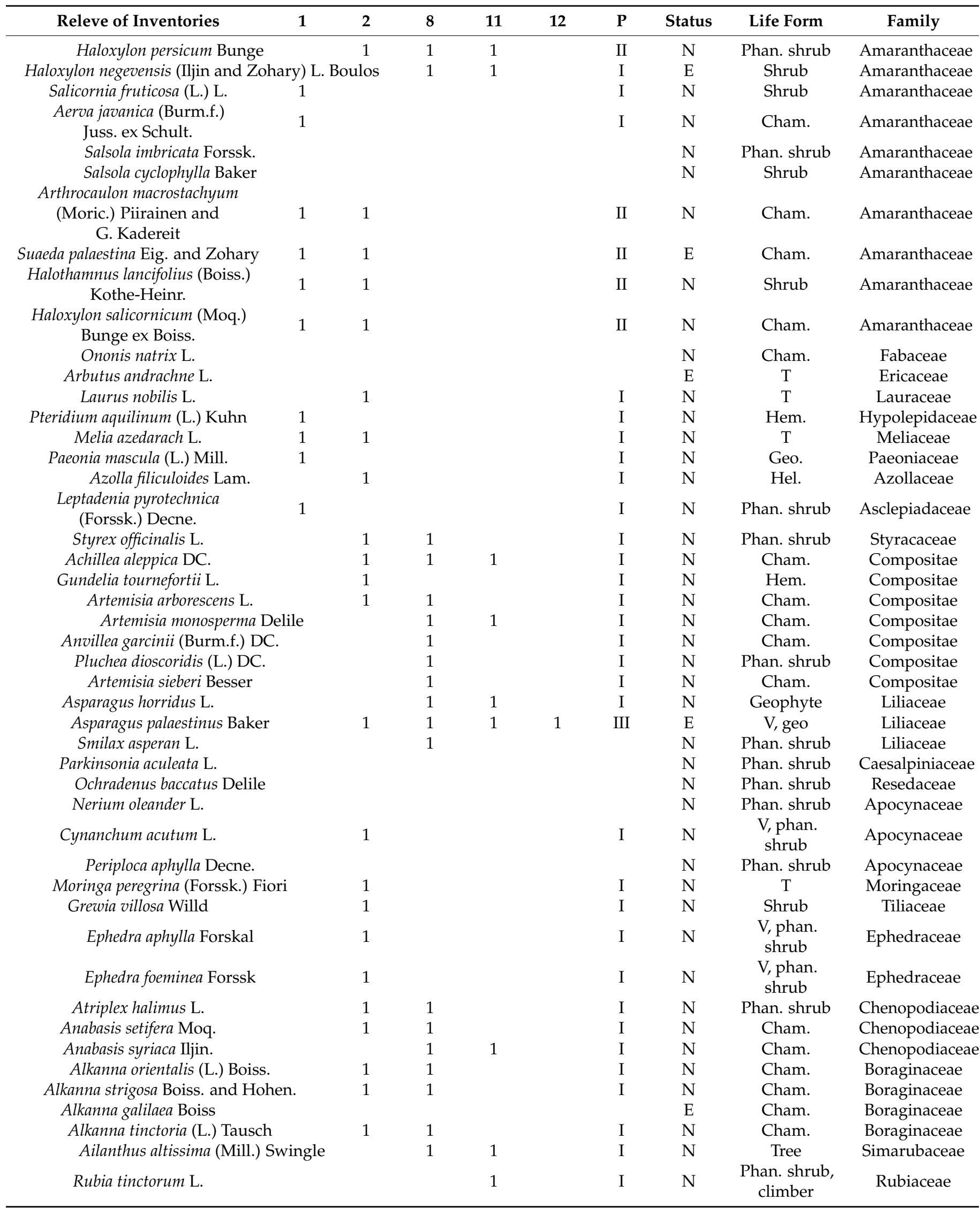


Table 2. Cont.

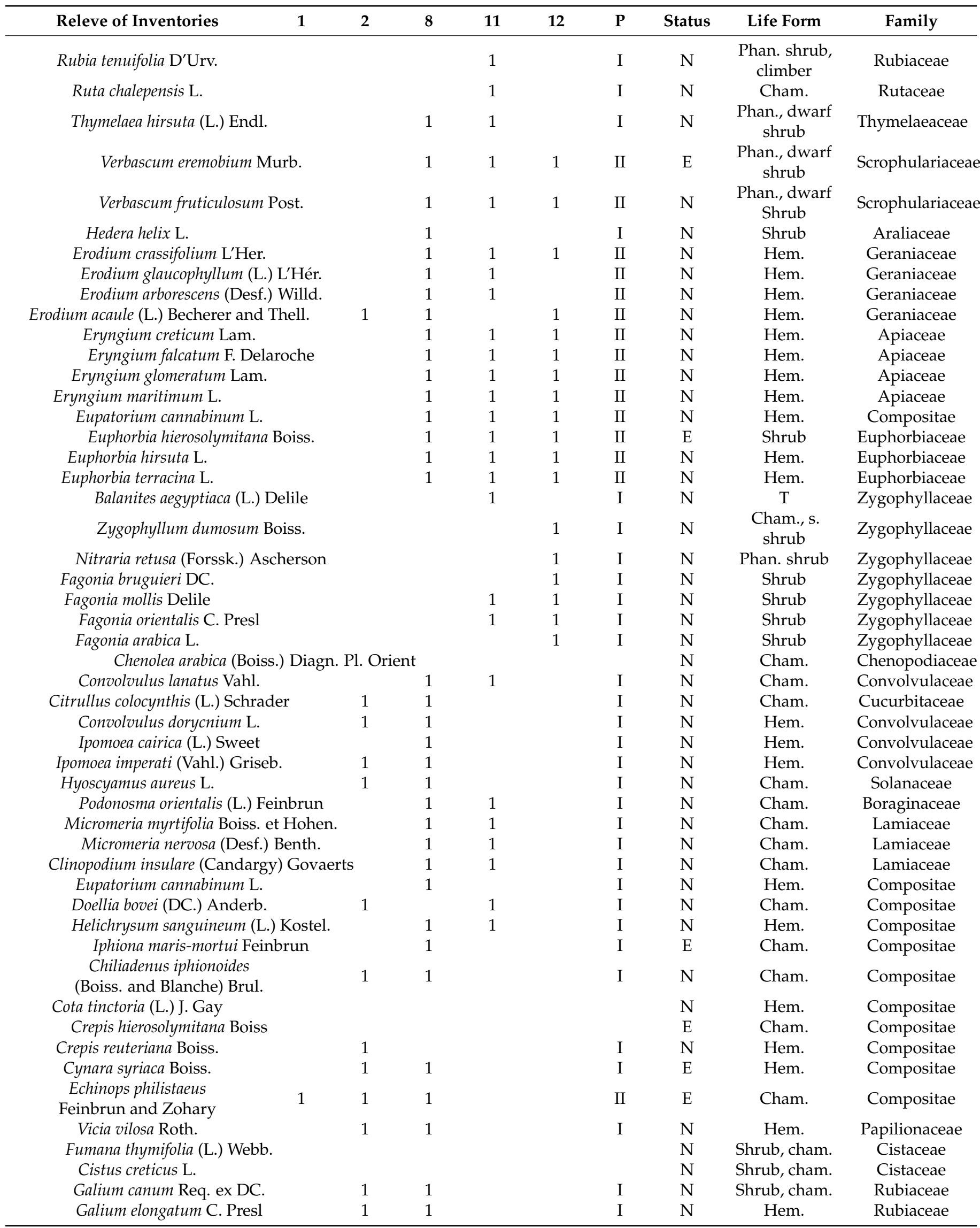


Table 2. Cont.

\begin{tabular}{|c|c|c|c|c|c|c|c|c|c|}
\hline Releve of Inventories & 1 & 2 & 8 & 11 & 12 & $\mathbf{P}$ & Status & Life Form & Family \\
\hline \multicolumn{2}{|l|}{ Galium humifusum M. Bieb. } & 1 & 1 & & & $\mathrm{I}$ & $\mathrm{N}$ & Hem. & Rubiaceae \\
\hline \multicolumn{2}{|l|}{ Cynanchum acutum L. } & 1 & & 1 & & $\mathrm{I}$ & $\mathrm{N}$ & $\begin{array}{l}\text { Phan. shrub, } \\
\text { climber }\end{array}$ & Apocynaceae \\
\hline \multicolumn{2}{|l|}{ Echium glomeratum Poir. } & 1 & & 2 & & $\mathrm{I}$ & $\mathrm{N}$ & Hem. & Boraginaceae \\
\hline \multicolumn{2}{|l|}{ Echiochilon fruticosum Desf. } & & & 1 & & I & $\mathrm{N}$ & Cham. & Boraginaceae \\
\hline \multicolumn{2}{|l|}{ Echium angustifolium Mill. } & & & 1 & & $\mathrm{I}$ & $\mathrm{N}$ & Cham. & Boraginaceae \\
\hline \multicolumn{2}{|c|}{ Heliotropium maris-mortui Zohary } & & & & & & $\mathrm{E}$ & Shrub, cham. & Boraginaceae \\
\hline \multicolumn{2}{|c|}{ Podonosma orientalis (L.) Feinbrun } & & & 1 & & $\mathrm{I}$ & $\mathrm{N}$ & Cham. & Boraginaceae \\
\hline \multicolumn{2}{|c|}{ Heliotropium bacciferum Forssk. } & & 1 & 1 & & $\mathrm{I}$ & $\mathrm{N}$ & Sh, cham. & Boraginaceae \\
\hline \multicolumn{2}{|c|}{ Heliotropium arbainense Fresen. } & & & & 1 & $\mathrm{I}$ & $\mathrm{N}$ & Shrub, cham. & Boraginaceae \\
\hline \multicolumn{2}{|l|}{ Moltkiopsis ciliata (Forskal) I. M. Johnston } & 1 & 1 & & & $\mathrm{I}$ & $\mathrm{N}$ & Cham. & Boraginaceae \\
\hline \multicolumn{2}{|l|}{ Heliotropium rotundifolium Lehm } & 1 & 1 & & & $\mathrm{I}$ & $\mathrm{N}$ & Cham. & Boraginaceae \\
\hline \multicolumn{2}{|l|}{ Cynoglossum creticum Mill. } & & 1 & & & $\mathrm{I}$ & $\mathrm{N}$ & Cham. & Boraginaceae \\
\hline \multicolumn{2}{|l|}{ Morettia canescens Boiss } & & & & & & $\mathrm{N}$ & Cham. & Brassicaceae \\
\hline \multicolumn{6}{|c|}{ Najas marina var. Intermedia (Wolfg. ex Gorski) Rendle } & & $\mathrm{N}$ & Helophyte & Hydrocharitaceae \\
\hline \multicolumn{6}{|c|}{ Deverra triradiata } & & $\mathrm{N}$ & Cham. & Apiaceae \\
\hline \multicolumn{2}{|l|}{ Foeniculum vulgare Miller } & & 1 & & & $\mathrm{I}$ & $\mathrm{N}$ & Hem. & Apiaceae \\
\hline \multicolumn{2}{|l|}{ Ferula biverticillata J. Thieb. } & 1 & & & & $\mathrm{I}$ & $\mathrm{N}$ & Hem. & Apiaceae \\
\hline Ferula communis $\mathrm{L}$. & 1 & & & & & $\mathrm{I}$ & $\mathrm{N}$ & Hem. & Apiaceae \\
\hline Ferula orientalis L. & 1 & & & & & I & $\mathrm{E}$ & Hem. & Apiaceae \\
\hline Ferula tingitana L. & 1 & & & & & $\mathrm{I}$ & $\mathrm{N}$ & Hem. & Apiaceae \\
\hline Ferulago syriaca Boiss. & 1 & & & & & $\mathrm{I}$ & $\mathrm{N}$ & Hem. & Apiaceae \\
\hline Conium maculatum L. & & 1 & & & & $\mathrm{I}$ & $\mathrm{N}$ & Hem. & Apiaceae \\
\hline Fibigia clypeata (L.) Medik & & 1 & & & & $\mathrm{I}$ & $\mathrm{N}$ & Hem. & Brassicaceae \\
\hline Fibigia eriocarpa (DC.) Boiss. & & & 1 & & & $\mathrm{I}$ & $\mathrm{N}$ & Hem. & Brassicaceae \\
\hline Diplotaxis harra (Forssk.) Boiss. & & 1 & & & & $\mathrm{I}$ & $\mathrm{N}$ & $\begin{array}{l}\text { Cham., hem., } \\
\text { annual }\end{array}$ & Brassicaceae \\
\hline Erysimum crassipes Fisch. and C. & A. $\mathrm{M}$ & & 1 & & & $\mathrm{I}$ & $\mathrm{N}$ & Hem. & Brassicaceae \\
\hline $\begin{array}{l}\text { Fimbristylis bisumbellata } \\
\text { (Forssk.) Bubani }\end{array}$ & 1 & & & & & $\mathrm{I}$ & $\mathrm{N}$ & Helophyte & Cyperaceae \\
\hline Forsskaolea tenacissima L. & & 1 & & & & $\mathrm{I}$ & $\mathrm{N}$ & Cham., Hem. & Urticaceae \\
\hline Viscum cruciatum Sieber and Bioss & & 1 & & & & $\mathrm{I}$ & $\mathrm{N}$ & $\begin{array}{l}\text { Cham., } \\
\text { parasite }\end{array}$ & Santalaceae \\
\hline Glaucium arabicum Fresen. & & 1 & & & & $\mathrm{I}$ & $\mathrm{N}$ & Hem. & Papaveraceae \\
\hline Glaucium flavum Crantz & & 1 & & & & $\mathrm{I}$ & $\mathrm{N}$ & Hem. & Papaveraceae \\
\hline $\begin{array}{l}\text { Glaucium grandiflorum } \\
\text { Boiss. and A. Huet }\end{array}$ & 1 & & & & & $\mathrm{I}$ & $\mathrm{N}$ & Hem. & Papaveraceae \\
\hline Vitex agnus-castus L. & & & & & & $\mathrm{I}$ & $\mathrm{N}$ & $\begin{array}{l}\text { Cham., } \\
\text { s-shrub }\end{array}$ & Verbenaceae \\
\hline Globularia arabica Jaub. and S & pach & & & & & $\mathrm{I}$ & $\mathrm{N}$ & Cham., shrub & Plantaginaceae \\
\hline Hibiscus micranthus L. & & & & & & $\mathrm{I}$ & $\mathrm{N}$ & Cham., shrub & Malvaceae \\
\hline Herniaria hemistemon J. Gay & & 1 & & & & $\mathrm{I}$ & $\mathrm{N}$ & Hem. & Caryophyllaceae \\
\hline Hordeum bulbosum L. & & 1 & 1 & & & $\mathrm{I}$ & $\mathrm{N}$ & Hem. & Poaceae \\
\hline Festuca arundinacea Schreb & & 1 & 1 & & & $\mathrm{I}$ & $\mathrm{N}$ & Hem. & Poaceae \\
\hline Hypericum triquetrifolium Turra & & 1 & 1 & & & $\mathrm{I}$ & $\mathrm{N}$ & Hem. & Hypericaceae \\
\hline Aristolochia parvifolia $\mathrm{Sm}$. & & & 1 & & & $\mathrm{I}$ & $\mathrm{N}$ & Hem. climber & Aristolochiaceae \\
\hline Iris atrofusca Baker & & & & & & & $\mathrm{E}$ & Geo. & Iridaceae \\
\hline Iris atropurpurea Baker & & & & & & & $\mathrm{E}$ & Geo. & Iridaceae \\
\hline Iris palaestina (Baker) Boiss. & & 1 & & & & $\mathrm{I}$ & $\mathrm{E}$ & Geo. & Iridaceae \\
\hline Iris vartanii Foster & & & & & & & $\mathrm{E}$ & Geo. & Iridaceae \\
\hline Gladiolus italicus Mill. & & & & & & & $\mathrm{N}$ & Geo. & Iridaceae \\
\hline Juncus acutus L. & & & & & & & $\mathrm{N}$ & Hem. & Juncaceae \\
\hline Juncus articulates L. & & & & & & & $\mathrm{N}$ & Hem. & Juncaceae \\
\hline Juncus subulatus Forssk. & & & & & & & $\mathrm{N}$ & Hem. & Juncaceae \\
\hline $\begin{array}{l}\text { Jacquemontia unilateralis (Roem. } \\
\text { and Schult.) O'Donell }\end{array}$ & 1 & & & & & $\mathrm{I}$ & $\mathrm{N}$ & Cham. & Convolvulaceae \\
\hline Kickxia aegyptiaca (L.) Nabelek & & 1 & & & & $\mathrm{I}$ & $\mathrm{N}$ & Cham. & Plantaginaceae \\
\hline
\end{tabular}


Table 2. Cont.

\begin{tabular}{|c|c|c|c|c|c|c|c|c|}
\hline Releve of Inventories & 2 & 8 & 11 & 12 & $\mathbf{P}$ & Status & Life Form & Family \\
\hline Kickxia judaica Danin & & 1 & & & I & $\mathrm{N}$ & Cham. & Plantaginaceae \\
\hline Launaea nudicaulis (L.) Hooker fil. & & 1 & & & I & $\mathrm{N}$ & Hem. & Compositae \\
\hline Leontodon tuberosus L. & 1 & & & & $\mathrm{I}$ & $\mathrm{N}$ & Hem. & Compositae \\
\hline Leopoldia bicolor (Boiss.) Eig. and Feinbrun & & 1 & & & I & $\mathrm{N}$ & Geo. & Asparagaceae \\
\hline Leptadenia pyrotechnica (Forssk.) Decne. & & 1 & & & I & $\mathrm{N}$ & Phan. & Apocynaceae \\
\hline Fimbristylis ferruginea (L.) $\quad 1$ & & & & & I & $\mathrm{N}$ & Helophyte & Cyperaceae \\
\hline Verbascum tiberiadis Boiss. & 1 & 1 & 1 & & II & $\mathrm{N}$ & Hem. & Scrophulariaceae \\
\hline Verbascum sinaiticum Benth. & 1 & 1 & 1 & & II & $\mathrm{N}$ & Hem. & Scrophulariaceae \\
\hline Verbascum galilaeum Boiss. & 1 & 1 & & 1 & II & $\mathrm{N}$ & Hem. & Scrophulariaceae \\
\hline Verbascumjordanicum Murb. & 1 & 1 & & 1 & II & $\mathrm{N}$ & Hem. & Scrophulariaceae \\
\hline Verbascum gaillardotii Boiss. & 1 & 1 & & 1 & II & $\mathrm{N}$ & Hem. & Scrophulariaceae \\
\hline Verbena officinalis L. & 1 & 1 & 1 & & II & $\mathrm{N}$ & Hem. & Verbenaceae \\
\hline Vigna luteola (Jacq.) Benth. & & & & 1 & $\mathrm{I}$ & $\mathrm{N}$ & $\begin{array}{l}\text { Cham., s-sh., } \\
\text { climber }\end{array}$ & Papilionaceae \\
\hline Clematis flammula L. & & & & & & $\mathrm{N}$ & $\mathrm{V}$, phan. & Anunculaceae \\
\hline Clematis cirrhosa L. & & & & & & $\mathrm{N}$ & $\mathrm{V}$, phan. & Anunculaceae \\
\hline
\end{tabular}

Abbreviations: T, tree; Phan shrub, phanerophyte shrubs; Phan. shrub climber, phanerophyte shrub climber; Phan. shrub vine, phanerophyte shrub vine; Phan. vine, phanerophyte vine; Phan. dwarf shrubs, phanerophyte dwarf shrubs; Cham., chamaephyte; Cham. s.-shrubs, chamaephyte semi-shrubs; Sh, cham., chamaephyte shrublet; Cham., Shrub, chamaephyte shrubs; Cham. shrub climber, chamaephyte shrub climber; Cham.hem., chamaephyte-hemicryptophyte; Cham., p., chamaephyte parasite; Cham., hem., annual, chamaephytehemicryptophyte-annual; Hem., hemicryptophyte; Hem. climber, hemicryptophyte climber; Geo., geophyte; Geo. vine, geophyte vine; and Hel., helophyte.

These give us an indication that suggests that association one lies in the Asian regions of the Eastern Mediterranean, and thermomediterranean to mesomedoterranean thermotypes: Cupresso sempervirentis - Pinetum halepensis ass. nova. (Figure 2C; Table 2: ASL 1- Inv. $1,2,8,11$ and 12, typus inv. 1).

Percentage of plant species present in the sample studies and communities: $\mathrm{V}=100 \%$, IV $=60.1-80 \%$, III $=40.1-60 \%$, II $=20.1-40 \%$ and I $=0.1-20 \%$. N: native, E: endemic, Sh: shrub, ASL: association and ASL2: association 2.

\subsection{Forest Maquis, Macchie and Steppe Vegetation \\ 3.2.1. Pistacio lentisci-Quercetum calliprini ass. nova}

The second association consisted of the forest group (inventories 3, 4, 6, 7, 9, 10 and 5), represented by Quercus genus as Q. calliprinos Webb. (Q. palaestina K., Oak Palestine) [63,64], Q. inthaburensis Decne., Q. infectoria Olivier, Q. boissieri Reut. and Q. cerris L. Additionally, in May 2018 several new varieties of oak were identified, including Q. suber L., Q. ilex L. and Q. robur L., in addition to common oak species, when the geographic information system (GIS) platform technology was used in various areas of occupied Palestine in 1948 by Ezra Barnea [65]. Rhamnus and Pistachio genus, as species of P. lentiscus L., R. palaestinus Boiss. (R. lycioides L.), R. alaternus L., Z. Spina-christi L. Desf. and Z. Lotus (L.) Lam., are heavy forest plains with 37 endemic plants (12.75\%), including: R. palaestinus Boiss., P. palaestina Boiss, Q. look Kotschy, A. andrachne L., B. philistaea Bornm., A. obtusifolium Sm., T. palaestina Bertol. and A. ramonensis Danin, accompanied by R. palaestinus Boiss., C. siliqua L. and others Quercus species. The forest or community is in steppe environments that are part of a large area of uneven flat grassland in Southeast Europe and the western Mediterranean, with dry and semi-arid areas and an inframediterranean to thermomediterranean thermotype [16-19]. The slope is 5-25\%, with an average vegetation height of $8.5 \mathrm{~m}$, an average altitude of $272.8 \mathrm{~m}$ and a soil type of limestone and terra rosa. The distributions of life forms for this association are: $113(38.96 \%)$ phanerophytes (trees), 109 (37.58\%) shrubs and chamaephytes, 55 (17.93\%) hemicryptophytes, 9 (2.94\%) geophytes and $4(1.30 \%)$ helophytes. This suggested to us that the association is Pistacio lentisci-Quercetum calliprini ass. nova. hoc loco. (Figure 2C,D; Table 3: ASL 2- Inv. 3, 4, 6, 7, 9, 10 and 5). 
Table 3. Association 2. Pistacio lentisci-Quercetum calliprini ass. nova.

\begin{tabular}{|c|c|c|c|c|c|c|c|c|c|c|c|}
\hline Releve of Inventories & 3 & 4 & 5 & 6 & 7 & 9 & 10 & $\mathbf{P}$ & Status & Life Form & Family \\
\hline Surface in $\mathrm{m}^{2} 1=10$ & 390 & 350 & 320 & 550 & 400 & 300 & 300 & $\mathrm{R}$ & & & \\
\hline Cover rate \% & 75 & 70 & 85 & 80 & 60 & 75 & 80 & $\mathrm{E}$ & & & \\
\hline Altitude in $\mathbf{m}$. & 275 & 270 & 260 & 310 & 350 & 370 & 300 & $\mathrm{~S}$ & & & \\
\hline Average height of vegetation (m.) & 6 & 5.5 & 5 & 13 & 12 & 10 & 8 & $\mathrm{~N}$ & & & \\
\hline Slope $\%$ & 25 & 20 & 15 & 10 & 5 & 10 & 20 & $\mathrm{C}$ & & & \\
\hline Orientation & $\mathrm{N}$ & $\mathrm{E}$ & $\mathrm{N}$ & $\mathrm{E}$ & W & $\mathrm{E}$ & $\mathrm{W}$ & I & & & \\
\hline Order number & 3 & 4 & 5 & 6 & 7 & 9 & 10 & A & & & \\
\hline \multicolumn{12}{|c|}{ Characteristic of association and higher units } \\
\hline Pistacia lentiscus L. & 4 & 2 & 2 & 3 & 3 & 3 & 2 & $\mathrm{~V}$ & $\mathrm{~N}$ & $\mathrm{~T}$ & Anacardiaceae \\
\hline $\begin{array}{c}\text { Quercus calliprinos Webb. } \\
\text { (Quercus Palaestina K.) }\end{array}$ & 5 & 3 & 4 & 3 & 3 & 3 & 2 & $\mathrm{~V}$ & $\mathrm{~N}$ & $\mathrm{~T}$ & Fagaceae \\
\hline Quercus inthaburensis Decne. & 3 & 2 & & 1 & 1 & & 2 & IV & $\mathrm{N}$ & $\mathrm{T}$ & Fagaceae \\
\hline Quercus infectoria Olivier & 3 & 3 & & 1 & & 2 & 2 & IV & $\mathrm{N}$ & $\mathrm{T}$ & Fagaceae \\
\hline Pistacia palaestina Boiss. & 1 & & 1 & 2 & & 2 & 2 & IV & $\mathrm{E}$ & $\mathrm{T}$ & Anacardiaceae \\
\hline \multicolumn{12}{|l|}{ Companions } \\
\hline Rhamnus palaestinus Boiss. & 4 & 3 & & 3 & & 2 & 2 & IV & $\mathrm{E}$ & Phan. & Rhamnaceae \\
\hline \multicolumn{2}{|c|}{ Rhamnus disperma Ehrenb.ex Boiss. } & & 2 & & 2 & & 2 & III & $\mathrm{N}$ & Phan. & Rhamnaceae \\
\hline \multicolumn{2}{|l|}{ Rhamnus alaternus $\mathrm{L}$. } & 2 & 2 & & 2 & & 2 & III & $\mathrm{N}$ & $\mathrm{T}$ & Rhamnaceae \\
\hline Ceratonia siliqua $\mathrm{L}$ & 3 & 2 & 2 & & 2 & 2 & 2 & IV & $\mathrm{N}$ & $\mathrm{T}$ & Fabaceae \\
\hline Quercus look Kotschy & & 1 & & & & & & $\mathrm{I}$ & $\mathrm{E}$ & $\mathrm{T}$ & Fagaceae \\
\hline Quercus libani G. Olivier & & 1 & & & & & & $\mathrm{I}$ & $\mathrm{N}$ & $\mathrm{T}$ & Fagaceae \\
\hline Quercus boissieri Reut. & & 1 & & & & & & $\mathrm{I}$ & $\mathrm{N}$ & $\mathrm{T}$ & Fagaceae \\
\hline Quercus cerris L. & 1 & & & & & & & I & $\mathrm{N}$ & $\mathrm{T}$ & Fagaceae \\
\hline Pistacia atlantica Desf. & 1 & 2 & & 2 & & 2 & & III & $\mathrm{N}$ & $\mathrm{T}$ & Anacardiaceae \\
\hline Pistacia khinjuk Stocks & & 1 & & & & & & $\mathrm{I}$ & $\mathrm{N}$ & $\mathrm{T}$ & Anacardiaceae \\
\hline Schinus molle L. & & 1 & & & & & & I & $\mathrm{N}$ & $\mathrm{T}$ & Anacardiaceae \\
\hline Rhus coriaria $\mathrm{L}$. & & & & & & & & $\mathrm{I}$ & $\mathrm{N}$ & $\mathrm{T}$ & Anacardiaceae \\
\hline Schinus terebinthifolius Raddi & & 1 & & & & & & I & $\mathrm{N}$ & $\mathrm{T}$ & Anacardiaceae \\
\hline Rhus tripartita (Ucria) Grande & & & & & & 1 & & $\mathrm{I}$ & $\mathrm{N}$ & Phan. shrub & Anacardiaceae \\
\hline Zizyphus Spina-christi L. D & & & & & & 1 & & $\mathrm{I}$ & $\mathrm{N}$ & $\mathrm{T}$ & Rhamnaceae \\
\hline Zizyphus Lotus (L.) Lam. & & & & & & 1 & & $\mathrm{I}$ & $\mathrm{N}$ & Shrub & Rhamnaceae \\
\hline Paliurus spina-christi Miller & & & & & & 1 & & I & $\mathrm{N}$ & Shrub & Rhamnaceae \\
\hline \multirow{2}{*}{\multicolumn{2}{|c|}{$\begin{array}{l}\text { Ziziphus jujuba Miller } \\
\text { Searsia tripartita (Ucria) Moffett }\end{array}$}} & & & 2 & & & & $\mathrm{I}$ & $\mathrm{N}$ & Shrub & Rhamnaceae \\
\hline & & & & & 1 & & & I & $\mathrm{N}$ & Phan. & Rhamnaceae \\
\hline \multicolumn{2}{|l|}{ Sageretia thea (Osbeck) } & & & & & & & I & $\mathrm{N}$ & Phan. & Rhamnaceae \\
\hline \multicolumn{2}{|l|}{ Arbutus unedo L. } & & & 1 & & & & I & $\mathrm{N}$ & $\mathrm{T}$ & Ericaceae \\
\hline \multicolumn{2}{|l|}{ Mespilus germanica L. } & & & & & & & $\mathrm{I}$ & $\mathrm{N}$ & Shrub & Rosaceae \\
\hline \multicolumn{2}{|l|}{ Crataegus azarolus L. } & & & 1 & & & 1 & $\mathrm{I}$ & $\mathrm{N}$ & $\mathrm{T}$ & Rosaceae \\
\hline \multicolumn{2}{|l|}{ Amygdalus ramonensis Danin } & & 2 & 2 & & 2 & 2 & III & $\mathrm{E}$ & $\mathrm{T}$ & Rosaceae \\
\hline \multicolumn{2}{|c|}{ Prunus dulcis (Mill.) D. A. Webb. } & & 2 & 2 & 2 & 2 & 2 & III & $\mathrm{N}$ & $\mathrm{T}$ & Rosaceae \\
\hline \multicolumn{2}{|c|}{ Crataegus oriana (L.) DC } & & & & 1 & 1 & & $\mathrm{I}$ & $\mathrm{N}$ & $\mathrm{T}$ & Rosaceae \\
\hline \multicolumn{2}{|c|}{ Sarcopoterium spinosum (L.) Spach } & & 2 & 2 & 2 & 2 & & III & $\mathrm{N}$ & Cham. & Rosaceae \\
\hline \multicolumn{2}{|l|}{ Pyrus syriac Boiss. } & & & & 1 & 1 & & $\mathrm{I}$ & $\mathrm{E}$ & $\mathrm{T}$ & Rosaceae \\
\hline Crataegus monogyna Jacq. & & & & & 1 & 1 & & $\mathrm{I}$ & $\mathrm{N}$ & $\mathrm{T}$ & Rosaceae \\
\hline Malus communis Desf. & & & & 1 & & 1 & & $\mathrm{I}$ & $\mathrm{N}$ & Shrub & Rosaceae \\
\hline Pyracantha coccinea $\mathrm{M}$. Roe & & & & & 1 & & & $\mathrm{I}$ & $\mathrm{N}$ & Shrub & Rosaceae \\
\hline Prunus ursina Kotschy & & & & 1 & & & & $\mathrm{I}$ & $\mathrm{N}$ & $\mathrm{T}$ & Rosaceae \\
\hline Prunus korshinskyi Hand. M & $\mathrm{zz}$. & & & & & 1 & & $\mathrm{I}$ & $\mathrm{N}$ & Tree & Rosaceae \\
\hline Prunus arabica (Olivier) Me & de. & & & & & 1 & & $\mathrm{I}$ & $\mathrm{N}$ & Phan. shrub & Rosaceae \\
\hline Rubus sanguineus Friv. & & & & & & 1 & & $\mathrm{I}$ & $\mathrm{N}$ & Phan. shrub & Rosaceae \\
\hline Pinus halepensis (L.) Miller & & & 1 & & 1 & & & $\mathrm{I}$ & $\mathrm{N}$ & $\mathrm{T}$ & Pinaceae \\
\hline Cupressus sempervirens L. & & & & & 2 & & & $\mathrm{I}$ & $\mathrm{N}$ & $\mathrm{T}$ & Cupressaceae \\
\hline Pinus Pinea L. & 1 & & & & 1 & & & I & $\mathrm{N}$ & $\mathrm{T}$ & Pinaceae \\
\hline Pinus canariensis C. Smith & & 1 & & 1 & & & & $\mathrm{I}$ & $\mathrm{N}$ & $\mathrm{T}$ & Pinaceae \\
\hline Pinus brutia Tenore & 1 & 1 & & 1 & & & & $\mathrm{I}$ & $\mathrm{N}$ & $\mathrm{T}$ & Cupressaceae \\
\hline Cupressus arizonica Greene & & & & & 1 & & & $\mathrm{I}$ & $\mathrm{N}$ & $\mathrm{T}$ & Pinaceae \\
\hline
\end{tabular}


Table 3. Cont.

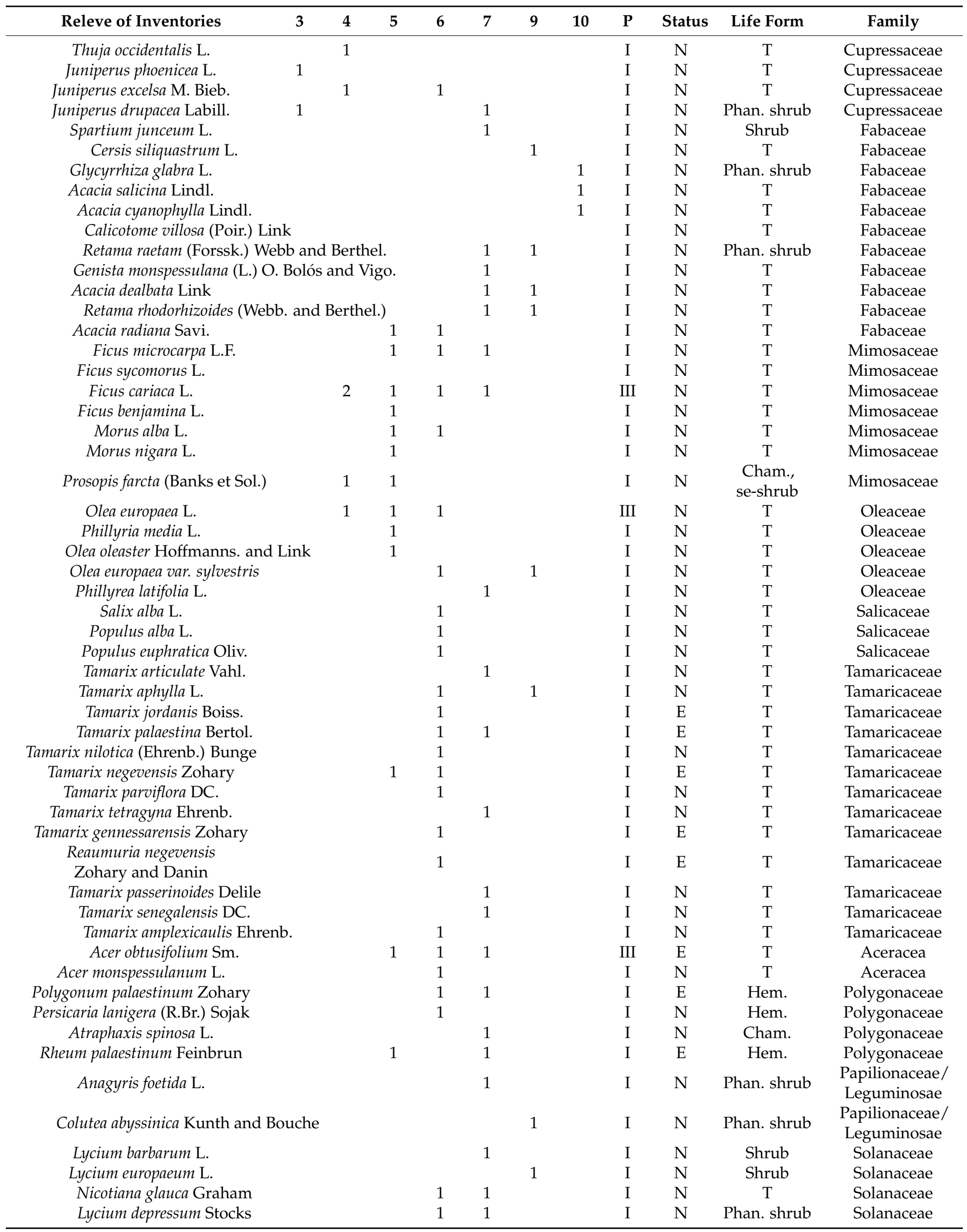


Table 3. Cont.

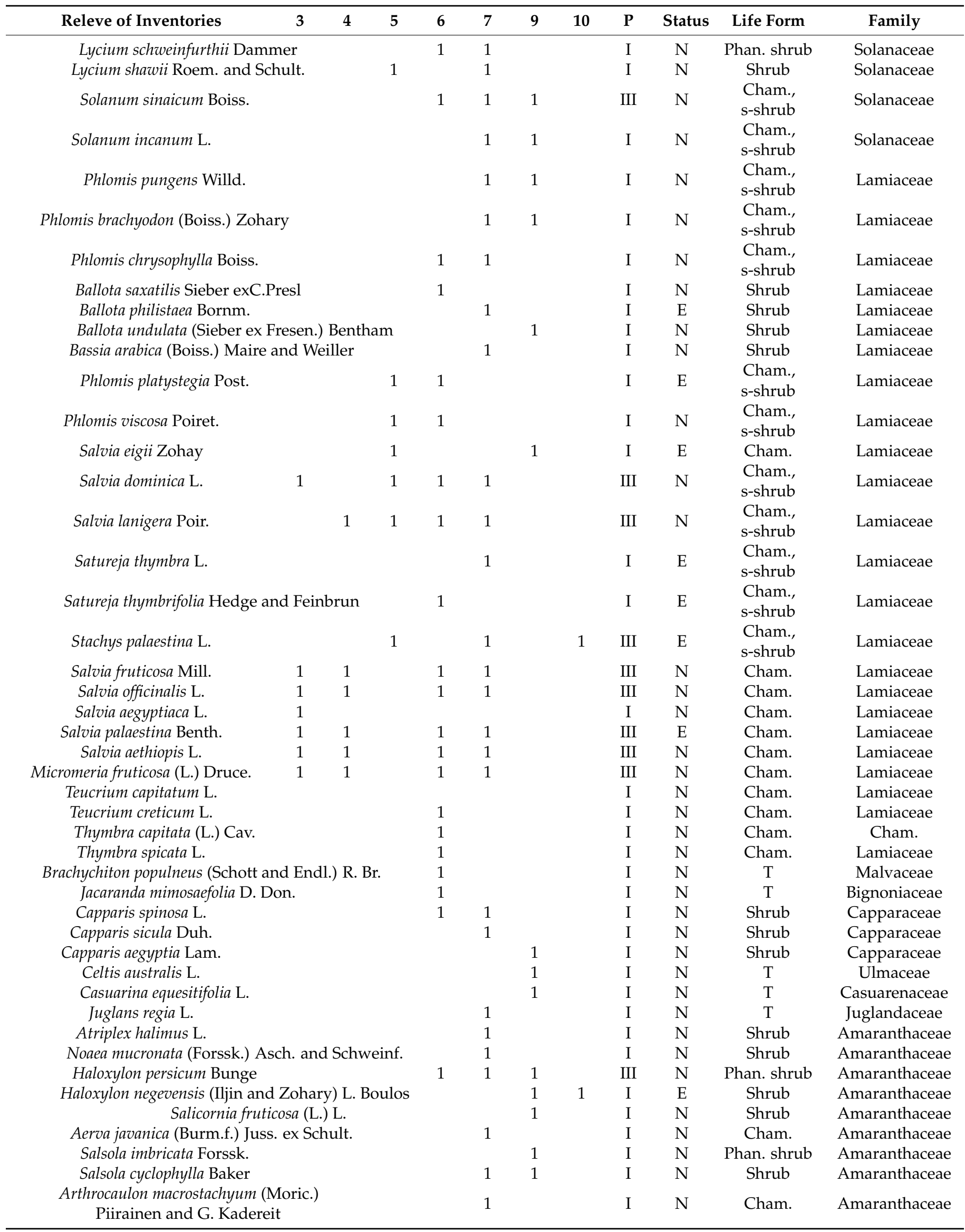


Table 3. Cont.

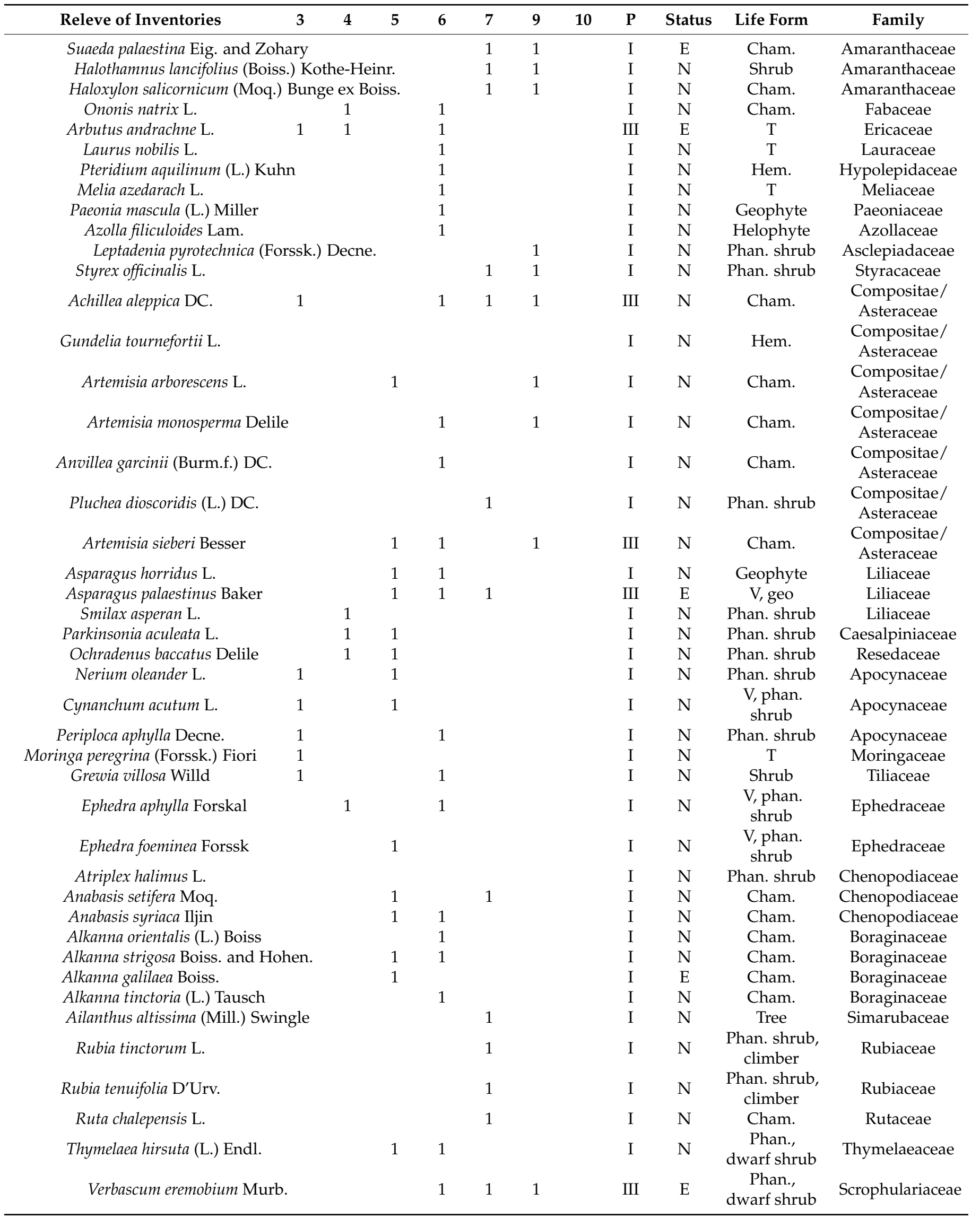


Table 3. Cont.

\begin{tabular}{|c|c|c|c|c|c|c|c|c|c|c|}
\hline Releve of Inventories & 3 & 5 & 6 & 7 & 9 & 10 & $\mathbf{P}$ & Status & Life Form & Family \\
\hline \multicolumn{3}{|c|}{ Verbascum fruticulosum Post. } & 1 & 1 & 1 & & III & $\mathrm{N}$ & $\begin{array}{c}\text { Phan., } \\
\text { dwarf shrub }\end{array}$ & Scrophulariaceae \\
\hline \multicolumn{3}{|l|}{ Hedera helix L. } & 1 & 1 & & & $\mathrm{I}$ & $\mathrm{N}$ & Shrub & Araliaceae \\
\hline \multicolumn{3}{|c|}{ Erodium crassifolium L'Her. } & & 1 & & & $\mathrm{I}$ & $\mathrm{N}$ & Hem. & Geraniaceae \\
\hline \multicolumn{3}{|c|}{ Erodium glaucophyllum (L.) L'Hér. } & & 1 & & & $\mathrm{I}$ & $\mathrm{N}$ & Hem. & Geraniaceae \\
\hline \multicolumn{3}{|c|}{ Erodium arborescens (Desf.) Willd. } & 1 & 1 & 1 & & III & $\mathrm{N}$ & Hem. & Geraniaceae \\
\hline \multicolumn{3}{|c|}{ Erodium acaule (L.) Becherer and Thell. } & 1 & 1 & & & I & $\mathrm{N}$ & Hem. & Geraniaceae \\
\hline \multicolumn{3}{|c|}{ Eryngium creticum Lam. } & 1 & & & & I & $\mathrm{N}$ & Hem. & Apiaceae \\
\hline \multicolumn{3}{|c|}{ Eryngium falcatum F. Delaroche } & 1 & & & & $\mathrm{I}$ & $\mathrm{N}$ & Hem. & Apiaceae \\
\hline \multicolumn{3}{|c|}{ Eryngium glomeratum Lam. } & 1 & & & & I & $\mathrm{N}$ & Hem. & Apiaceae \\
\hline \multicolumn{3}{|c|}{ Eryngium maritimum L. } & & 1 & & & $\mathrm{I}$ & $\mathrm{N}$ & Hem. & Apiaceae \\
\hline \multicolumn{3}{|c|}{ Eupatorium cannabinum L. } & & 1 & & & $\mathrm{I}$ & $\mathrm{N}$ & Hem. & Compositae \\
\hline \multicolumn{3}{|c|}{ Euphorbia hierosolymitana Boiss. } & 1 & 1 & & & $\mathrm{I}$ & $\mathrm{E}$ & Shrub & Euphorbiaceae \\
\hline \multicolumn{2}{|c|}{ Euphorbia hirta L. } & 1 & 1 & & & & I & $\mathrm{N}$ & Shrub & Euphorbiaceae \\
\hline \multicolumn{2}{|l|}{ Euphorbia hirsuta L. } & 1 & 1 & & & & $\mathrm{I}$ & $\mathrm{N}$ & Hem. & Euphorbiaceae \\
\hline \multicolumn{2}{|l|}{ Euphorbia terracina $\mathrm{L}$. } & & 1 & 1 & & & $\mathrm{I}$ & $\mathrm{N}$ & Hem. & Euphorbiaceae \\
\hline \multicolumn{2}{|c|}{ Balanites aegyptiaca (L.) Delile } & & 1 & 1 & & & $\mathrm{I}$ & $\mathrm{N}$ & $\mathrm{T}$ & Zygophyllaceae \\
\hline \multicolumn{2}{|c|}{ Zygophyllum dumosum Boiss. } & & 1 & & & & I & $\mathrm{N}$ & $\begin{array}{l}\text { Cham., } \\
\text { s.-shrub }\end{array}$ & Zygophyllaceae \\
\hline \multicolumn{2}{|c|}{ Nitraria retusa (Forssk.) Ascherson } & & 1 & & & & $\mathrm{I}$ & $\mathrm{N}$ & Phan. shrub & Zygophyllaceae \\
\hline \multicolumn{2}{|c|}{ Fagonia bruguieri DC. } & & 1 & & & & $\mathrm{I}$ & $\mathrm{N}$ & Shrub & Zygophyllaceae \\
\hline \multicolumn{3}{|l|}{ Fagonia mollis Delile } & & 1 & & & $\mathrm{I}$ & $\mathrm{N}$ & Shrub & Zygophyllaceae \\
\hline \multicolumn{3}{|c|}{ Fagonia orientalis C. Presl } & 1 & 1 & & & I & $\mathrm{N}$ & Shrub & Zygophyllaceae \\
\hline Fagonia arabica $\mathrm{L}$. & & & & 1 & & & $\mathrm{I}$ & $\mathrm{N}$ & Shrub & Zygophyllaceae \\
\hline Chenolea arabica (Boi & gn. Pl. Or & & & 1 & & & $\mathrm{I}$ & $\mathrm{N}$ & Cham. & Chenopodiaceae \\
\hline Convolvulus lanatus $\mathrm{V}$ & & & & 1 & & & $\mathrm{I}$ & $\mathrm{N}$ & Cham. & Convolvulaceae \\
\hline Citrullus colocynthis (L. & der & & & 1 & & & $\mathrm{I}$ & $\mathrm{N}$ & Cham. & Cucurbitaceae \\
\hline Convolvulus dorycniun & & & & 1 & & & $\mathrm{I}$ & $\mathrm{N}$ & Hem & Convolvulaceae \\
\hline Ipomoea cairica (L.) Sw & & & & 1 & & & $\mathrm{I}$ & $\mathrm{N}$ & Hem & Convolvulaceae \\
\hline Ipomoea imperati (Vah & & & & 1 & & & $\mathrm{I}$ & & Hem & Convolvulaceae \\
\hline Hyoscyamus aureus L. & & & & 1 & & & $\mathrm{I}$ & $\mathrm{N}$ & Cham. & Solanaceae \\
\hline Podonosma orientalis (L. & run & & & 1 & & & $\mathrm{I}$ & $\mathrm{N}$ & Cham. & Boraginaceae \\
\hline Micromeria myrtifolia & t Hohen. & & 1 & 1 & 1 & & III & $\mathrm{N}$ & Cham. & Lamiaceae \\
\hline Micromeria nervosa (De & & & 1 & 1 & 1 & & III & $\mathrm{N}$ & Cham. & Lamiaceae \\
\hline Clinopodium insulare & irgy) Gov & & & & & & I & $\mathrm{N}$ & Cham. & Lamiaceae \\
\hline Eupatorium cannabinur & & & 1 & & & & I & $\mathrm{N}$ & Hem. & $\begin{array}{l}\text { Compositae/ } \\
\text { Asteraceae }\end{array}$ \\
\hline Doellia bovei (DC.) And & & & & 1 & & & I & $\mathrm{N}$ & Cham. & $\begin{array}{l}\text { Compositae/ } \\
\text { Asteraceae }\end{array}$ \\
\hline Helichrysum sanguint & Kostel. & & 1 & 1 & & & I & $\mathrm{N}$ & Hem. & $\begin{array}{l}\text { Compositae/ } \\
\text { Asteraceae }\end{array}$ \\
\hline Iphiona maris-mortui & & & 1 & 1 & & & I & $\mathrm{E}$ & Cham. & $\begin{array}{l}\text { Compositae/ } \\
\text { Asteraceae }\end{array}$ \\
\hline Chiliadenus iphionoides ( & nd Blanch & Brul. & & & & & I & $\mathrm{N}$ & Cham. & $\begin{array}{l}\text { Compositae/ } \\
\text { Asteraceae }\end{array}$ \\
\hline Cota tinctoria (L.) J. Gay & & & 1 & 1 & & & I & $\mathrm{N}$ & Hem. & $\begin{array}{l}\text { Compositae/ } \\
\text { Asteraceae }\end{array}$ \\
\hline Crepis hierosolymitana $\mathrm{B}$ & & & 1 & & & & I & $\mathrm{N} / \mathrm{E}$ & Cham. & $\begin{array}{l}\text { Compositae/ } \\
\text { Asteraceae }\end{array}$ \\
\hline Crepis reuteriana Boiss. & & & 1 & & & & I & $\mathrm{N}$ & Hem. & $\begin{array}{l}\text { Compositae/ } \\
\text { Asteraceae }\end{array}$ \\
\hline Cynara syriaca Boiss. & & & 1 & 1 & & & I & $\mathrm{E}$ & Hem. & $\begin{array}{l}\text { Compositae/ } \\
\text { Asteraceae }\end{array}$ \\
\hline Echinops philistaeus Feir & nd Zohary & & 1 & 1 & 1 & & III & $\mathrm{E}$ & Cham. & $\begin{array}{l}\text { Compositae/ } \\
\text { Asteraceae }\end{array}$ \\
\hline Vicia vilosa Roth. & 1 & & & & & & I & $\mathrm{N}$ & Hem. & $\begin{array}{l}\text { Leguminosae/ } \\
\text { Papilionaceae }\end{array}$ \\
\hline
\end{tabular}


Table 3. Cont.

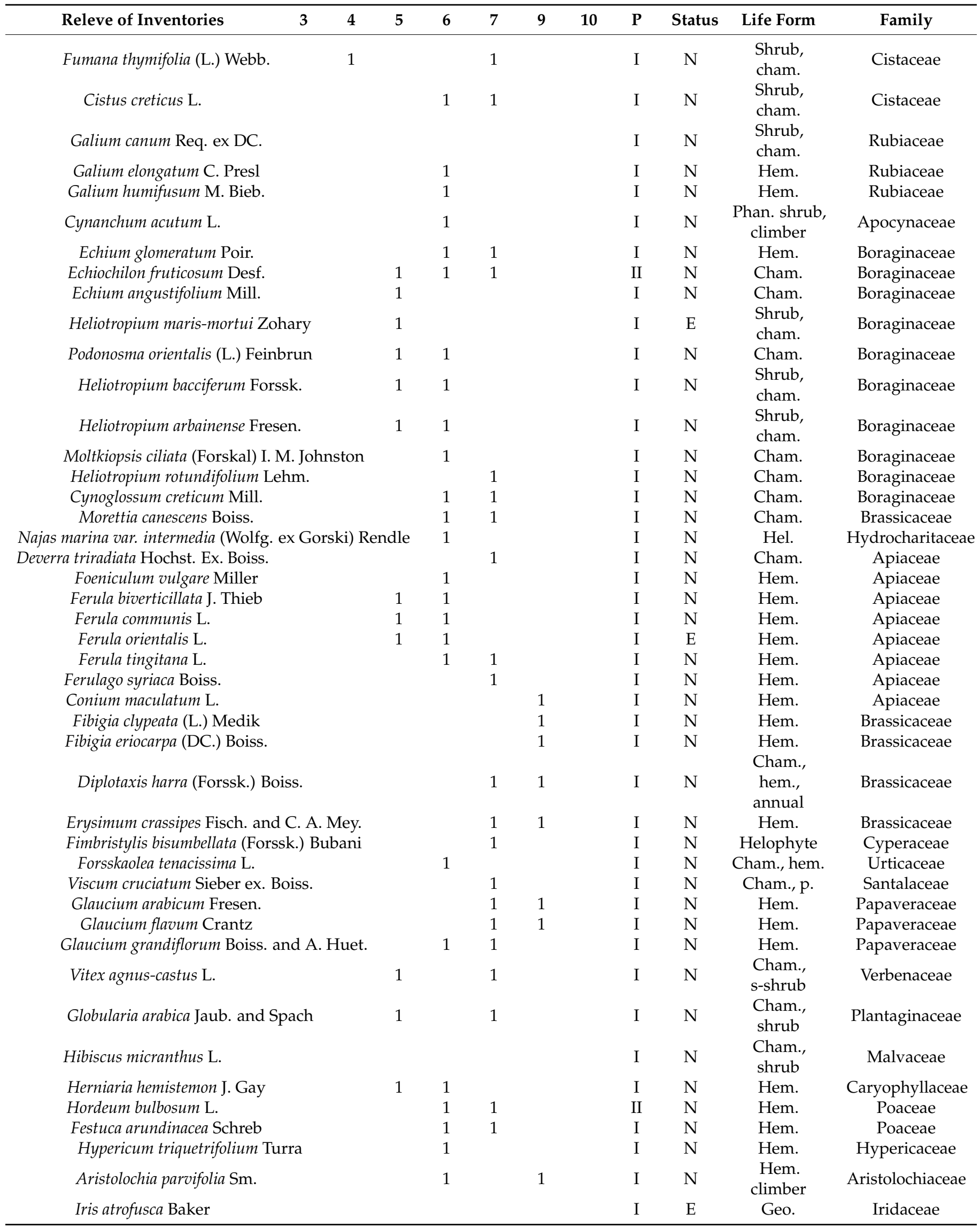


Table 3. Cont.

\begin{tabular}{|c|c|c|c|c|c|c|c|c|c|c|}
\hline Releve of Inventories & 4 & 5 & 6 & 7 & 9 & 10 & $\mathbf{P}$ & Status & Life Form & Family \\
\hline Iris atropurpurea Baker & & & 1 & & & & I & $\mathrm{E}$ & Geo. & Iridaceae \\
\hline Iris palaestina (Bak.) Boiss. & 1 & 1 & 1 & 1 & & & III & $\mathrm{E}$ & Geo. & Iridaceae \\
\hline Iris vartanii Foster & & 1 & & & & & I & $\mathrm{E}$ & Geo. & Iridaceae \\
\hline Gladiolus italicus Mill. & & 1 & & & & & I & $\mathrm{N}$ & Geo. & Iridaceae \\
\hline Juncus acutus L. & & 1 & & & & & I & $\mathrm{N}$ & Hem. & Juncaceae \\
\hline Juncus articulates L. & & & 1 & & & & $\mathrm{I}$ & $\mathrm{N}$ & Hem. & Juncaceae \\
\hline Juncus subulatus Forssk. & & & & 1 & & & I & $\mathrm{N}$ & Hem. & Juncaceae \\
\hline \multirow{2}{*}{\multicolumn{4}{|c|}{$\begin{array}{l}\text { Jacquemontia unilateralis (Roem. and Schult.) O'Donell } \\
\text { Kickxia aegyptiaca (L.) Nabelek }\end{array}$}} & & 1 & & $\mathrm{I}$ & $\mathrm{N}$ & Cham. & Convolvulaceae \\
\hline & & & & & 1 & & $\mathrm{I}$ & $\mathrm{N}$ & Cham. & Plantaginaceae \\
\hline \multicolumn{4}{|l|}{ Kickxia judaica Danin } & & 1 & & I & $\mathrm{E}$ & Cham. & Plantaginaceae \\
\hline \multicolumn{4}{|l|}{ Launaea nudicaulis (L.) Hooker fil. } & 1 & & & $\mathrm{I}$ & $\mathrm{N}$ & Hem. & Compositae \\
\hline \multicolumn{4}{|l|}{ Leontodon tuberosus L. } & 1 & 1 & & I & $\mathrm{N}$ & Hem. & Compositae \\
\hline \multicolumn{4}{|c|}{ Leopoldia bicolor (Boiss.) Eig. and Feinbrun } & 1 & 1 & & $\mathrm{I}$ & $\mathrm{N}$ & Geo. & Asparagaceae \\
\hline \multicolumn{4}{|c|}{ Leptadenia pyrotechnica (Forssk.) Decne. } & 1 & & & $\mathrm{I}$ & $\mathrm{N}$ & Phan. & Apocynaceae \\
\hline \multicolumn{4}{|l|}{ Fimbristylis ferruginea (L.) } & 1 & 1 & & $\mathrm{I}$ & $\mathrm{N}$ & Hel. & Cyperaceae \\
\hline \multicolumn{4}{|l|}{ Verbascum tiberiadis Boiss. } & 1 & 1 & & $\mathrm{I}$ & $\mathrm{N}$ & Hem. & Scrophulariaceae \\
\hline \multicolumn{2}{|l|}{ Verbascum sinaiticum Benth. } & 1 & 1 & 1 & & & II & $\mathrm{N}$ & Hem. & Scrophulariaceae \\
\hline \multicolumn{2}{|l|}{ Verbascum galilaeum Boiss. } & 1 & 1 & 1 & & & II & $\mathrm{N}$ & Hem. & Scrophulariaceae \\
\hline \multicolumn{2}{|l|}{ Verbascumjordanicum Murb. } & 1 & 1 & 1 & & & II & $\mathrm{N}$ & Hem. & Scrophulariaceae \\
\hline \multicolumn{2}{|l|}{ Verbascum gaillardotii Boiss. } & 1 & 1 & 1 & & & II & $\mathrm{N}$ & Hem. & Scrophulariaceae \\
\hline Verbena officinalis L. & 1 & & 1 & 1 & 1 & & III & $\mathrm{N}$ & Hem. & Verbenaceae \\
\hline \multicolumn{2}{|l|}{ Vigna luteola (Jacq.) Benth. } & & & 1 & 1 & & I & $\mathrm{N}$ & $\begin{array}{l}\text { Cham., s-sh., } \\
\text { climber }\end{array}$ & $\begin{array}{l}\text { Leguminosae/ } \\
\text { Papilionaceae }\end{array}$ \\
\hline \multicolumn{2}{|l|}{ Clematis flammula L. } & & & & 1 & & I & $\mathrm{N}$ & Phan., v. & $\begin{array}{l}\text { Anunculaceae/ } \\
\text { Ranunculaceae }\end{array}$ \\
\hline Clematis cirrhosa L. & & & & & 1 & & I & $\mathrm{N}$ & Phan., v. & $\begin{array}{l}\text { Anunculaceae/ } \\
\text { Ranunculaceae }\end{array}$ \\
\hline
\end{tabular}

Abbreviations: T, tree; Phan shrub, phanerophyte shrub; Phan shrub climber, phanerophyte shrub climber; Phan. shrub vine, phanerophyte shrub vine; Phan. vine, phanerophyte vine; Phan. dwarf shrubs, phanerophyte dwarf shrubs; Cham., chamaephyte; Cham. semi-shrubs, chamaephyte semi-shrubs; Sh, cham., chamaephyte shrublet; Cham., Shrub, chamaephyte shrubs; Cham. shrubs climber, chamaephyte shrubs climber; Cham., hem, chamaephyte hemicryptophyte; Cham., p., chamaephyte parasite; Cham., hem., annual, chamaephytehemicryptophyte-annual; Hem., hemicryptophyte; Hem. climber, hemicryptophyte climber; Geo., geophyte; Geo. vine, geophyte vine; and Hel.: helophyte. Percentage of plant species present in the sample studies and communities: $\mathrm{V}=100 \%, \mathrm{IV}=60.1-80 \%$, III $=40.1-60 \%, \mathrm{II}=20.1-40 \%$ and $\mathrm{I}=0.1-20 \%$. N: native, E: endemic Sh: shrub, ASL: association and ASL2: association 2.

The main forests are found in the various mountains and highlands of Palestine, stretching from the heights of the Hebron Mountains in the south to Ras Al-Naqoura, Galilee and Safed in the north. In most of these areas cultivated plants have replaced natural plants for several centuries.

These results show that the study area lies within the region between the Mediterranean Sea and West Asia, and botanists divide Palestinian flora into eight distinct groups, which are: Mediterranean, Eurasia, Euro-Siberian, Irano-Turanian, Sudano-Zambesian, Saharo-Arabian, Americas, Australia and South Africa, as well as plants that grow in Palestine $[16,63,64]$.

Furthermore, the great difference between the pine forests of Pinus halepensis Miller of the western Mediterranean with those existing in Palestine allows us to propose the new alliance Cupresso sempervirentis-Pinus halepensis, with an eastern Mediterranean distribution and dry thermomediterranean environments. As a typus of the Cupresso sempervirentisPinus halepensis alliance we chose the association Cupresso sempervirentis-Pinetum halepensis ass. nova. The alliance was characterized by P. Pinea L., P. brutia Tenore, C. sempervirens L. C. arizonica Greene, T. occidentalis L., J. phoenicea L., J. excelsa M. Bieb. and J. drupacea Labill. [66-68].

The syntaxonomical interpretation of these associations is shown below: 
1. Forest vegetation:

Class: Quercetea ilicis Br.-Bl. ex. A. and O. Bolòs 1950 [69].

Order: Pinetalia halepensis Biondi, Blasi, Galdenzi, Pesaresi et Vagge in Biondi et al. [27] (2014).

Alliance: Cupresso sempervirentis-Pinus halepensis all. nova.

Typus of alliance: Ass. Cupresso sempervirentis-Pinetum halepensis ass. nova.

2. Maquis, macchie and steppe vegetation:

Class: Quercetea calliprini or palaestini nova.

Order: Quercetalia calliprini Zohary [64] 1960.

Alliance: Querco-Pistacion lentisci all. nova.

Association: Pistacio lentisci-Quercetum calliprini ass. nova.

Moreover, forests of pines are found on different geological formations in the world, including in the Mediterranean, Europe and different regions in the Palestine mountains. P. halepensis Miller, P. Pinea L., P. canariensis C. Smith, P. brutia Tenore and Cupressus genus as C. sempervirens L., C. arizonica Greene, T. occidentalis L., J. phoenicea L., J. excelsa M. Bieb. and J. drupacea Labill, C. equesitifolia L., C. sempervirentis and P. halepensis associations have been described by many researchers in antecedent studies [16-19]. Phytogeographically, plant associations belonging to the forest flora that extend over Europe, the Mediterranean and from the north to the south of Palestine were included within the classes of Quercetea ilicis [69]. Pine and Cupressus forests are placed under two different alliances: the order Pinetalia halepensis, Biondi et al. 2014 [27], and the alliance Juniperon phoeniceae-Pinus acutisquamae and Quercetea ilicis [65]. The components of the alliance of the Pinus halepensis [64] order of Pinetalia halepensis [27] are apparent in this association due to the range of anthropogenic harm to the forest steppes and mountain zones as a result of some military activities for the purpose of training and fire, in addition to the existence of numerous plants that return to this association.

\subsubsection{Pistacio lentisci-Quercetum calliprini ass. nova}

The second association includes represented inventories $(3,4,6,7,9,10$ and 5) of the principal component analysis in (Table 3, typus inv. 1); the community grows in areas of the Beit Jibrin in the dry, infra- and thermotropical thermotypes. This association is a composition of Q. calliprinos Webb. (Oak Palestine, Q. palaetina Kotschy), Q. inthaburensis Decne., Q. infectoria Olivier, Q. boissieri Reut., Q. cerris L., A. obtusifolium smith, A. monspessulanum L., Q. libani G. Olivier, Q. look Kotschy, Q. boissieri Reut., and P. lentiscus L. P. palaestina Boiss., accompanied with R. palaestinus Boiss., R. disperma Ehrenb. ex Boiss., R. alaternus L., C. siliqua L. and P. khinjuk Stocks species, and it belong to Mediterranean macchie vegetation, evergreen Mediterranean forests and deciduous Mediterranean forests. The soil of this association has a partially basic character, low organic matter and a medium of clayey-loamy texture. Due to the high degradation, numerous steppes and xerophilous species permeated into the floristic structure of this association. Quercus as Oak extends from the eumediterranean and submediterranean regions (Quercetalia ilicis), according to a Braun-Blanquet rating [69-73], and many Quercus species, such as $Q$. calliprinos Webb., Q. inthaburensis Decne, Q. infectoria Olivier, Q. libani G. Olivier, Q. look Kotschy and Q. boissieri Reut. associations were qualified in neighboring regions and studied. Likewise, the Quercus genus association described in Palestine in the highland mountains and west of Hebron [16-19] was categorized under the Quercetalia calliprini order. The floristic structure of this association is well-specified by the characteristic species of the order Quercetalia calliprini [63] and a new class, Quercetea calliprini or palaestini. For these causes, the association must be included in the syntax unity aforementioned. However, the second association is dominated by $Q$. calliprinos Webb. (Q. palaestina Kotschy or Q. coccifera L.), Q. inthaburensis Decne., Q. infectoria Olivier, Q. boissieri Reut., Q. cerris L., Q. look Kotschy, Q. libani G. Olivier, C. siliqua L., S. junceum L., C. siliquastrum L., P. gillesii Hook, A. salicina Lindl., A. cyanophylla Lindl., C. equesitifolia L., S. japonica L., C. villosa (Poir.) Link, R. raetam (Forssk.) Webb. and Berthel., G. monspessulana (L.) O. Bolós and Vigo., A. radiana Savi., P. palaestina Boiss., P. khinjuk Stocks, P. lentiscus 
L., P. saportae Burnat., P. atlantica Desf., P. terebinthus L., P. vera L., S. molle L., R. coriaria L., R. palaestinus Boiss., (R. lycioides L.), R. alaternus L., Z. Spina-christi L. Desf., Z. Lotus (L.) Lam., P. spina-christi Miller, Z. jujuba Miller, S. tripartita (Ucria) Moffett, S. thea (Osbeck) M.C. Johnst., M. germanica L., C. azarolus L., A. communis L, C. oriana (L.) DC., S. spinosum (L.) Spach, P. syriaca Boiss., C. monogyna Jacq., M. communis Desf., P. coccinea M. Roem., P. spinosa L., F. retusa L., F. sycomorus L., F. cariaca L., M. alba L., M. nigara L., O. europaea L., P. media L., O. oleaster Hoffmanns. and Link, A. obtusifolium Sm. or A. syriscum Boiss., A. monspessulanum L., F. retusa L., F. sycomorus L., F. cariaca L., M. alba L., M. nigara L., O. ficus-indica (L.) Miller, O. robusta J.C. Wendl., O. ficus-barbarica A. Berger, S. alba L., P. alba L., P. nigra L., P. euphratica Oliver, etc.

Forest oaks and maquis evergreen vegetation, such as Q. calliprinos Webb. (Q. palaestina K.), Q. inthaburensis Decne, Q. infectoria Olivier, Q. boissieri Reut., Q. cerris L., P. palaestina Boiss., R. coriaria L., C. siliqua L., S. junceum L., P. gillesii Hook, A. salicina Lindl., A. cyanophylla Lindl., S. japonica L., R. raetam (Forssk.) Webb. and Berthel., G. monspessulana (L.) O. Bolós and Vigo, A. radiana Savi. and O. europaea L., a forest growing in a granular climate community in a habitat, include Mediterranean scrubs, steppes, grasslands, desert oases, urban areas, forest and canyons environments with dry sub-humid regions and an infrared thermal Mediterranean pattern to the mesomediterranean, on limestone composed of grain skeletal fragment organisms and organic matter. Therefore, the floristic arrangement of this association (Ceratonia and Quercus species) in the Mediterranean and Middle East regions extends from the eumediterranean to the Eurasian regions (Quercetalia ilicis), corresponding to its Braun-Blanquet rating [69-73]. Several Quercus and Ceratonia species, such as Q. look Kotschy, Q. boissieri Reut., Q. calliprinos (Q. coccifera L. or Q. palaestina K.), C. siliqua L. [74]., S. junceum L., P. gillesii Hook, A. salicina Lindl., A. cyanophylla Lindl., R. raetam (Forssk.) Webb. and Berthel. and $A$. radiana Savi. associations were discovered in neighboring regions and studied [16,17]. In the same way, the Quercus and Ceratonia species, such as the Q. look Kotschy and C. siliqua L. associations described in Southern Palestine, as well as to the west of the Hebron area by Ighbareyeh et al. [16], were proposed a new classification under the Quercetalia calliprini order and Querco-istacion lentisci alliance. Consequently, the floristic makeup of this association is well-identified by the specific species of the Quercetalia calliprini order and the Quercetea calliprini or palaestini class; for these causes, the association must be included in the syntaxa unity mentioned. Furthermore, we suggested a new alliance (Querco-Pistacion lentisci), order (Quercetalia calliprini) [64] and class (Quercetea calliprini or Quercetea palaestini), in addition to the Quercetalia ilicis order. The following are diagnostic class species (subordinated units) and vascular plants: Q. calliprinos Webb. (Q. palaestina K.), Q. inthaburensis Decne., Q. infectoria Olivier, Q. boissieri Reut., Q. cerris L., Q. look Kotschy, Q. libani G. Olivier, C. siliqua L., S. junceum L., C. siliquastrum L., P. gillesii Hook, A. salicina Lindl. A. cyanophylla Lindl., S. japonica L., C. villosa (Poir.) Link, $R$. raetam (Forssk.) Webb. and Berthel., G. monspessulana (L.) O. Bolós and Vigo, A. radiana Savi., P. palaestina Boiss., P. khinjuk Stocks, P. lentiscus L., P. saportae Burnat., P. atlantica Desf., P. terebinthus L., P. vera L., S. molle L., R. coriaria L., R. palaestinus Boiss. (R. lycioides L.), R. alaternus L., Z. Spina-christi L. Desf., Z. Lotus (L.) Lam., P. spina-christi Miller, Z. jujuba Miller, S. tripartita (Ucria) Moffett, S. thea (Osbeck) M.C.Johnst., M. germanica L., C. azarolus L., A. communis L., C. oriana (L.) DC., S. spinosum (L.) Spach, P. syriaca Boiss., C. monogyna Jacq., M. communis Desf., P. coccinea M. Roem., P. spinosa L., F. retusa L., F. sycomorus L., F. cariaca L., F. benjamina L.. M. alba L., M. nigara L., O. europaea L., P. media L., O. oleaster Hoffmanns. and Link, A. obtusifolium Sm. or A. syriscum Boiss., A. monspessulanum L., P. halepensis Miller, P. P. L., P. canariensis C. Smith, P. brutia Tenor, C. sempervirens L., C. sempervirens L. var. horizontalis Miller, C. arizonica Greene, T. occidentalis L., J. phoenicea L., J. excelsa M. Bieb., J. drupacea Labill., A. monspessulanum L., F. retusa L., F. sycomorus L., F. cariaca L., M. alba L., M. nigara L., O. ficus indica (L.) Miller, O. robusta J.C. Wendl., O. ficus-barbarica A. Berger, S. alba L., P. alba L., P. nigra L., P. euphratica Oliver, etc.

On the other hand, for the flora and vegetation, we found more than 72 families and 800 species of plants including forest oak, maquis, woodland, scrub evergreen, macchie and 
steppe land Quercus species, such as Q. calliprinos Webb. (Q. palaestina k., Q. inthaburensis Decne., Q. infectoria Olivier, Q. cerris L., etc., and the Pistachio genus, such as species of $P$. lentiscus L., R. palaestinus Boiss. (R. lycioides L.), R. alaternus L., Z. Spina-christi L. and many macchie and steppes, shrubs, herbaceous and landscape vegetation, such as $A$. spinosa L., R. palaestinum Feinbrun, A.s foetida L., C. abyssinica Kunth and Bouche, L. barbarum L., $L$. europaeum L., N. glauca Graham, L. depressum Stocks, L. schweinfurthii Dammer, L. shawii Roem. and Schult., S. sinaicum Boiss., S. incanum L., P. pungens Willd., P. brachyodon (Boiss.) Zohary, P. chrysophylla Boiss., B. saxatilis Sieber ex. C. Presl., B. philistaea Bornm., B. undulata (Sieber ex Fresen.) Bentham, B. arabica (Boiss.) Maire and Weiller, P. platystegia Post., P. viscosa Poiret., S. eigii Zohary, S. dominica L., S. lanigera Poir., S. thymbra L., S. thymbrifolia Hedge and Feinbrun, S. palaestina L., S. fruticosa Miller, S. officinalis L., S. aegyptiaca L., S. palaestina Benth., S. aethiopis L., M. fruticosa (L.) Druce., T. capitatum L., T. creticum L., T. capitata (L.) Cav., T. spicata L., B. populneus (Schott and Endl.) R.Br., J. mimosaefolia D. Don., C. spinosa L., C. sicula Duh., C. aegyptia Lam., C. australis L., A. halimus L., N. mucronata (Forssk.) Asch. and Schweinf., H. persicum Bunge, H. negevensis (Iljin and Zohary) L. Boulos, S. fruticosa (L.) L., A. javanica (Burm.f.) Juss. ex Schult., S. imbricata Forssk., S. cyclophylla Baker, A. macrostachyum (Moric.) Piirainen and G. Kadereit, S. palaestina Eig. and Zohary, H. lancifolius (Boiss.) Kothe-Heinr., H. salicornicum (Moq.) Bunge ex Boiss., O. natrix L., A. andrachne L., L. nobilis L., P. aquilinum (L.) Kuhn, M. azedarach L., P. mascula (L.) Miller, A. filiculoides Lam., L. pyrotechnica (Forssk.) Decne., S. officinalis L., A. aleppica DC., G. tournefortii L., A. arborescens L., A. monosperma Delile, A. garcinii (Burm.f.) DC., $P$. dioscoridis (L.) DC., A. sieberi Besser., A. horridus L., A. palaestinus Baker, S. asperan L., P. aculeata L., O. baccatus Delile, N. oleander L., C. acutum L., P. aphylla Decne., M. peregrina (Forssk.) Fiori, G. villosa Willd, E. aphylla Forskal, E. foeminea Forssk., A. halimus L., A. setifera Moq., A. syriaca Iljin, A. orientalis (L.) Boiss., A. strigosa Boiss. and Hohen., A. galilaea Boiss., A. tinctoria (L.) Tausch, A. altissima (Miller) Swingle, $R$. tinctorum L., R. tenuifolia D'Urv., R. chalepensis L., T. hirsuta (L.) Endl., V. eremobium Murb., V. fruticulosum Post., H. helix L., E. crassifolium L'Her., E. glaucophyllum (L.) L'Hér., E. arborescens (Desf.) Willd., E. acaule (L.) Becherer and Thell., E. creticum Lam., E. falcatum F. Delaroche, E. glomeratum Lam., E. maritimum L., E. cannabinum L., E. hierosolymitana Boiss., E. hirta L., E. hirsuta L., E. terracina L., B. aegyptiaca (L.) Delile, Z. dumosum Boiss., N. retusa (Forssk.) Ascherson, F. bruguieri DC., F. mollis Delile, F. orientalis C. Presl., Fagonia arabica L., C. arabica (Boiss.) Diagn. P1. Orient, C. lanatus Vahl., C colocynthis (L.) Schrader, C. dorycnium L., I. cairica (L.) Sweet, I. imperati (Vahl.) Griseb., H. aureus L., P. orientalis (L.) Feinbrun, M. myrtifolia Boiss. et Hohen., M. nervosa (Desf.) Benth., C. insulare (Candargy) Govaerts, E. cannabinum L., Doellia bovei (DC.) Anderb., H. sanguineum (L.) Kostel., I. maris-mortui Feinbrun, C. iphionoides (Boiss. and Blanche) Brul., C. tinctoria (L.) J. Gay, C. hierosolymitana Boiss., C. reuteriana Boiss., C. syriaca Boiss., E. philistaeus Feinbrun and Zohary, V. vilosa Roth., F. thymifolia (L.) Webb., C. creticus L., G. canum Req. ex. DC., G. elongatum C. Presl., G. humifusum M. Bieb., C. acutum L., E. glomeratum Poir., E. fruticosum Desf., E. angustifolium Mill., H. maris-mortui Zohary, P. orientalis (L.) Feinbrun, H. bacciferum Forssk., H. arbainense Fresen., M. ciliata (Forskal) I. M. Johnston, H. rotundifolium Lehm., C. creticum Mill., M. canescens Boiss., $N$. marina var. intermedia (Wolfg. ex Gorski) Rendle, D. triradiata Hochst. Ex. Boiss., F. vulgare Miller, F. biverticillata J. Thieb, F. communis L., F. orientalis L., F. tingitana L., F. syriaca Boiss., C. maculatum L., F. clypeata (L.) Medik, F. eriocarpa (DC.) Boiss., D. harra (Forssk.) Boiss., E. crassipes Fisch. and C. A. Mey., F. bisumbellata (Forssk.) Bubani, F. tenacissima L., V. cruciatum Sieber ex. Boiss., G. arabicum Fresen., G. flavum Crantz, G. grandiflorum Boiss. and A. Huet., V. agnus-castus L., G. arabica Jaub. and Spach, H. micranthus L., H. hemistemon J. Gay, $H$. bulbosum L., F. arundinacea Schreb., H. triquetrifolium Turra, A. parvifolia Sm., I. atrofusca Baker, I. atropurpurea Baker, I. palaestina (Baker) Boiss., I. vartanii Foster, G. italicus Miller, J. acutus L., J. articulates L., J. subulatus Forssk., J. unilateralis (Roem. and Schult.) O'Donell, K. aegyptiaca (L.) Nabelek, K. judaica Danin, L. nudicaulis (L.) Hooker fil., L. tuberosus L., L. bicolor (Boiss.) Eig. and Feinbrun, L. pyrotechnica (Forssk.) Decne., F. ferruginea (L.), $V$. tiberiadis Boiss., V. sinaiticum Benth., V. galilaeum Boiss., V. jordanicum Murb., V. gaillardotii 
Boiss., V. officinalis L., V. luteola (Jacq.) Benth., C. monogyna Vahl., C. epithymum (L.), C. pedicellata Ledeb., C. planiflora Ten., C. palaestina Boiss. and many other species. For the Quercetalia calliprinii order [63], we found characteristic species such as Quercus spp., $Q$. calliprinos Webb. or Q. palaestina K., Q. inthaburensis Decne., Q. boissieri Reut., Q. infectoria Olivier, Q. cerris L., Q. look Kotschy, Q. libani G. Olivier, C. siliqua L., S. junceum L., F. cariaca L., M. alba L., M. nigara L., O. ficus indica (L.) Miller, C. siliquastrum L., P. gillesii Hook, A. cyanophylla Lindl., S. japonica L., C. villosa (Poir.) Link, etc. Species characteristic of the alliance (Querco-Pistacion lentisci) were also found, such as P. lentiscus L., P. palaestina Boiss., P. khinjuk Stocks, P. saportae Burnat., P. vera L., P. atlantica Desf., P. terebinthus L., S. molle L., R. coriaria L., R. palaestinus Boiss. (R. lycioides L.), R. alaternus L., Z. Lotus (L.) Lam., Z. Spina-christi L. Desf., P. spina-christi Miller, Z. jujuba Miller, S. tripartita (Ucria.) Moffett., $S$. thea (Osbeck) M.C.Johnst., M. germanica L., C. azarolus L., A. communis L, C. oriana (L.) DC., S. spinosum (L.) Spach, P. syriaca Boiss., C. monogyna Jacq., M. communis Desf., P. coccinea M. Roem., P. spinosa L., F. retusa L., F. sycomorus L., F. cariaca L., M. alba L., M. nigara L., O. europaea L., P. media L., O. oleaster Hoffmanns. and Link, A. obtusifolium Sm. or A. syriscum Boiss., A. monspessulanum L., etc.

However, the ecological characteristics, chorotypes, habitats, climatology and plant geography distributions in this proposed class have been studied, as shown in the fourth table (Table 4), where the plants are distributed into deserts, shrub-steppes, semi-steppe shrublands and Mediterranean woodlands and shrublands, in addition to Mediterranean maquis and forests.

Table 4. Ecological and habitat characteristics of distributed plants.

\begin{tabular}{|c|c|c|c|c|}
\hline Species & Chorotype & Habitat & Distribution & Climate \\
\hline $\begin{array}{l}\text { Quercus calliprinos Webb. } \\
\text { (Quercus palaestina K., } \\
\text { Quercus coccifera L.) }\end{array}$ & M & $\begin{array}{l}\text { Mediterranean maquis } \\
\text { and forests }\end{array}$ & $\begin{array}{l}\text { Mediterranean woodlands and } \\
\text { shrublands, semi-steppe } \\
\text { shrublands, Mt. Hermon }\end{array}$ & M \\
\hline $\begin{array}{l}\text { Quercus inthaburensis } \\
\text { Decne. }\end{array}$ & M & $\begin{array}{l}\text { Mediterranean maquis } \\
\text { and forests }\end{array}$ & $\begin{array}{l}\text { Mediterranean woodlands } \\
\text { and shrublands }\end{array}$ & M \\
\hline Quercus infectoria Olivier & M, IT & $\begin{array}{l}\text { Mediterranean maquis } \\
\text { and forests }\end{array}$ & $\begin{array}{l}\text { Mediterranean woodlands } \\
\text { and shrublands }\end{array}$ & M \\
\hline Quercus look Kotschy & Oro-Mediterranean & $\begin{array}{l}\text { Tragacanth shrub } \\
\text { vegetation }\end{array}$ & Oro-Mediterranean & M \\
\hline Quercus libani G. Olivier & Oro-Mediterranean & $\begin{array}{l}\text { Tragacanth shrub } \\
\text { vegetation }\end{array}$ & Oro-Mediterranean & M \\
\hline $\begin{array}{l}\text { Quercus boissieri Reut. } \\
\text { (Quercus boissieri Reut. var. } \\
\text { latifolia (Boiss.) Zohary) }\end{array}$ & M, IT & $\begin{array}{l}\text { Mediterranean maquis } \\
\text { and forests }\end{array}$ & $\begin{array}{l}\text { Mediterranean woodlands } \\
\text { and shrublands }\end{array}$ & M \\
\hline Quercus cerris L. & M & $\begin{array}{l}\text { Mediterranean maquis } \\
\text { and forests }\end{array}$ & $\begin{array}{l}\text { Mediterranean woodlands } \\
\text { and shrublands }\end{array}$ & M \\
\hline Pistacia palaestina Boiss. & M & $\begin{array}{l}\text { Mediterranean maquis } \\
\text { and forests }\end{array}$ & $\begin{array}{l}\text { Mediterranean woodlands and } \\
\text { shrublands, semi-steppe } \\
\text { shrublands }\end{array}$ & M \\
\hline Pistacia saportae Burnat. & M & $\begin{array}{l}\text { Mediterranean maquis } \\
\text { and forests }\end{array}$ & $\begin{array}{l}\text { Mediterranean woodlands and } \\
\text { shrublands, semi-steppe } \\
\text { shrublands }\end{array}$ & M \\
\hline Pistacia lentiscus L. & M & $\begin{array}{l}\text { Mediterranean maquis } \\
\text { and forests }\end{array}$ & $\begin{array}{l}\text { Mediterranean woodlands and } \\
\text { shrublands, semi-steppe } \\
\text { shrublands }\end{array}$ & M \\
\hline Rhamnus palaestinus Boiss. & M & $\begin{array}{l}\text { Mediterranean maquis } \\
\text { and forests }\end{array}$ & $\begin{array}{l}\text { Mediterranean woodlands and } \\
\text { shrublands, semi-steppe } \\
\text { shrublands }\end{array}$ & M, Tr, SD \\
\hline $\begin{array}{l}\text { Rhamnus disperma } \\
\text { Ehrenb.ex. Boiss. }\end{array}$ & M & $\begin{array}{l}\text { Mediterranean maquis } \\
\text { and forests }\end{array}$ & $\begin{array}{l}\text { Mediterranean woodlands and } \\
\text { shrublands, semi-steppe } \\
\text { shrublands }\end{array}$ & SD, ED \\
\hline Rhamnus alaternus L. & M & $\begin{array}{l}\text { Mediterranean maquis } \\
\text { and forests }\end{array}$ & $\begin{array}{l}\text { Mediterranean woodlands } \\
\text { and shrublands }\end{array}$ & M \\
\hline
\end{tabular}


Table 4. Cont.

\begin{tabular}{|c|c|c|c|c|}
\hline Species & Chorotype & Habitat & Distribution & Climate \\
\hline Ceratonia siliqua $\mathrm{L}$ & M & $\begin{array}{l}\text { Mediterranean maquis } \\
\text { and forests }\end{array}$ & $\begin{array}{l}\text { Mediterranean woodlands and } \\
\text { shrublands, semi-steppe } \\
\text { shrublands }\end{array}$ & M \\
\hline Pistacia atlantica Desf. & IT & $\begin{array}{l}\text { Mediterranean maquis } \\
\text { and forest, hard } \\
\text { rock outcrops }\end{array}$ & $\begin{array}{l}\text { Mediterranean woodlands and } \\
\text { shrublands, semi-steppe } \\
\text { shrublands }\end{array}$ & $\mathrm{M}, \mathrm{Tr}, \mathrm{SD}$ \\
\hline Pistacia khinjuk Stocks & IT & $\begin{array}{l}\text { Mediterranean maquis } \\
\text { and forests, hard } \\
\text { rock outcrops }\end{array}$ & $\begin{array}{l}\text { Mediterranean woodlands and } \\
\text { shrublands, semi-steppe } \\
\text { shrublands }\end{array}$ & M \\
\hline Schinus rip L. & A & $\begin{array}{l}\text { Mediterranean maquis } \\
\text { and forests, hard } \\
\text { rock outcrops }\end{array}$ & $\begin{array}{l}\text { Mediterranean woodlands } \\
\text { and shrublands }\end{array}$ & M \\
\hline Rhus coriaria L. & IT & $\begin{array}{l}\text { Mediterranean maquis } \\
\text { and forests, hard } \\
\text { rock outcrops }\end{array}$ & $\begin{array}{l}\text { Mediterranean woodlands } \\
\text { and shrublands, } \\
\text { semi-steppe shrublands }\end{array}$ & M \\
\hline $\begin{array}{l}\text { Schinus terebinthifolius } \\
\text { Raddi. }\end{array}$ & A & $\begin{array}{l}\text { Mediterranean maquis } \\
\text { and forests, hard } \\
\text { rock outcrops }\end{array}$ & $\begin{array}{l}\text { Mediterranean woodlands } \\
\text { and shrublands, desert, } \\
\text { semi-steppe shrublands }\end{array}$ & $\mathrm{M}, \mathrm{Tr}, \mathrm{SD}$ \\
\hline $\begin{array}{l}\text { Rhus ripartite } \\
\text { (Ucria) Grande }\end{array}$ & M, SA & Hard rock outcrops & $\begin{array}{l}\text { Deserts, shrub-steppes, } \\
\text { semi-steppe shrublands, } \\
\text { Mediterranean woodlands } \\
\text { and shrublands }\end{array}$ & $\mathrm{Tr}, \mathrm{SD}$ \\
\hline $\begin{array}{l}\text { Zizyphus Spina-christi } \\
\text { L. Desf. }\end{array}$ & SA & $\begin{array}{c}\text { Humid habitats, deserts, } \\
\text { shrub-steppes, } \\
\text { Mediterranean } \\
\text { grasslands, } \\
\text { thermophilous plants }\end{array}$ & $\begin{array}{l}\text { Deserts, shrub-steppes, } \\
\text { semi-steppe shrublands, } \\
\text { Mediterranean woodlands } \\
\text { and shrublands }\end{array}$ & $\mathrm{M}, \mathrm{Tr}, \mathrm{SD}, \mathrm{ED}$ \\
\hline Zizyphus Lotus (L.) Lam. & M, SA & Batha, phrygana & $\begin{array}{l}\text { Mediterranean woodlands } \\
\text { and shrublands, } \\
\text { semi-steppe shrublands }\end{array}$ & $\mathrm{Tr}, \mathrm{SD}$ \\
\hline Paliurus spina-christi Miller & M, IT & $\begin{array}{l}\text { Mediterranean maquis } \\
\text { and forests, hard } \\
\text { rock outcrops }\end{array}$ & $\begin{array}{l}\text { Mediterranean woodlands } \\
\text { and shrublands }\end{array}$ & M \\
\hline Ziziphus jujuba Miller & $S$ & $\begin{array}{c}\text { Humid habitats, deserts, } \\
\text { shrub-steppes, } \\
\text { Mediterranean } \\
\text { grasslands, } \\
\text { thermophilous plants }\end{array}$ & $\begin{array}{l}\text { Deserts, shrub-steppes, } \\
\text { semi-steppe shrublands, } \\
\text { Mediterranean woodlands } \\
\text { and shrublands }\end{array}$ & M, Tr, SD, ED \\
\hline $\begin{array}{c}\text { Sageretia thea (Osbeck) } \\
\text { M. C. Johnst. }\end{array}$ & IT & Cleavages of hard rocks & $\begin{array}{l}\text { Deserts, shrub-steppes, } \\
\text { semi-steppe shrublands }\end{array}$ & $\operatorname{Tr} \mathrm{SD}$ \\
\hline Arbutus unedo L. & M & $\begin{array}{l}\text { Mediterranean maquis } \\
\text { and forests, hard } \\
\text { rock outcrops }\end{array}$ & $\begin{array}{l}\text { Mediterranean woodlands } \\
\text { and shrublands }\end{array}$ & M \\
\hline Mespilus germanica L. & M & $\begin{array}{l}\text { Mediterranean maquis } \\
\text { and forests, hard } \\
\text { rock outcrops }\end{array}$ & $\begin{array}{l}\text { Mediterranean woodlands } \\
\text { and shrublands }\end{array}$ & M \\
\hline Crataegus azarolus L. & M & $\begin{array}{l}\text { Mediterranean maquis } \\
\text { and forests, hard } \\
\text { rock outcrops }\end{array}$ & $\begin{array}{l}\text { Mediterranean woodlands } \\
\text { and shrublands }\end{array}$ & M \\
\hline $\begin{array}{c}\text { Amygdalus ramonensis } \\
\text { Danin }\end{array}$ & IT & Hard rock outcrops & Shrub-steppes & $\mathrm{TZ}, \mathrm{SD}$ \\
\hline $\begin{array}{l}\text { Prunus dulcis (Mill.) } \\
\text { D. A. Webb. }\end{array}$ & M, IT & $\begin{array}{l}\text { Mediterranean maquis } \\
\text { and forests, hard } \\
\text { rock outcrops }\end{array}$ & $\begin{array}{l}\text { Mediterranean woodlands } \\
\text { and shrublands }\end{array}$ & M \\
\hline Crataegus oriana (L.) DC. & M & $\begin{array}{l}\text { Mediterranean maquis } \\
\text { and forests, hard } \\
\text { rock outcrops }\end{array}$ & $\begin{array}{l}\text { Mediterranean woodlands } \\
\text { and shrublands }\end{array}$ & M \\
\hline
\end{tabular}


Table 4. Cont.

\begin{tabular}{|c|c|c|c|c|}
\hline Species & Chorotype & Habitat & Distribution & Climate \\
\hline $\begin{array}{l}\text { Sarcopoterium spinosum (L.) } \\
\text { Spach (Poterium } \\
\text { spinosum L.) }\end{array}$ & M & Batha, phrygana & $\begin{array}{l}\text { Shrub-steppes, deserts, Mt. } \\
\text { Hermon, semi-steppe } \\
\text { shrublands, Mediterranean } \\
\text { woodlands and shrublands }\end{array}$ & M \\
\hline Pyrus syriaca Boiss. & M, IT & $\begin{array}{l}\text { Mediterranean maquis } \\
\text { and forests, hard } \\
\text { rock outcrops }\end{array}$ & $\begin{array}{l}\text { Mediterranean woodlands and } \\
\text { shrublands, semi-steppe } \\
\text { shrublands, montane } \\
\text { vegetation of Mt. Hermon }\end{array}$ & M \\
\hline Crataegus monogyna Jacq. & M, ES & $\begin{array}{l}\text { Mediterranean maquis } \\
\text { and forests, hard } \\
\text { rock outcrops }\end{array}$ & $\begin{array}{l}\text { Mediterranean woodlands } \\
\text { and shrublands }\end{array}$ & M \\
\hline Malus communis Desf. & M & $\begin{array}{l}\text { Mediterranean maquis } \\
\text { and forests, hard } \\
\text { rock outcrops }\end{array}$ & $\begin{array}{l}\text { Mediterranean woodlands } \\
\text { and shrublands }\end{array}$ & M \\
\hline $\begin{array}{l}\text { Pyracantha coccinea } \\
\text { M. Roem. }\end{array}$ & M, ES & $\begin{array}{l}\text { Mediterranean maquis } \\
\text { and forests, hard } \\
\text { rock outcrops }\end{array}$ & $\begin{array}{l}\text { Mediterranean woodlands } \\
\text { and shrublands }\end{array}$ & M \\
\hline Prunus ursina Kotschy & M & $\begin{array}{l}\text { Mediterranean maquis } \\
\text { and forest and hard } \\
\text { rock outcrops }\end{array}$ & $\begin{array}{l}\text { Mediterranean woodlands } \\
\text { and shrublands }\end{array}$ & M \\
\hline $\begin{array}{l}\text { Prunus korshinskyi } \\
\text { Hand.-Mazz. }\end{array}$ & M & $\begin{array}{l}\text { Mediterranean maquis } \\
\text { and forest and hard } \\
\text { rock outcrops }\end{array}$ & $\begin{array}{l}\text { Mediterranean woodlands } \\
\text { and shrublands }\end{array}$ & M \\
\hline $\begin{array}{l}\text { Prunus arabica } \\
\text { (Olivier) Meikle. }\end{array}$ & IT & $\begin{array}{l}\text { Mediterranean maquis } \\
\text { and forest and hard } \\
\text { rock outcrops }\end{array}$ & $\begin{array}{l}\text { Mediterranean woodlands } \\
\text { and shrublands }\end{array}$ & $\operatorname{Tr}$ \\
\hline Rubus sanguineus Friv. & M, IT & Humid habitats & $\begin{array}{l}\text { Mediterranean woodlands and } \\
\text { shrublands, semi-steppe } \\
\text { shrublands, montane } \\
\text { vegetation of Mt. Hermon }\end{array}$ & $\mathrm{M}, \mathrm{ED}$ \\
\hline Pinus halepensis (L.) Miller & M & $\begin{array}{l}\text { Mediterranean maquis } \\
\text { and forests, hard } \\
\text { rock outcrops }\end{array}$ & $\begin{array}{l}\text { Mediterranean woodlands and } \\
\text { shrublands, semi-steppe } \\
\text { shrublands, montane } \\
\text { vegetation of Mt. Hermon }\end{array}$ & M \\
\hline Cupressus sempervirens L. & M & Hard rock outcrops & $\begin{array}{l}\text { Mediterranean woodlands } \\
\text { and shrublands }\end{array}$ & M \\
\hline Pinus pinea $\mathrm{L}$. & EP-EC & Disturbed habitats & $\begin{array}{l}\text { Mediterranean woodlands } \\
\text { and shrublands }\end{array}$ & M \\
\hline Pinus canariensis C. Smith & M & $\begin{array}{l}\text { Mediterranean maquis } \\
\text { and forests, hard } \\
\text { rock outcrops }\end{array}$ & $\begin{array}{l}\text { Mediterranean woodlands } \\
\text { and shrublands }\end{array}$ & M \\
\hline Pinus brutia Tenore & EP-EC & $\begin{array}{l}\text { Mediterranean maquis } \\
\text { and forests, hard } \\
\text { rock outcrops }\end{array}$ & $\begin{array}{l}\text { Mediterranean woodlands } \\
\text { and shrublands }\end{array}$ & M \\
\hline Cupressus arizonica Greene & M & $\begin{array}{l}\text { Mediterranean maquis } \\
\text { and forests, hard } \\
\text { rock outcrops }\end{array}$ & $\begin{array}{l}\text { Mediterranean woodlands } \\
\text { and shrublands }\end{array}$ & M \\
\hline $\begin{array}{l}\text { Seidliziz rosmarinus } \\
\text { Buge ex. Boiss. }\end{array}$ & SA & Deserts, salty habitats & $\begin{array}{l}\text { Deserts, shrub-steppes, } \\
\text { semi-steppe shrublands }\end{array}$ & SA, SD \\
\hline Juniperus phoenicea L. & M & $\begin{array}{l}\text { Hard rock outcrops, } \\
\text { shrub-steppes }\end{array}$ & $\begin{array}{l}\text { Mediterranean woodlands } \\
\text { and shrublands }\end{array}$ & M \\
\hline Juniperus excelsa M. Bieb. & M, ES & $\begin{array}{l}\text { Tragacanth shrub } \\
\text { vegetation } \\
\text { (Oro-Mediterranean) }\end{array}$ & $\begin{array}{l}\text { Tragacanth shrub vegetation } \\
\text { (Oro-Mediterranean) }\end{array}$ & M \\
\hline Juniperus drupacea Labill. & M & $\begin{array}{l}\text { Tragacanth shrub } \\
\text { vegetation } \\
\text { (Oro-Mediterranean) }\end{array}$ & $\begin{array}{l}\text { Mt. Hermon, Mediterranean } \\
\text { woodlands and shrublands }\end{array}$ & M \\
\hline
\end{tabular}


Table 4. Cont.

\begin{tabular}{|c|c|c|c|c|}
\hline Species & Chorotype & Habitat & Distribution & Climate \\
\hline $\begin{array}{c}\text { Spartium junceum L. } \\
\text { (Genista juncea (L.) Scop.) }\end{array}$ & M & $\begin{array}{l}\text { Mediterranean maquis } \\
\text { and forests, hard } \\
\text { rock outcrops }\end{array}$ & $\begin{array}{c}\text { Mediterranean woodlands and } \\
\text { shrublands, montane } \\
\text { vegetation of Mt. Hermon }\end{array}$ & M \\
\hline Cersis siliquastrum L. & M & $\begin{array}{l}\text { Mediterranean maquis } \\
\text { and forests, hard } \\
\text { rock outcrops }\end{array}$ & $\begin{array}{l}\text { Mt. Hermon, Mediterranean } \\
\text { woodlands and shrublands }\end{array}$ & M \\
\hline Glycyrrhiza glabra L. & ES, M, IT & Humid habitats & $\begin{array}{l}\text { Mediterranean woodlands } \\
\text { and shrublands, } \\
\text { semi-steppe shrublands }\end{array}$ & M \\
\hline $\begin{array}{l}\text { Acacia saligna (Labill) H. L. } \\
\text { Wendl. Fil. (Acacia } \\
\text { cyanophylla Lindl.) }\end{array}$ & Australian & Light soils & $\begin{array}{l}\text { Mediterranean woodlands } \\
\text { and shrublands }\end{array}$ & $\mathrm{M}, \mathrm{Tr}$ \\
\hline $\begin{array}{l}\text { Calicotome villosa } \\
\text { (Poir.) Link }\end{array}$ & M & $\begin{array}{l}\text { Batha, phrygana, } \\
\text { Mediterranean maquis } \\
\text { and forests }\end{array}$ & $\begin{array}{l}\text { Mt. Hermon, Mediterranean } \\
\text { woodlands and shrublands, } \\
\text { semi-steppe shrublands } \\
\text { Deserts, Mediterranean }\end{array}$ & M \\
\hline $\begin{array}{l}\text { Retama raetam (Forssk.) } \\
\text { Webb. and Berthel. }\end{array}$ & SA & Sand & $\begin{array}{l}\text { woodlands and shrublands, } \\
\text { shrub-steppes, semi-steppe } \\
\text { shrublands }\end{array}$ & $\mathrm{M}, \mathrm{Tr}, \mathrm{SD}, \mathrm{ED}$ \\
\hline $\begin{array}{l}\text { Genista monspessulana (L.) } \\
\text { O. Bolós and Vigo. }\end{array}$ & M & $\begin{array}{l}\text { Batha, phrygana, } \\
\text { Mediterranean maquis } \\
\text { and forests }\end{array}$ & $\begin{array}{l}\text { Mt. Hermon, Mediterranean } \\
\text { woodlands and shrublands, } \\
\text { semi-steppe shrublands } \\
\text { Deserts, Mediterranean }\end{array}$ & M \\
\hline Acacia radiana Savi. & $S$ & $\begin{array}{l}\text { Deserts, thermophilous } \\
\text { plants }\end{array}$ & $\begin{array}{l}\text { woodlands and shrublands, } \\
\text { shrub-steppes, semi-steppe } \\
\text { shrublands }\end{array}$ & DX \\
\hline Ficus microcarpa L. F. & M, IT & Humid & $\begin{array}{l}\text { Mediterranean woodlands and } \\
\text { shrublands, semi-steppe } \\
\text { shrublands, shrub-steppes }\end{array}$ & M \\
\hline Ficus sycomorus L. & $S$ & Light soils & $\begin{array}{l}\text { Mediterranean woodlands and } \\
\text { shrublands, semi-steppe } \\
\text { shrublands, shrub-steppes }\end{array}$ & M \\
\hline Ficus cariaca L. & M, IT & Humid & $\begin{array}{c}\text { Deserts, Mediterranean } \\
\text { woodlands and shrublands, } \\
\text { shrub-steppes, semi-steppe } \\
\text { shrublands }\end{array}$ & $\mathrm{M}, \mathrm{Tr}, \mathrm{SD}$ \\
\hline Morus alba L. & M, IT, EC & Disturbed habitats & $\begin{array}{l}\text { Mediterranean woodlands } \\
\text { and shrublands }\end{array}$ & M \\
\hline Morus nigara L. & M, IT, EC & Disturbed habitats & $\begin{array}{l}\text { Mediterranean woodlands } \\
\text { and shrublands }\end{array}$ & M \\
\hline $\begin{array}{c}\text { Prosopis farcta } \\
\text { (Banks et Sol.) Macbride }\end{array}$ & IT & Batha, phrygana & $\begin{array}{l}\text { Deserts, semi-steppe } \\
\text { shrublands, shrub-steppes, } \\
\text { Mediterranean woodlands and } \\
\text { shrublands, Mt. Hermon }\end{array}$ & $\mathrm{M}, \mathrm{Tr}, \mathrm{SD}, \mathrm{ED}$ \\
\hline $\begin{array}{l}\text { Olea europaea L. (Olea sativa } \\
\text { Hoffmanns. and Link.) }\end{array}$ & M & $\begin{array}{l}\text { Mediterranean maquis } \\
\text { and forests, hard } \\
\text { rock outcrops }\end{array}$ & $\begin{array}{c}\text { Mediterranean woodlands and } \\
\text { shrublands, Mt. Hermon, } \\
\text { semi-steppe shrublands }\end{array}$ & M \\
\hline $\begin{array}{l}\text { Olea oleaster Hoffmanns. } \\
\text { and Link. (Olea sativa } \\
\text { Hoffmanns. and Link.) }\end{array}$ & M & $\begin{array}{l}\text { Mediterranean maquis } \\
\text { and forests, hard } \\
\text { rock outcrops }\end{array}$ & $\begin{array}{l}\text { Mediterranean woodlands and } \\
\text { shrublands, semi-steppe } \\
\text { shrublands }\end{array}$ & M \\
\hline Olea europaea var. sylvestris & M & $\begin{array}{l}\text { Mediterranean maquis } \\
\text { and forests, hard } \\
\text { rock outcrops }\end{array}$ & $\begin{array}{c}\text { Mediterranean woodlands and } \\
\text { shrublands, semi-steppe } \\
\text { shrublands }\end{array}$ & M \\
\hline $\begin{array}{c}\text { Phillyrea latifolia L. (Phillyria } \\
\text { media L.) }\end{array}$ & M & $\begin{array}{l}\text { Mediterranean maquis } \\
\text { and forests, hard } \\
\text { rock outcrops }\end{array}$ & $\begin{array}{l}\text { Mediterranean woodlands and } \\
\text { shrublands, semi-steppe } \\
\text { shrublands }\end{array}$ & M \\
\hline Salix alba $\mathrm{L}$. & ES, M, IT & Humid & $\begin{array}{l}\text { Mediterranean woodlands } \\
\text { and shrublands }\end{array}$ & M \\
\hline
\end{tabular}


Table 4. Cont.

\begin{tabular}{|c|c|c|c|c|}
\hline Species & Chorotype & Habitat & Distribution & Climate \\
\hline Populus alba L. & M & Humid & $\begin{array}{l}\text { Mediterranean woodlands and } \\
\text { shrublands, montane } \\
\text { vegetation of Mt. Hermon }\end{array}$ & M \\
\hline Populus euphratica Olivier & IT, SA & Humid & $\begin{array}{l}\text { Deserts, semi-steppe } \\
\text { shrublands, shrub-steppes, } \\
\text { Mediterranean woodlands } \\
\text { and shrubland }\end{array}$ & $\mathrm{M}, \mathrm{Tr}, \mathrm{SD}, \mathrm{ED}$ \\
\hline $\begin{array}{l}\text { Tamarix aphylla (L.) Karsten } \\
\text { (Tamarix articulate Vahl.) }\end{array}$ & S & $\begin{array}{l}\text { Desert, thermophilous } \\
\text { plants }\end{array}$ & $\begin{array}{l}\text { Mediterranean woodlands and } \\
\text { shrublands, semi-steppe } \\
\text { shrublands, shrub-steppes, } \\
\text { deserts and extreme deserts }\end{array}$ & ED \\
\hline Tamarix jordanis Boiss. & M & Humid & $\begin{array}{l}\text { Mediterranean woodlands and } \\
\text { shrublands, semi-steppe } \\
\text { shrublands, shrub-steppes, } \\
\text { deserts and extreme deserts }\end{array}$ & M, Tr, SD, ED \\
\hline Tamarix palaestina Bertol. & SA & $\begin{array}{l}\text { Deserts, thermophilous } \\
\text { plants }\end{array}$ & $\begin{array}{c}\text { Deserts, semi-steppe } \\
\text { shrublands, shrub-steppes, } \\
\text { Mediterranean woodlands } \\
\text { and shrublands }\end{array}$ & $\mathrm{Tr}, \mathrm{SD}, \mathrm{ED}$ \\
\hline $\begin{array}{c}\text { Tamarix nilotica } \\
\text { (Ehrenb.) Bunge } \\
\text { (Tamarix senegalensis DC.) }\end{array}$ & SA & Deserts, salty habitats & $\begin{array}{c}\text { Deserts, semi-steppe } \\
\text { shrublands, shrub-steppes, } \\
\text { Mediterranean woodlands } \\
\text { and shrublands }\end{array}$ & Tr, SD, ED \\
\hline Tamarix negevensis Zohary & SA & $\begin{array}{l}\text { Deserts, salty habitats, } \\
\text { thermophilous plants }\end{array}$ & Deserts & Tr, SD, ED \\
\hline Tamarix parviflora DC. & M & Deserts, salty habitats & $\begin{array}{l}\text { Mediterranean woodlands } \\
\text { and shrublands }\end{array}$ & M \\
\hline Tamarix tetragyna Ehrenb. & M, SA & $\begin{array}{l}\text { Saline sandy soils, } \\
\text { swamps, edges of } \\
\text { salt marshes }\end{array}$ & $\begin{array}{l}\text { Mediterranean woodlands and } \\
\text { shrublands, semi-steppe } \\
\text { shrublands, shrub-steppes, } \\
\text { deserts and extreme deserts }\end{array}$ & $\mathrm{M}, \mathrm{Tr}, \mathrm{SD}, \mathrm{ED}$ \\
\hline $\begin{array}{c}\text { Tamarix gennessarensis } \\
\text { Zohary }\end{array}$ & M & Humid & $\begin{array}{l}\text { Mediterranean woodlands and } \\
\text { shrublands, Mt. Hermon }\end{array}$ & M \\
\hline $\begin{array}{l}\text { Reaumuria negevensis } \\
\text { Zohary and Danin }\end{array}$ & SA & Shrub-steppes & $\begin{array}{l}\text { Deserts, shrub-steppes, } \\
\text { semi-steppe shrublands }\end{array}$ & $\mathrm{SD}, \mathrm{ED}$ \\
\hline Tamarix passerinoides Delile & S, SA & Deserts, salty habitats & Deserts & ED \\
\hline $\begin{array}{l}\text { Tamarix amplexicaulis } \\
\text { Ehrenb. }\end{array}$ & $\mathrm{S}, \mathrm{SA}$ & Deserts, salty habitats & Deserts & ED \\
\hline Acer obtusifolium Sm. & M & $\begin{array}{l}\text { Mediterranean maquis } \\
\text { and forests }\end{array}$ & Deserts & M \\
\hline $\begin{array}{l}\text { Acer monspessulanum L. } \\
\text { (Acer hermoneum (Bornm.) } \\
\text { Bornm. and Schweinf.) }\end{array}$ & M, ES & $\begin{array}{l}\text { Tragacanth shrub } \\
\text { vegetation } \\
\text { (Oro-Mediterranean) }\end{array}$ & Mt. Hermon & M \\
\hline $\begin{array}{c}\text { Polygonum palaestinum } \\
\text { Zohary }\end{array}$ & M, SA & Sand & $\begin{array}{l}\text { Mediterranean woodlands } \\
\text { and shrublands }\end{array}$ & $\mathrm{M}, \mathrm{Tr}, \mathrm{SD}$ \\
\hline $\begin{array}{l}\text { Persicaria lanigera } \\
\text { (R.Br.) Sojak }\end{array}$ & $\mathrm{T}$ & Humid & $\begin{array}{l}\text { Mediterranean woodlands } \\
\text { and shrublands }\end{array}$ & $\mathrm{M}, \mathrm{Tr}, \mathrm{SD}, \mathrm{ED}$ \\
\hline Atraphaxis spinosa L. & IT & Shrub-steppes & Deserts, shrub-steppes & $\mathrm{SD}, \mathrm{ED}$ \\
\hline $\begin{array}{l}\text { Rheum palaestinum } \\
\text { Feinbrun }\end{array}$ & IT & Shrub-steppes & Deserts, shrub-steppes & SD, ED \\
\hline Anagyris foetida $\mathrm{L}$. & M, IT & $\begin{array}{l}\text { Mediterranean maquis } \\
\text { and forests }\end{array}$ & $\begin{array}{l}\text { Semi-steppe shrublands, } \\
\text { Mediterranean woodlands } \\
\text { and shrublands }\end{array}$ & $\mathrm{M}, \mathrm{Tr}$ \\
\hline $\begin{array}{l}\text { Colutea abyssinica } \\
\text { Kunth and Bouche }\end{array}$ & IT & $\begin{array}{l}\text { Deserts, thermophilous } \\
\text { plants }\end{array}$ & $\begin{array}{l}\text { Deserts, Mediterranean } \\
\text { woodlands and shrublands, } \\
\text { semi-steppe shrublands }\end{array}$ & SD \\
\hline $\begin{array}{c}\text { Lycium barbarum } \\
\text { sensu Boiss. non L. } \\
\text { (Lycium depressum Stocks) }\end{array}$ & IT & $\begin{array}{l}\text { Deserts, thermophilous } \\
\text { plants }\end{array}$ & $\begin{array}{l}\text { Deserts, Mediterranean } \\
\text { woodlands and shrublands, } \\
\text { semi-steppe shrublands }\end{array}$ & ED \\
\hline
\end{tabular}


Table 4. Cont.

\begin{tabular}{|c|c|c|c|c|}
\hline Species & Chorotype & Habitat & Distribution & Climate \\
\hline $\begin{array}{l}\text { Lycium europaeum } \mathrm{L} . \\
\text { (Lycium mediterraneum } \\
\text { Dunal.) }\end{array}$ & M & $\begin{array}{l}\text { Mediterranean maquis } \\
\text { and forests, } \\
\text { shrub-steppes }\end{array}$ & $\begin{array}{c}\text { Mediterranean woodlands and } \\
\text { shrublands, semi-steppe } \\
\text { shrublands, shrub-steppes, } \\
\text { deserts and extreme deserts }\end{array}$ & $\mathrm{M}, \mathrm{Tr}$ \\
\hline Nicotiana glauca Graham & PT & Disturbed habitats & $\begin{array}{c}\text { Mediterranean woodlands and } \\
\text { shrublands, semi-steppe } \\
\text { shrublands, shrub-steppes, } \\
\text { deserts and extreme deserts }\end{array}$ & SD, ED \\
\hline $\begin{array}{l}\text { Lycium schweinfurthii } \\
\text { Dammer }\end{array}$ & M & Light soils & $\begin{array}{l}\text { Deserts, Mediterranean } \\
\text { woodlands and shrublands, } \\
\text { semi-steppe shrublands }\end{array}$ & M \\
\hline $\begin{array}{c}\text { Lycium shawii } \\
\text { Roem. and Schult. } \\
\text { (Lycium arabicum Boiss.) }\end{array}$ & SA, S & $\begin{array}{l}\text { Deserts, thermophilous } \\
\text { plants }\end{array}$ & $\begin{array}{c}\text { Deserts, semi-steppe } \\
\text { shrublands, shrub-steppes, } \\
\text { Mediterranean woodlands } \\
\text { and shrublands }\end{array}$ & SD, ED \\
\hline Solanum sinaicum Boiss. & ES, M, IT & $\begin{array}{c}\text { Disturbed habitats, } \\
\text { cultivated areas (weeds) }\end{array}$ & $\begin{array}{c}\text { Deserts, semi-steppe } \\
\text { shrublands, shrub-steppes, } \\
\text { Mediterranean woodlands } \\
\text { and shrublands }\end{array}$ & Tr, SD, ED \\
\hline Solanum incanum L. & $S$ & $\begin{array}{l}\text { Deserts, thermophilous } \\
\text { plants }\end{array}$ & $\begin{array}{c}\text { Deserts, semi-steppe } \\
\text { shrublands, shrub-steppes, } \\
\text { Mediterranean woodlands } \\
\text { and shrublands }\end{array}$ & ED \\
\hline $\begin{array}{l}\text { Phlomis pungens Willd. } \\
\text { (Phlomis herba-venti L.) }\end{array}$ & M, IT & $\begin{array}{l}\text { Batha, phrygana, } \\
\text { cultivated areas }\end{array}$ & $\begin{array}{l}\text { Mediterranean woodlands } \\
\text { and shrublands }\end{array}$ & M \\
\hline $\begin{array}{l}\text { Phlomis brachyodon (Boiss.) } \\
\text { Zohary ex. Rech. F. }\end{array}$ & IT & $\begin{array}{c}\text { Batha, phrygana, } \\
\text { cultivated areas (weeds) }\end{array}$ & $\begin{array}{l}\text { Mediterranean woodlands } \\
\text { and shrublands }\end{array}$ & $\mathrm{M}, \mathrm{Tr}$ \\
\hline Phlomis chrysophylla Boiss. & M & Batha, phrygana & $\begin{array}{l}\text { Mediterranean woodlands and } \\
\text { shrublands, Mt. Hermon }\end{array}$ & M \\
\hline $\begin{array}{l}\text { Ballota saxatilis } \\
\text { Sieber ex. C. Presl. }\end{array}$ & M & Hard rock outcrops & $\begin{array}{l}\text { Mediterranean woodlands and } \\
\text { shrublands, semi-steppe } \\
\text { shrublands, Mt. Hermon }\end{array}$ & M \\
\hline Ballota philistaea Bornm. & M & Sand & $\begin{array}{l}\text { Mediterranean woodlands } \\
\text { and shrublands }\end{array}$ & M \\
\hline $\begin{array}{l}\text { Ballota undulata (Sieber ex } \\
\text { Fresen.) Bentham }\end{array}$ & M & $\begin{array}{l}\text { Batha, phrygana, hard } \\
\text { rock outcrops }\end{array}$ & $\begin{array}{c}\text { Shrub-steppes, Mediterranean } \\
\text { woodlands and shrublands, } \\
\text { deserts, semi-steppe } \\
\text { shrublands, Mt. Hermon }\end{array}$ & $\mathrm{M}, \mathrm{Tr}, \mathrm{SD}$ \\
\hline $\begin{array}{c}\text { Bassia arabica (Boiss.) } \\
\text { Maireand Weiller } \\
\text { (Chenolea arabica Boiss.) }\end{array}$ & SA & Sandy soils & $\begin{array}{c}\text { Deserts, semi-steppe } \\
\text { shrublands, shrub-steppes }\end{array}$ & SD, ED \\
\hline Phlomis platystegia Post. & IT & Batha, phrygana & $\begin{array}{l}\text { Mediterranean woodlands } \\
\text { and shrublands, } \\
\text { semi-steppe shrublands }\end{array}$ & SD \\
\hline Phlomis viscosa Poiret. & M & Batha, phrygana & $\begin{array}{c}\text { Mediterranean woodlands and } \\
\text { shrublands, montane } \\
\text { vegetation of Mt. Hermon } \\
\text { Semi-steppe shrublands, }\end{array}$ & M \\
\hline Salvia eigii Zohay & M & Batha, phrygana & $\begin{array}{l}\text { Mediterranean woodlands } \\
\text { and shrublands }\end{array}$ & M \\
\hline Salvia dominica L. & M & Batha, phrygana & $\begin{array}{l}\text { Semi-steppe shrublands, } \\
\text { Mediterranean woodlands and } \\
\text { shrublands and deserts }\end{array}$ & $\mathrm{M}, \mathrm{Tr}$ \\
\hline Salvia lanigera Poir. & M, SA & Shrub-steppes & $\begin{array}{c}\text { Deserts, semi-steppe } \\
\text { shrublands, Mediterranean } \\
\text { woodlands and shrublands, } \\
\text { shrub-steppes }\end{array}$ & M, SD \\
\hline
\end{tabular}


Table 4. Cont.

\begin{tabular}{|c|c|c|c|c|}
\hline Species & Chorotype & Habitat & Distribution & Climate \\
\hline Satureja thymbra L. & M & Batha, phrygana & $\begin{array}{l}\text { Mediterranean woodlands } \\
\text { and shrublands }\end{array}$ & M \\
\hline $\begin{array}{l}\text { Satureja thymbrifolia } \\
\text { Hedge and Feinbrun }\end{array}$ & IT, SA & Batha, phrygana & $\begin{array}{l}\text { Mediterranean woodlands } \\
\text { and shrublands }\end{array}$ & SD \\
\hline Stachys palaestina $\mathrm{L}$. & M & Hard rock outcrops & $\begin{array}{l}\text { Mediterranean woodlands and } \\
\text { shrublands, semi-steppe } \\
\text { shrublands, montane } \\
\text { vegetation of Mt. Hermon }\end{array}$ & M \\
\hline $\begin{array}{l}\text { Salvia fruticosa Miller } \\
\text { (Salvia triloba L. f.) }\end{array}$ & M & $\begin{array}{l}\text { Mediterranean maquis } \\
\text { and forests }\end{array}$ & $\begin{array}{l}\text { Mediterranean woodlands } \\
\text { and shrublands }\end{array}$ & M \\
\hline Salvia officinalis L. & M & $\begin{array}{l}\text { Mediterranean maquis } \\
\text { and forests }\end{array}$ & $\begin{array}{l}\text { Mediterranean woodlands } \\
\text { and shrublands }\end{array}$ & M \\
\hline Salvia aegyptiaca L. & SA & $\begin{array}{c}\text { Batha, phrygana, } \\
\text { disturbed habitats, } \\
\text { cultivated areas (weeds) }\end{array}$ & $\begin{array}{l}\text { Semi-steppe shrublands, } \\
\text { Mediterranean woodlands and } \\
\text { shrublands, Mt. Hermon } \\
\text { Semi-steppe shrublands, }\end{array}$ & $\mathrm{SD}, \mathrm{ED}$ \\
\hline Salvia palaestina Benth. & M, IT & Batha, phrygana & $\begin{array}{l}\text { Mediterranean woodlands } \\
\text { and shrublands }\end{array}$ & $\mathrm{M}, \mathrm{Tr}, \mathrm{SD}$ \\
\hline Salvia sclarea L. & M, IT & Batha, phrygana & $\begin{array}{l}\text { Mediterranean woodlands } \\
\text { and shrublands }\end{array}$ & M \\
\hline $\begin{array}{c}\text { Micromeria fruticosa (L.) } \\
\text { Druce. (Micromeria } \\
\text { serpyllifolia } \\
\text { (M. Bieb.) Boiss.) }\end{array}$ & M & Hard rock outcrops & $\begin{array}{l}\text { Semi-steppe shrublands, } \\
\text { Mediterranean woodlands } \\
\text { and shrublands }\end{array}$ & $\mathrm{M}, \mathrm{Tr}$ \\
\hline Teucrium capitatum $\mathrm{L}$. & M, IT & Batha, phrygana & $\begin{array}{l}\text { Semi-steppe shrublands, Mt. } \\
\text { Hermon, deserts, shrub-steppes, } \\
\text { Mediterranean woodlands } \\
\text { and shrublands }\end{array}$ & $\mathrm{M}, \mathrm{TZ}, \mathrm{SD}$ \\
\hline Teucrium creticum $\mathrm{L}$. & M & $\begin{array}{l}\text { Mediterranean maquis } \\
\text { and forests }\end{array}$ & $\begin{array}{l}\text { Mediterranean woodlands } \\
\text { and shrublands }\end{array}$ & M \\
\hline $\begin{array}{l}\text { Thymbra capitata (L.) Cav. } \\
\text { (Coridothymus capitatus } \\
\text { (L.) Rchb.f.) }\end{array}$ & M & Batha, phrygana & $\begin{array}{l}\text { Mt. Hermon, semi-steppe } \\
\text { shrublands, Mediterranean } \\
\text { woodlands and shrublands }\end{array}$ & M \\
\hline Thymbra spicata L. & M & Batha, phrygana & $\begin{array}{l}\text { Mediterranean woodlands } \\
\text { and shrublands }\end{array}$ & M \\
\hline $\begin{array}{l}\text { Jacaranda acutifolia Humb. } \\
\text { and Bonpl. (Jacaranda } \\
\text { mimosaefolia D. Don.) }\end{array}$ & M & Batha, phrygana & $\begin{array}{l}\text { Mediterranean woodlands } \\
\text { and shrublands }\end{array}$ & M \\
\hline Capparis spinosa $\mathrm{L}$. & M & Heavy soils & $\begin{array}{c}\text { Mediterranean woodlands and } \\
\text { shrublands, semi-steppe } \\
\text { shrublands, shrub-steppes, } \\
\text { montane vegetation of } \\
\text { Mt. Hermon }\end{array}$ & $\mathrm{M}, \mathrm{Tr}, \mathrm{SD}$ \\
\hline Capparis sicula Duh. & M, IT, SA & Disturbed habitats & $\begin{array}{l}\text { Semi-steppe shrublands, } \\
\text { Mediterranean woodlands } \\
\text { and shrublands }\end{array}$ & $\mathrm{M}, \mathrm{Tr}$ \\
\hline Capparis aegyptia Lam. & IT, SA & Heavy soils & $\begin{array}{c}\text { Semi-steppe shrublands, } \\
\text { shrub-steppes, deserts and } \\
\text { extreme deserts }\end{array}$ & $\mathrm{SD}, \mathrm{ED}$ \\
\hline Celtis australis L. & M & $\begin{array}{l}\text { Mediterranean maquis } \\
\text { and forests }\end{array}$ & $\begin{array}{l}\text { Mediterranean woodlands and } \\
\text { shrublands, montane } \\
\text { vegetation of Mt. Hermon }\end{array}$ & M \\
\hline Atriplex halimus L. & M, SA & Salty habitats & $\begin{array}{l}\text { Shrub-steppes, semi-steppe } \\
\text { shrublands, deserts, } \\
\text { Mediterranean woodlands } \\
\text { and shrublands }\end{array}$ & $\mathrm{Tr}, \mathrm{SD}, \mathrm{ED}$ \\
\hline
\end{tabular}


Table 4. Cont.

\begin{tabular}{|c|c|c|c|c|}
\hline Species & Chorotype & Habitat & Distribution & Climate \\
\hline $\begin{array}{l}\text { Noaea mucronata (Forssk.) } \\
\text { Asch. and Schweinf. }\end{array}$ & IT & Shrub-steppes & $\begin{array}{c}\text { Deserts, shrub-steppes, } \\
\text { semi-steppe shrublands, } \\
\text { Mediterranean woodlands and } \\
\text { shrublands, Mt. Hermon }\end{array}$ & $\mathrm{Tr}, \mathrm{SD}, \mathrm{ED}$ \\
\hline $\begin{array}{l}\text { Haloxylon persicum Bunge } \\
\text { Haloxylon negevensis }\end{array}$ & IT & Sand & Deserts & ED \\
\hline $\begin{array}{l}\text { (Iljin and Zohary) L. Boulos } \\
\text { (Hammada negevensis } \\
\text { Iljin and Zohary) }\end{array}$ & SA & $\begin{array}{l}\text { Sand, deserts, } \\
\text { shrub-steppes }\end{array}$ & $\begin{array}{l}\text { Shrub-steppes, semi-steppe } \\
\text { shrublands, deserts }\end{array}$ & SD, ED \\
\hline Salicornia fruticosa (L.) L. & M, SA & $\begin{array}{l}\text { Humid habitats, } \\
\text { salty habitats }\end{array}$ & $\begin{array}{l}\text { Shrub-steppes, deserts, } \\
\text { semi-steppe shrublands, } \\
\text { Mediterranean woodlands } \\
\text { and shrubland }\end{array}$ & $\mathrm{M}, \mathrm{SD}, \mathrm{ED}$ \\
\hline $\begin{array}{l}\text { Aerva javanica (Burm.f.) } \\
\text { Juss. ex. Schult. }\end{array}$ & $\mathrm{T}$ & $\begin{array}{l}\text { Deserts, thermophilous } \\
\text { (heat-loving) plants }\end{array}$ & $\begin{array}{l}\text { Semi-steppe shrublands, } \\
\text { shrub-steppes, deserts and } \\
\text { extreme deserts }\end{array}$ & $\mathrm{SD}, \mathrm{ED}$ \\
\hline Salsola imbricata Forssk. & $S$ & $\begin{array}{l}\text { Desert, thermophilous } \\
\text { plants }\end{array}$ & $\begin{array}{l}\text { shrub-steppes, semi-steppe } \\
\text { shrublands, Mediterranean } \\
\text { woodlands and } \\
\text { shrublands, deserts }\end{array}$ & DX \\
\hline Salsola cyclophylla Baker & SA & $\begin{array}{l}\text { Deserts, salty habitats, } \\
\text { thermophilous plants }\end{array}$ & Shrub-steppes, deserts & $\mathrm{SD}, \mathrm{ED}$ \\
\hline $\begin{array}{l}\text { Arthrocaulon macrostachyum } \\
\text { (Moric.) Piirainen } \\
\text { and G. Kadereit }\end{array}$ & M & $\begin{array}{l}\text { Humid habitats, } \\
\text { salty habitats }\end{array}$ & $\begin{array}{l}\text { Semi-steppe shrublands, } \\
\text { Mediterranean woodlands and } \\
\text { shrublands, deserts }\end{array}$ & $\mathrm{M}, \mathrm{Tr}, \mathrm{SD}, \mathrm{ED}$ \\
\hline $\begin{array}{l}\text { Suaeda palaestina } \\
\text { Eig. and Zohary }\end{array}$ & SA, S & Deserts, salty habitats & $\begin{array}{c}\text { Deserts, semi, } \\
\text { steppe shrublands }\end{array}$ & $\mathrm{SD}, \mathrm{ED}$ \\
\hline $\begin{array}{l}\text { Halothamnus lancifolius } \\
\text { (Boiss.) Kothe-Heinr. }\end{array}$ & IT & $\begin{array}{l}\text { Mediterranean maquis } \\
\text { and forests }\end{array}$ & $\begin{array}{l}\text { Deserts, shrub-steppes, } \\
\text { semi-steppe shrublands, } \\
\text { Mediterranean woodlands } \\
\text { and shrublands }\end{array}$ & $\mathrm{M}, \mathrm{Tr}, \mathrm{SD}, \mathrm{DX}$ \\
\hline $\begin{array}{l}\text { Haloxylon salicornicum } \\
\text { (Moq.) Bunge ex Boiss. }\end{array}$ & $S$ & Sand & $\begin{array}{l}\text { Deserts, shrub-steppes, } \\
\text { semi-steppe shrublands }\end{array}$ & DX \\
\hline Ononis natrix $\mathrm{L}$. & M & Batha, phrygana & $\begin{array}{l}\text { Mediterranean woodlands and } \\
\text { shrublands, semi-steppe } \\
\text { shrublands, Mt. Hermon }\end{array}$ & $\mathrm{M}, \mathrm{Tr}, \mathrm{SD}$ \\
\hline Arbutus andrachne L. & M & $\begin{array}{l}\text { Mediterranean maquis } \\
\text { and forests }\end{array}$ & $\begin{array}{l}\text { Mediterranean woodlands } \\
\text { and shrublands, } \\
\text { semi-steppe shrublands }\end{array}$ & M \\
\hline Laurus nobilis L. & M & $\begin{array}{l}\text { Mediterranean maquis } \\
\text { and forests }\end{array}$ & $\begin{array}{l}\text { Mediterranean woodlands and } \\
\text { shrublands, Mt. Hermon }\end{array}$ & M \\
\hline $\begin{array}{l}\text { Pteridium aquilinum } \\
\text { (L.) Kuhn }\end{array}$ & PT & Humid & $\begin{array}{l}\text { Mediterranean woodlands } \\
\text { and shrublands }\end{array}$ & M \\
\hline Melia azedarach $\mathrm{L}$. & M & Disturbed habitats & $\begin{array}{l}\text { Mediterranean woodlands } \\
\text { and shrublands, } \\
\text { semi-steppe shrublands }\end{array}$ & M \\
\hline Paeonia mascula (L.) Miller & M, ES & $\begin{array}{l}\text { Mediterranean maquis } \\
\text { and forests }\end{array}$ & $\begin{array}{l}\text { Mediterranean woodlands } \\
\text { and shrublands }\end{array}$ & M \\
\hline Azolla filiculoides Lam. & A & Humid habitats & $\begin{array}{l}\text { Mediterranean woodlands } \\
\text { and shrublands }\end{array}$ & M \\
\hline $\begin{array}{l}\text { Leptadenia pyrotechnica } \\
\text { (Forssk.) Decne. }\end{array}$ & SA, S & $\begin{array}{l}\text { Deserts, thermophilous } \\
\text { plants }\end{array}$ & Deserts & ED \\
\hline Styrex officinalis L. & M & $\begin{array}{l}\text { Mediterranean maquis } \\
\text { and forests }\end{array}$ & $\begin{array}{l}\text { Mt. Hermon, Mediterranean } \\
\text { woodlands and shrublands, } \\
\text { semi-steppe shrublands } \\
\text { Semi-steppe shrublands, }\end{array}$ & M \\
\hline Achillea aleppica DC. & IT & Batha, phrygana & $\begin{array}{l}\text { Mediterranean woodlands } \\
\text { and shrublands }\end{array}$ & $\mathrm{TZ}$ \\
\hline
\end{tabular}


Table 4. Cont.

\begin{tabular}{|c|c|c|c|c|}
\hline Species & Chorotype & Habitat & Distribution & Climate \\
\hline $\begin{array}{l}\text { Achillea fragrantissima } \\
\text { (Forssk.) Sch. Bip. }\end{array}$ & IT, SA & Shrub-steppes & $\begin{array}{c}\text { Shrub-steppes, Mediterranean } \\
\text { woodlands and shrublands, } \\
\text { deserts, semi-steppe } \\
\text { shrublands, Mt. Hermon }\end{array}$ & SD, ED \\
\hline Gundelia tournefortii $\mathrm{L}$. & IT & $\begin{array}{l}\text { Batha, phrygana, } \\
\text { Shrub-steppes }\end{array}$ & $\begin{array}{l}\text { Mt. Hermon, Mediterranean } \\
\text { woodlands and shrublands, } \\
\text { semi-steppe shrublands }\end{array}$ & $\mathrm{M}, \mathrm{Tr}, \mathrm{SD}$ \\
\hline Artemisia arborescens L. & M & $\begin{array}{l}\text { Mediterranean maquis } \\
\text { and forest }\end{array}$ & $\begin{array}{l}\text { Mediterranean woodlands } \\
\text { and shrublands }\end{array}$ & $\mathrm{M}, \mathrm{TZ}$ \\
\hline Artemisia monosperma Delile & SA & Sand & $\begin{array}{l}\text { Mediterranean woodlands and } \\
\text { shrublands, semi-steppe } \\
\text { shrublands, shrub-steppes, } \\
\text { deserts and extreme deserts }\end{array}$ & $\mathrm{M}, \mathrm{Tr}, \mathrm{SD}, \mathrm{ED}$ \\
\hline $\begin{array}{l}\text { Anvillea garcinii } \\
\text { (Burm.f.) DC. }\end{array}$ & SA & $\begin{array}{l}\text { Deserts, thermophilous } \\
\text { plants }\end{array}$ & Deserts & $\mathrm{SD}, \mathrm{ED}$ \\
\hline Pluchea dioscoridis (L.) DC. & SA, S & Humid & $\begin{array}{l}\text { Mediterranean woodlands and } \\
\text { shrublands, deserts, } \\
\text { semi-steppe shrublands }\end{array}$ & $\mathrm{M}, \mathrm{Tr}, \mathrm{SD}$ \\
\hline Artemisia sieberi Besser & IT & Shrub-steppes & $\begin{array}{l}\text { Deserts, shrub-steppes, } \\
\text { semi-steppe shrublands, } \\
\text { Mediterranean woodlands } \\
\text { and shrublands }\end{array}$ & SD, ED \\
\hline $\begin{array}{l}\text { Asparagus horridus L. } \\
\text { (Asparagus stipularis } \\
\text { Forssk.) }\end{array}$ & M, SA & $\begin{array}{l}\text { Sand, hard } \\
\text { rock outcrops }\end{array}$ & $\begin{array}{l}\text { Shrub-steppes, Mediterranean } \\
\text { woodlands and shrublands, } \\
\text { deserts, semi-steppe shrublands }\end{array}$ & $\mathrm{M}, \mathrm{Tr}, \mathrm{SD}, \mathrm{ED}$ \\
\hline Asparagus palaestinus Baker & M & $\begin{array}{l}\text { Mediterranean maquis } \\
\text { and forests }\end{array}$ & $\begin{array}{l}\text { Mediterranean woodlands } \\
\text { and shrublands }\end{array}$ & $\mathrm{M}, \mathrm{Tr}$ \\
\hline Smilax aspera L. & M & $\begin{array}{l}\text { Mediterranean maquis } \\
\text { and forests }\end{array}$ & $\begin{array}{l}\text { Mediterranean woodlands } \\
\text { and shrublands }\end{array}$ & M \\
\hline Parkinsonia aculeata L. & $\mathrm{AM}$ & Disturbed habitats & $\begin{array}{l}\text { Deserts, shrub-steppes, } \\
\text { semi-steppe shrublands, } \\
\text { Mediterranean woodlands } \\
\text { and shrublands }\end{array}$ & $\mathrm{M}, \mathrm{Tr}$ \\
\hline Ochradenus baccatus Delile & SUA & $\begin{array}{l}\text { Deserts, thermophilous } \\
\text { plants }\end{array}$ & $\begin{array}{l}\text { Deserts, shrub-steppes, } \\
\text { semi-steppe shrublands, } \\
\text { Mediterranean woodlands } \\
\text { and shrublands }\end{array}$ & $\mathrm{SD}, \mathrm{ED}$ \\
\hline Nerium oleander L. & M & Humid & $\begin{array}{l}\text { Deserts, shrub-steppes, } \\
\text { semi-steppe shrublands, } \\
\text { Mediterranean woodlands } \\
\text { and shrublands }\end{array}$ & $\mathrm{M}, \mathrm{Tr}, \mathrm{SD}$ \\
\hline Cynanchum acutum L. & M, IT & Humid & $\begin{array}{l}\text { Deserts, shrub-steppes, } \\
\text { semi-steppe shrublands, } \\
\text { Mediterranean woodlands } \\
\text { and shrublands }\end{array}$ & $\mathrm{M}, \mathrm{Tr}$ \\
\hline Periploca aphylla Decne. & $S$ & Hard rock outcrops & $\begin{array}{l}\text { Deserts, shrub-steppes, } \\
\text { semi-steppe shrublands, } \\
\text { Mediterranean woodlands } \\
\text { and shrublands }\end{array}$ & SD, ED \\
\hline $\begin{array}{l}\text { Moringa peregrina } \\
\text { (Forssk.) Fiori }\end{array}$ & A & $\begin{array}{l}\text { Deserts, thermophilous } \\
\text { plants }\end{array}$ & $\begin{array}{l}\text { Mediterranean woodlands and } \\
\text { shrublands, deserts, } \\
\text { semi-steppe shrublands }\end{array}$ & ED \\
\hline Grewia villosa Willd. & $\mathrm{T}$ & $\begin{array}{l}\text { Hard rock outcrops, } \\
\text { thermophilous plants }\end{array}$ & Deserts & ED \\
\hline Ephedra aphylla Forskal & SA & $\begin{array}{c}\text { Sand, hard rock } \\
\text { outcrops }\end{array}$ & $\begin{array}{l}\text { Deserts, shrub-steppes, } \\
\text { semi-steppe shrublands, } \\
\text { Mediterranean woodlands } \\
\text { and shrublands }\end{array}$ & $\mathrm{Tr}, \mathrm{SD}, \mathrm{ED}$ \\
\hline
\end{tabular}


Table 4. Cont.

\begin{tabular}{|c|c|c|c|c|}
\hline Species & Chorotype & Habitat & Distribution & Climate \\
\hline $\begin{array}{c}\text { Ephedra foeminea Forssk } \\
\text { (Ephedra campylopoda } \\
\text { C. A. Mey) }\end{array}$ & M & $\begin{array}{l}\text { Mediterranean maquis } \\
\text { and forests }\end{array}$ & $\begin{array}{l}\text { Mediterranean woodlands and } \\
\text { shrublands, semi-steppe } \\
\text { shrublands, shrub-steppes, } \\
\text { montane vegetation of } \\
\text { Mt. Hermon }\end{array}$ & $\mathrm{M}, \mathrm{Tr}, \mathrm{SD}$ \\
\hline Anabasis setifera Moq. & SA & Deserts & $\begin{array}{r}\text { Deserts, shrub-steppes, } \\
\text { semi-steppe shrublands }\end{array}$ & SD, ED \\
\hline Anabasis syriaca Iljin & IT & Shrub-steppes & $\begin{array}{l}\text { Deserts, shrub-steppes, } \\
\text { semi-steppe shrublands }\end{array}$ & $\mathrm{SD}, \mathrm{ED}$ \\
\hline Alkanna orientalis (L.) Boiss. & M, IT & Shrub-steppes & Mt. Hermon & M, SD \\
\hline $\begin{array}{l}\text { Alkanna strigosa } \\
\text { Boiss and Hohen. }\end{array}$ & M & Batha, phrygana & $\begin{array}{l}\text { Semi-steppe shrublands, } \\
\text { Mediterranean woodlands } \\
\text { and shrublands }\end{array}$ & $\mathrm{M}, \mathrm{Tr}$ \\
\hline Alkanna galilaea Boiss. & M & Batha, phrygana & $\begin{array}{l}\text { Mediterranean woodlands } \\
\text { and shrublands }\end{array}$ & M \\
\hline $\begin{array}{l}\text { Alkanna tinctoria } \\
\text { (L.) Tausch }\end{array}$ & M & Sand & $\begin{array}{l}\text { Mediterranean woodlands } \\
\text { and shrublands, } \\
\text { semi-steppe shrublands }\end{array}$ & $\mathrm{M}, \mathrm{Tr}$ \\
\hline $\begin{array}{l}\text { Ailanthus altissima } \\
\text { (Mill.) Swingle }\end{array}$ & $\mathrm{T}$ & Disturbed habitats & $\begin{array}{l}\text { Mediterranean woodlands } \\
\text { and shrublands }\end{array}$ & M \\
\hline Rubia tinctorum $\mathrm{L}$. & M, IT & $\begin{array}{l}\text { Mediterranean maquis } \\
\text { and forests }\end{array}$ & $\begin{array}{l}\text { Mediterranean woodlands } \\
\text { and shrublands, } \\
\text { semi-steppe shrublands }\end{array}$ & $\operatorname{Tr}$ \\
\hline Rubia tenuifolia D'Urv. & M & $\begin{array}{l}\text { Mediterranean maquis } \\
\text { and forests }\end{array}$ & $\begin{array}{l}\text { Mediterranean woodlands and } \\
\text { shrublands, semi-steppe } \\
\text { shrublands, Mt. Hermon }\end{array}$ & $\mathrm{M}, \mathrm{Tr}$ \\
\hline Ruta chalepensis L. & M & $\begin{array}{l}\text { Mediterranean maquis } \\
\text { and forests }\end{array}$ & $\begin{array}{l}\text { Mediterranean woodlands } \\
\text { and shrublands, } \\
\text { semi-steppe shrublands }\end{array}$ & M \\
\hline Thymelaea hirsuta (L.) Endl. & M, SA & Batha, phrygana & $\begin{array}{l}\text { Shrub-steppes, deserts, } \\
\text { semi-steppe shrublands, } \\
\text { Mediterranean woodlands } \\
\text { and shrublands }\end{array}$ & $\mathrm{M}, \mathrm{Tr}, \mathrm{SA}$ \\
\hline Verbascum tiberiadis Boiss. & M & Batha, phrygana & $\begin{array}{l}\text { Mediterranean woodlands } \\
\text { and shrublands, } \\
\text { semi-steppe shrublands }\end{array}$ & $\mathrm{M}, \mathrm{Tr}$ \\
\hline Verbascum sinaiticum Benth. & IT, SA & Deserts, shrub-steppes & $\begin{array}{l}\text { Deserts, shrub-steppes, } \\
\text { semi-steppe shrublands, } \\
\text { Mediterranean woodlands and } \\
\text { shrublands, Mt. Hermon }\end{array}$ & $\mathrm{Tr}, \mathrm{SD}$ \\
\hline Verbascum galilaeum Boiss. & M & Humid & $\begin{array}{l}\text { Mediterranean woodlands } \\
\text { and shrublands }\end{array}$ & M \\
\hline $\begin{array}{l}\text { Verbascum jordanicum } \\
\text { Murb. }\end{array}$ & IT & $\begin{array}{l}\text { Mediterranean maquis } \\
\text { and forests }\end{array}$ & $\begin{array}{l}\text { Mediterranean woodlands and } \\
\text { shrublands, semi-steppe } \\
\text { shrublands, shrub-steppes }\end{array}$ & $\mathrm{Tr}, \mathrm{SD}$ \\
\hline Verbascum eremobium Murb. & IT & Deserts, shrub-steppes & Deserts & $\mathrm{SD}, \mathrm{ED}$ \\
\hline $\begin{array}{l}\text { Verbascum fruticulosum } \\
\text { Post. }\end{array}$ & IT & Shrub-steppes & $\begin{array}{l}\text { Deserts, shrub-steppes, } \\
\text { semi-steppe shrublands }\end{array}$ & Tr, SD, ED \\
\hline Verbascum gaillardotii Boiss. & M & $\begin{array}{l}\text { Mediterranean maquis } \\
\text { and forests }\end{array}$ & $\begin{array}{l}\text { Mediterranean woodlands } \\
\text { and shrublands }\end{array}$ & M \\
\hline Verbena officinalis L. & Sub T & Humid & $\begin{array}{l}\text { Mediterranean woodlands and } \\
\text { shrublands, semi-steppe } \\
\text { shrublands, shrub-steppes, } \\
\text { deserts and extreme deserts, } \\
\text { montane vegetation of } \\
\text { Mt. Hermon }\end{array}$ & $\mathrm{M}, \mathrm{Tr}$ \\
\hline
\end{tabular}


Table 4. Cont.

\begin{tabular}{|c|c|c|c|c|}
\hline Species & Chorotype & Habitat & Distribution & Climate \\
\hline Hedera helix L. & M, ES & $\begin{array}{l}\text { Mediterranean maquis } \\
\text { and forests, hard } \\
\text { rock outcrops }\end{array}$ & $\begin{array}{l}\text { Mediterranean woodlands } \\
\text { and shrublands }\end{array}$ & M \\
\hline $\begin{array}{l}\text { Erodium crassifolium } \\
\text { L'Her. ex. Aiton. } \\
\text { (Erodium hirtum Willd.) }\end{array}$ & SA & Deserts, shrub-steppes & $\begin{array}{l}\text { Mediterranean woodlands and } \\
\text { shrublands, semi-steppe } \\
\text { shrublands, shrub-steppes, } \\
\text { deserts and extreme deserts, } \\
\text { montane vegetation of } \\
\text { Mt. Hermon }\end{array}$ & $\mathrm{SD}, \mathrm{ED}$ \\
\hline $\begin{array}{l}\text { Erodium glaucophyllum } \\
\text { (L.) L'Hér. }\end{array}$ & SA & Saline soils & $\begin{array}{r}\text { Deserts, shrub-steppes, } \\
\text { semi-steppe shrublands }\end{array}$ & $\mathrm{SD}, \mathrm{ED}$ \\
\hline $\begin{array}{l}\text { Erodium arborescens } \\
\text { (Desf.) Willd. }\end{array}$ & SA & Deserts, shrub-steppes & $\begin{array}{l}\text { Deserts, shrub-steppes, } \\
\text { semi-steppe shrublands }\end{array}$ & ED \\
\hline $\begin{array}{c}\text { Erodium acaule (L.) } \\
\text { Becherer and Thell. } \\
\text { (Erodium romanum (L.) Willd.) }\end{array}$ & M & Batha, phrygana & $\begin{array}{l}\text { Mt. Hermon, Mediterranean } \\
\text { woodlands and shrublands }\end{array}$ & M \\
\hline Eryngium creticum Lam. & M & Batha, phrygana & $\begin{array}{l}\text { Mediterranean woodlands and } \\
\text { shrublands, semi-steppe } \\
\text { shrublands, shrub-steppes, } \\
\text { deserts and extreme deserts, } \\
\text { montane vegetation of } \\
\text { Mt. Hermon }\end{array}$ & $\mathrm{M}, \mathrm{Tr}$ \\
\hline $\begin{array}{l}\text { Eryngium falcatum } \\
\text { F. Delaroche }\end{array}$ & M & $\begin{array}{l}\text { Mediterranean maquis } \\
\text { and forests, hard } \\
\text { rock outcrops }\end{array}$ & $\begin{array}{l}\text { Mediterranean woodlands and } \\
\text { shrublands, semi-steppe } \\
\text { shrublands, shrub-steppes, } \\
\text { deserts and extreme deserts, } \\
\text { montane vegetation of } \\
\text { Mt. Hermon }\end{array}$ & M \\
\hline Eryngium glomeratum Lam. & M & $\begin{array}{l}\text { Batha, phrygana, } \\
\text { tragacanth shrub } \\
\text { vegetation } \\
\text { (Oro-Mediterranean), } \\
\text { hard rock outcrops }\end{array}$ & $\begin{array}{c}\text { Mediterranean woodlands and } \\
\text { shrublands, semi-steppe } \\
\text { shrublands, shrub-steppes, } \\
\text { deserts and extreme deserts, } \\
\text { montane vegetation of } \\
\text { Mt. Hermon }\end{array}$ & $\mathrm{M}, \mathrm{Tr}, \mathrm{SD}$ \\
\hline Eryngium maritimum L. & M, SA, IT & Coastal & $\begin{array}{c}\text { Mediterranean woodlands and } \\
\text { shrublands, semi-steppe } \\
\text { shrublands, shrub-steppes, } \\
\text { deserts and extreme deserts, } \\
\text { montane vegetation of } \\
\text { Mt. Hermon }\end{array}$ & M \\
\hline Eupatorium cannabinum L. & $\mathrm{ES}, \mathrm{M}, \mathrm{IT}$ & Humid & $\begin{array}{l}\text { Mediterranean woodlands and } \\
\text { shrublands, deserts and } \\
\text { extreme deserts }\end{array}$ & M \\
\hline $\begin{array}{c}\text { Euphorbia hierosolymitana } \\
\text { Boiss. }\end{array}$ & M & Batha, phrygana & $\begin{array}{l}\text { Mediterranean woodlands } \\
\text { and shrublands, } \\
\text { semi-steppe shrublands }\end{array}$ & M \\
\hline Euphorbia graminea Jacq. & $\mathrm{AM}$ & Disturbed habitats & $\begin{array}{l}\text { Mediterranean woodlands } \\
\text { and shrublands }\end{array}$ & M \\
\hline Euphorbia hirsuta L. & M & Humid & $\begin{array}{l}\text { Mediterranean woodlands } \\
\text { and shrublands }\end{array}$ & M \\
\hline Euphorbia terracina L. & M & Sand & $\begin{array}{l}\text { Mediterranean woodlands } \\
\text { and shrublands, } \\
\text { semi-steppe shrublands }\end{array}$ & M \\
\hline $\begin{array}{l}\text { Balanites aegyptiaca } \\
\text { (L.) Delile }\end{array}$ & $S$ & $\begin{array}{l}\text { Deserts, thermophilous } \\
\text { plants }\end{array}$ & $\begin{array}{l}\text { Deserts, shrub-steppes, } \\
\text { semi-steppe shrublands }\end{array}$ & $\mathrm{Tr}, \mathrm{SD}, \mathrm{ED}$ \\
\hline $\begin{array}{l}\text { Zygophyllum dumosum } \\
\text { Boiss. }\end{array}$ & SA & Deserts, shrub-steppes & $\begin{array}{l}\text { Shrub-steppes, semi-steppe } \\
\text { shrublands, deserts }\end{array}$ & $\mathrm{SD}, \mathrm{ED}$ \\
\hline
\end{tabular}


Table 4. Cont.

\begin{tabular}{|c|c|c|c|c|}
\hline Species & Chorotype & Habitat & Distribution & Climate \\
\hline $\begin{array}{c}\text { Nitraria retusa (Forssk.) } \\
\text { Ascherson }\end{array}$ & SA & Salty habitats & $\begin{array}{c}\text { Semi-steppe shrublands, } \\
\text { shrub-steppes, deserts and } \\
\text { extreme deserts }\end{array}$ & $\mathrm{SD}, \mathrm{ED}$ \\
\hline Fagonia bruguieri DC. & SA & $\begin{array}{l}\text { Deserts, thermophilous } \\
\text { plants }\end{array}$ & Desert, shrub-steppes & ED \\
\hline Fagonia mollis Delile & SA & Deserts, shrub-steppes & $\begin{array}{l}\text { Shrub-steppes, semi-steppe } \\
\text { shrublands, Mediterranean } \\
\text { woodlands and } \\
\text { shrublands, deserts }\end{array}$ & $\mathrm{SD}, \mathrm{ED}$ \\
\hline $\begin{array}{c}\text { Fagonia orientalis J. Presl } \\
\text { and C. Presl }\end{array}$ & SA & Sand, deserts & Deserts, shrub-steppes & ED \\
\hline Fagonia arabica L. & SA & Sand & $\begin{array}{l}\text { Deserts, shrub-steppes, } \\
\text { semi-steppe shrublands }\end{array}$ & $\mathrm{SD}, \mathrm{ED}$ \\
\hline $\begin{array}{l}\text { Chenolea arabica (Boiss.) } \\
\text { Diagn. Pl. Orient }\end{array}$ & SA & Salty habitats & $\begin{array}{l}\text { Semi-steppe shrublands, } \\
\text { shrub-steppes, deserts }\end{array}$ & $\mathrm{SD}, \mathrm{ED}$ \\
\hline Convolvulus lanatus Vahl. & SA & Sand & $\begin{array}{l}\text { Deserts, shrub-steppes, } \\
\text { semi-steppe shrublands, } \\
\text { Mediterranean woodlands } \\
\text { and shrublands }\end{array}$ & Tr, SD, ED \\
\hline $\begin{array}{l}\text { Citrullus colocynthis } \\
\text { (L.) Schrader }\end{array}$ & SA & Sand & $\begin{array}{l}\text { Deserts, shrub-steppes, } \\
\text { semi-steppe shrublands, } \\
\text { Mediterranean woodlands } \\
\text { and shrublands }\end{array}$ & Tr, ED \\
\hline Convolvulus dorycnium L. & M & $\begin{array}{c}\text { Bathas and semi-steppe } \\
\text { shrublands }\end{array}$ & $\begin{array}{l}\text { Mediterranean woodlands } \\
\text { and shrublands, } \\
\text { semi-steppe shrublands }\end{array}$ & $\mathrm{M}, \mathrm{Tr}$ \\
\hline Ipomoea cairica (L.) Sweet & $\mathrm{T}$ & Disturbed habitats & $\begin{array}{l}\text { Mediterranean woodlands } \\
\text { and shrublands }\end{array}$ & $\mathrm{M}, \operatorname{Tr}$ \\
\hline $\begin{array}{l}\text { Ipomoea imperati } \\
\text { (Vahl.) Griseb. }\end{array}$ & T, M, ES & Mediterranean strands & $\begin{array}{l}\text { Mediterranean woodlands } \\
\text { and shrublands, } \\
\text { semi-steppe shrublands }\end{array}$ & $\mathrm{M}, \operatorname{Tr}$ \\
\hline Hyoscyamus aureus L. & M, IT & $\begin{array}{l}\text { Cliffs, old walls and } \\
\text { ruins to } 1200 \mathrm{~m}\end{array}$ & $\begin{array}{l}\text { Mediterranean woodlands and } \\
\text { shrublands, semi-steppe } \\
\text { shrublands, shrub-steppes, } \\
\text { deserts and extreme deserts, } \\
\text { montane vegetation } \\
\text { of Mt. Hermon }\end{array}$ & $\mathrm{M}, \mathrm{Tr}, \mathrm{SD}$ \\
\hline $\begin{array}{l}\text { Podonosma orientalis (L.) } \\
\text { Feinbrun, (Podonosma } \\
\text { oriental (L.) Feinbrun, } \\
\text { Podonosma syriacum } \\
\text { (Labill.) Boiss.) }\end{array}$ & M, IT & Hard rock outcrops & $\begin{array}{l}\text { Mediterranean woodlands and } \\
\text { shrublands, semi-steppe } \\
\text { shrublands, shrub-steppes, } \\
\text { deserts and extreme deserts, } \\
\text { montane vegetation } \\
\text { of Mt. Hermon }\end{array}$ & $\mathrm{M}, \mathrm{Tr}, \mathrm{SD}$ \\
\hline $\begin{array}{l}\text { Micromeria myrtifolia } \\
\text { Boiss.et Hohen. }\end{array}$ & M, IT & Hard rock outcrops & $\begin{array}{l}\text { Mediterranean woodlands } \\
\text { and shrublands, } \\
\text { semi-steppe shrublands }\end{array}$ & $\mathrm{M}, \mathrm{Tr}$ \\
\hline $\begin{array}{l}\text { Micromeria nervosa } \\
\text { (Desf.) Benth. }\end{array}$ & M & Hard rock outcrops & $\begin{array}{l}\text { Mediterranean woodlands } \\
\text { and shrublands, } \\
\text { semi-steppe shrublands }\end{array}$ & $\mathrm{M}, \operatorname{Tr}$ \\
\hline $\begin{array}{l}\text { Clinopodium insulare } \\
\text { (Candargy) Govaerts }\end{array}$ & M & Batha, phrygana & $\begin{array}{l}\text { Mediterranean woodlands } \\
\text { and shrublands, } \\
\text { semi-steppe shrublands }\end{array}$ & $\mathrm{M}, \mathrm{Tr}$ \\
\hline Eupatorium cannabinum L. & ES, M, IT & Humid habitats & $\begin{array}{l}\text { Mediterranean woodlands } \\
\text { and shrublands, } \\
\text { deserts and extreme deserts } \\
\text { Semi-steppe shrublands, }\end{array}$ & M \\
\hline Doellia bovei (DC.) Anderb. & SA, S & Humid habitats & $\begin{array}{l}\text { Mediterranean woodlands and } \\
\text { shrublands, deserts }\end{array}$ & M, SD, ED \\
\hline
\end{tabular}


Table 4. Cont.

\begin{tabular}{|c|c|c|c|c|}
\hline Species & Chorotype & Habitat & Distribution & Climate \\
\hline $\begin{array}{l}\text { Helichrysum sanguineum } \\
\text { (L.) Kostel. }\end{array}$ & M & Batha, phrygana & $\begin{array}{l}\text { Mediterranean woodlands } \\
\text { and shrublands, } \\
\text { semi-steppe shrublands }\end{array}$ & M \\
\hline $\begin{array}{l}\text { Iphiona maris-mortui } \\
\text { Feinbrun }\end{array}$ & SA & $\begin{array}{l}\text { Deserts, thermophilic } \\
\text { (heat-loving plants) }\end{array}$ & Characteristics of the salt sea & SD \\
\hline $\begin{array}{c}\text { Chiliadenus iphionoides } \\
\text { (Boiss. and C. I.Blanche) } \\
\text { Brullo. }\end{array}$ & M & $\begin{array}{c}\text { Hard rock outcrops, } \\
\text { steppes, and desert parts }\end{array}$ & $\begin{array}{l}\text { Mediterranean woodlands and } \\
\text { shrublands, semi-steppe } \\
\text { shrublands, shrub-steppes, } \\
\text { deserts and extreme deserts }\end{array}$ & $\mathrm{M}, \mathrm{Tr}, \mathrm{SD}$ \\
\hline $\begin{array}{l}\text { Cota tinctoria (L.) J. Gay. } \\
\text { (Anthemis tinctoria L.) }\end{array}$ & M & Batha, phrygana & $\begin{array}{l}\text { Mediterranean woodlands and } \\
\text { shrublands, Mt. Hermon }\end{array}$ & M \\
\hline Crepis hierosolymitana Boiss. & M & Batha, phrygana & $\begin{array}{l}\text { Mediterranean woodlands and } \\
\text { shrublands, semi-steppe } \\
\text { shrublands, Mt. Hermon }\end{array}$ & M \\
\hline Crepis reuteriana Boiss. & M & $\begin{array}{l}\text { Mediterranean maquis } \\
\text { and forests }\end{array}$ & $\begin{array}{l}\text { Mediterranean woodlands and } \\
\text { shrublands, semi-steppe } \\
\text { shrublands, Mt. Hermon }\end{array}$ & M \\
\hline Cynara syriaca Boiss. & M, IT & Batha, phrygana & $\begin{array}{l}\text { Mediterranean woodlands } \\
\text { and shrublands }\end{array}$ & M \\
\hline $\begin{array}{l}\text { Echinops philistaeus } \\
\text { Feinbrun and Zohary }\end{array}$ & M & Sand & $\begin{array}{l}\text { Semi-steppe shrublands, } \\
\text { Mediterranean woodlands and } \\
\text { shrublands, deserts }\end{array}$ & $\mathrm{M}, \mathrm{Tr}$ \\
\hline Vicia vilosa Roth. & ES, M, IT & Batha, phrygana & $\begin{array}{l}\text { Mediterranean woodlands } \\
\text { and shrublands, } \\
\text { semi-steppe shrublands }\end{array}$ & M \\
\hline $\begin{array}{l}\text { Fumana thymifolia } \\
\text { (L.) Webb. }\end{array}$ & M & Batha, phrygana & $\begin{array}{l}\text { Mediterranean woodlands and } \\
\text { shrublands, semi-steppe } \\
\text { shrublands, shrub-steppes, } \\
\text { deserts and extreme deserts, } \\
\text { Montane vegetation } \\
\text { of Mt. Hermon }\end{array}$ & $\mathrm{M}, \mathrm{Tr}$ \\
\hline Cistus creticus L. & M & Batha, phrygana & $\begin{array}{l}\text { Mediterranean woodlands and } \\
\text { shrublands, semi-steppe } \\
\text { shrublands, shrub-steppes, } \\
\text { montane vegetation } \\
\text { of Mt. Hermon }\end{array}$ & M \\
\hline Galium canum Req. ex DC. & M & Batha, phrygana & $\begin{array}{l}\text { Mediterranean woodlands and } \\
\text { shrublands, semi-steppe } \\
\text { shrublands, shrub-steppes, } \\
\text { montane vegetation } \\
\text { of Mt. Hermon }\end{array}$ & $\mathrm{M}, \mathrm{Tr}$ \\
\hline Galium elongatum C. Presl. & M & Batha, phrygana & $\begin{array}{l}\text { Mediterranean woodlands and } \\
\text { shrublands, semi-steppe } \\
\text { shrublands, shrub-steppes, } \\
\text { montane vegetation } \\
\text { of Mt. Hermon }\end{array}$ & M \\
\hline Galium humifusum M. Bieb. & M, IT & Humid habitats & $\begin{array}{l}\text { Semi-steppe shrublands, } \\
\text { Mediterranean woodlands } \\
\text { and shrublands }\end{array}$ & $\mathrm{M}, \mathrm{Tr}$ \\
\hline Cynanchum acutum L. & M & Batha, phrygana & $\begin{array}{l}\text { Semi-steppe shrublands, Mt. } \\
\text { Hermon, Mediterranean } \\
\text { woodlands and shrublands }\end{array}$ & $\mathrm{M}, \mathrm{Tr}$ \\
\hline Echium glomeratum Poir. & M & Batha, phrygana & $\begin{array}{l}\text { Semi-steppe shrublands, Mt. } \\
\text { Hermon, Mediterranean } \\
\text { woodlands and shrublands }\end{array}$ & M \\
\hline Echiochilon fruticosum Desf. & SA & Sand & $\begin{array}{l}\text { Shrub-steppes, deserts, } \\
\text { semi-steppe shrublands, } \\
\text { Mediterranean woodlands } \\
\text { and shrublands }\end{array}$ & M, Tr, SD, ED \\
\hline
\end{tabular}


Table 4. Cont.

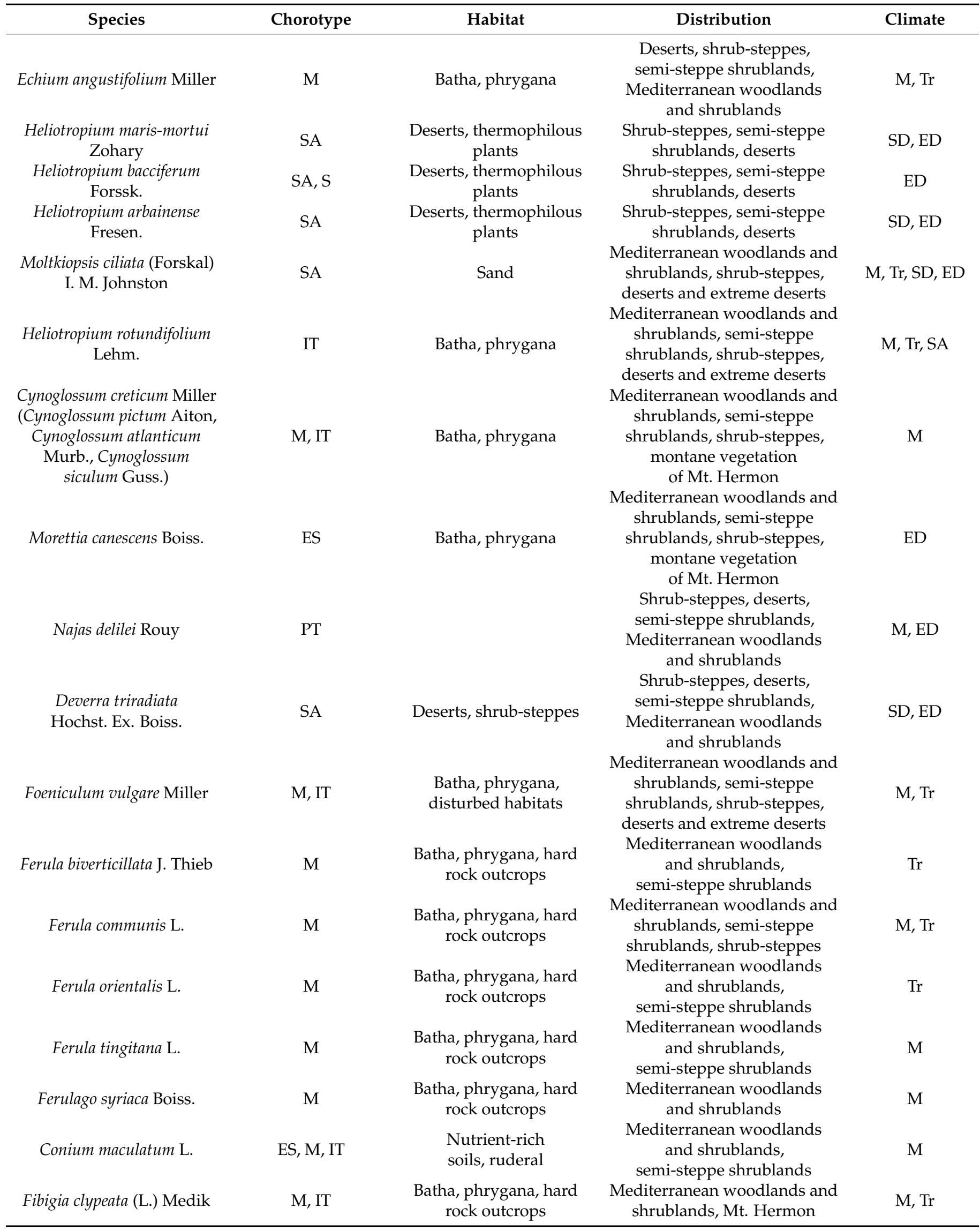


Table 4. Cont.

\begin{tabular}{|c|c|c|c|c|}
\hline Species & Chorotype & Habitat & Distribution & Climate \\
\hline Fibigia eriocarpa (DC.) Boiss. & M & $\begin{array}{l}\text { Mediterranean maquis } \\
\text { and forests }\end{array}$ & $\begin{array}{l}\text { Mediterranean woodlands } \\
\text { and shrublands }\end{array}$ & M \\
\hline $\begin{array}{l}\text { Diplotaxis harra } \\
\text { (Forssk.) Boiss. }\end{array}$ & SA & Deserts, shrub-steppes & $\begin{array}{l}\text { Shrub-steppes, semi-steppe } \\
\text { shrublands, deserts, } \\
\text { Mediterranean woodlands } \\
\text { and shrublands }\end{array}$ & $\mathrm{SD}, \mathrm{ED}$ \\
\hline $\begin{array}{l}\text { Erysimum crassipes } \\
\text { Fisch. and C.A.Mey. }\end{array}$ & IT & Batha, phrygana & $\begin{array}{l}\text { Mediterranean woodlands and } \\
\text { shrublands, semi-steppe } \\
\text { shrublands, shrub-steppes, } \\
\text { deserts and extreme deserts }\end{array}$ & $\mathrm{M}, \mathrm{Tr}$ \\
\hline $\begin{array}{l}\text { Fimbristylis bisumbellata } \\
\text { (Forssk.) Bubani }\end{array}$ & SubT & Humid & $\begin{array}{c}\text { Deserts, semi-steppe } \\
\text { shrublands, Mediterranean } \\
\text { woodlands and shrublands }\end{array}$ & M \\
\hline Forsskaolea tenacissima $\mathrm{L}$. & SA, S & $\begin{array}{l}\text { Deserts, thermophilous } \\
\text { plants }\end{array}$ & $\begin{array}{l}\text { Deserts, semi-steppe } \\
\text { shrublands, Mediterranean } \\
\text { woodlands and shrublands }\end{array}$ & $\mathrm{SD}, \mathrm{ED}$ \\
\hline $\begin{array}{l}\text { Viscum cruciatum } \\
\text { Sieber ex. Boiss. }\end{array}$ & M & $\begin{array}{l}\text { Mediterranean maquis } \\
\text { and forests }\end{array}$ & $\begin{array}{l}\text { Mediterranean woodlands } \\
\text { and shrublands, } \\
\text { semi-steppe shrublands }\end{array}$ & $\mathrm{M}, \mathrm{Tr}$ \\
\hline Glaucium arabicum Fresen. & IT & Shrub-steppes & $\begin{array}{l}\text { Shrub-steppes, deserts, } \\
\text { semi-steppe shrublands, } \\
\text { Mediterranean woodlands } \\
\text { and shrublands }\end{array}$ & SD \\
\hline Glaucium flavum Crantz & M & Mediterranean strands & $\begin{array}{l}\text { Mediterranean woodlands } \\
\text { and shrublands }\end{array}$ & M \\
\hline $\begin{array}{l}\text { Glaucium grandiflorum } \\
\text { Boiss. and A.Huet }\end{array}$ & IT & $\begin{array}{l}\text { Disturbed habitats, } \\
\text { shrub-steppes }\end{array}$ & $\begin{array}{l}\text { Shrub-steppes, deserts, } \\
\text { semi-steppe shrublands, } \\
\text { Mediterranean woodlands } \\
\text { and shrublands }\end{array}$ & $\mathrm{M}, \mathrm{Tr}, \mathrm{SD}, \mathrm{ED}$ \\
\hline Vitex agnus-castus L. & M & Humid & $\begin{array}{c}\text { Deserts, Mediterranean } \\
\text { woodlands and shrublands, } \\
\text { semi-steppe shrublands, } \\
\text { Mt. Hermon }\end{array}$ & $\mathrm{M}, \mathrm{Tr}$ \\
\hline $\begin{array}{l}\text { Globularia arabica } \\
\text { Jaub. and Spach }\end{array}$ & SA & $\begin{array}{l}\text { Batha, phrygana, sand, } \\
\text { hard rock outcrops }\end{array}$ & $\begin{array}{c}\text { Deserts, Mediterranean } \\
\text { woodlands and shrublands, } \\
\text { semi-steppe shrublands, } \\
\text { Mt. Hermon }\end{array}$ & M, SD, ED \\
\hline Hibiscus micranthus L. & $\mathrm{T}$ & Distributes & $\begin{array}{l}\text { Shrub-steppes, Mediterranean } \\
\text { woodlands and shrublands, } \\
\text { semi-steppe shrublands }\end{array}$ & ED \\
\hline Herniaria hemistemon J. Gay & SA & $\begin{array}{l}\text { Deserts, shrub-steppes, } \\
\text { salty habitats }\end{array}$ & $\begin{array}{c}\text { Deserts, Mediterranean } \\
\text { woodlands and shrublands, } \\
\text { semi-steppe shrublands, } \\
\text { Mt. Hermon }\end{array}$ & $\mathrm{SD}, \mathrm{ED}$ \\
\hline Hordeum bulbosum L. & M, IT & Batha, Phrygana & $\begin{array}{c}\text { Shrub-steppes, semi-steppe } \\
\text { shrublands, Mediterranean } \\
\text { woodlands and shrublands, } \\
\text { Mt. Hermon }\end{array}$ & $\mathrm{M}, \mathrm{Tr}$ \\
\hline $\begin{array}{l}\text { Schedonorus arundinaceus } \\
\text { (Schreb.) Dumort. (Festuca } \\
\text { arundinacea Schreb.) }\end{array}$ & $\mathrm{ES}, \mathrm{M}, \mathrm{IT}$ & Humid habitats & $\begin{array}{l}\text { Mediterranean woodlands and } \\
\text { shrublands, Mt. Hermon }\end{array}$ & M \\
\hline $\begin{array}{c}\text { Hypericum triquetrifolium } \\
\text { Turra. }\end{array}$ & M, IT & Batha, phrygana & $\begin{array}{l}\text { Mediterranean woodlands and } \\
\text { shrublands, semi-steppe } \\
\text { shrublands, shrub-steppes, } \\
\text { Montane vegetation } \\
\text { of Mt. Hermon }\end{array}$ & $\mathrm{M}, \mathrm{Tr}$ \\
\hline
\end{tabular}


Table 4. Cont.

\begin{tabular}{|c|c|c|c|c|}
\hline Species & Chorotype & Habitat & Distribution & Climate \\
\hline Aristolochia parvifolia Sm. & M & Batha, phrygana & $\begin{array}{l}\text { Mediterranean woodlands } \\
\text { and shrublands, } \\
\text { semi-steppe shrublands }\end{array}$ & $\mathrm{M}, \mathrm{Tr}$ \\
\hline Iris atrofusca Baker & IT & Batha, phrygana & $\begin{array}{l}\text { Mediterranean woodlands } \\
\text { and shrublands, } \\
\text { semi-steppe shrublands }\end{array}$ & $\mathrm{Tr}, \mathrm{SD}$ \\
\hline Iris atropurpurea Baker & M & Batha, phrygana & $\begin{array}{l}\text { Mediterranean woodlands } \\
\text { and shrublands }\end{array}$ & M \\
\hline Iris palaestina (Bak.) Boiss. & M & Batha, phrygana & $\begin{array}{c}\text { The sandstone ridges of the } \\
\text { coastal plain }\end{array}$ & $\mathrm{M}, \mathrm{Tr}$ \\
\hline Iris vartanii Foster & M & Batha, phrygana & $\begin{array}{l}\text { Mediterranean woodlands } \\
\text { and shrublands, } \\
\text { semi-steppe shrublands }\end{array}$ & $\mathrm{M}, \mathrm{Tr}$ \\
\hline Gladiolus italicus Miller & M, IT & Batha, phrygana & $\begin{array}{l}\text { Mediterranean woodlands } \\
\text { and shrublands, } \\
\text { semi-steppe shrublands }\end{array}$ & $\mathrm{M}, \mathrm{Tr}$ \\
\hline $\begin{array}{c}\text { Juncus acutus L. } \\
\text { (Juncus littoralis C.A.Mey.) } \\
\text { (Juncus spinosus Forssk.) }\end{array}$ & M, IT & Humid habitats & $\begin{array}{l}\text { Mediterranean woodlands and } \\
\text { shrublands, semi-steppe } \\
\text { shrublands, shrub-steppes, } \\
\text { deserts and extreme deserts }\end{array}$ & $\mathrm{M}, \mathrm{Tr}$ \\
\hline Juncus articulates L. & ES, M, IT & Humid habitats & $\begin{array}{l}\text { Mediterranean woodlands } \\
\text { and shrublands, } \\
\text { semi-steppe shrublands }\end{array}$ & M \\
\hline Juncus subulatus Forssk. & M & Humid habitats & $\begin{array}{l}\text { Semi-steppe shrublands, } \\
\text { shrub-steppes, Mediterranean } \\
\text { woodlands and shrublands }\end{array}$ & M \\
\hline $\begin{array}{l}\text { Jacquemontia unilateralis } \\
\text { (Roem. and Schult.) } \\
\text { O'Donell }\end{array}$ & M & Batha, phrygana & $\begin{array}{l}\text { Shrub-steppes, deserts, } \\
\text { semi-steppe shrublands, } \\
\text { Mediterranean woodlands } \\
\text { and shrublands }\end{array}$ & M \\
\hline $\begin{array}{l}\text { Kickxia aegyptiaca } \\
\text { (L.) Nabelek }\end{array}$ & M, SA & Batha, phrygana, deserts & $\begin{array}{l}\text { Shrub-steppes, deserts, } \\
\text { semi-steppe shrublands, } \\
\text { Mediterranean woodlands } \\
\text { and shrublands }\end{array}$ & $\mathrm{M}, \mathrm{Tr}$ \\
\hline Kickxia judaica Danin & SA & $\begin{array}{l}\text { Hard rock outcrops, } \\
\text { shrub-steppes }\end{array}$ & $\begin{array}{l}\text { Semi-steppe shrublands, } \\
\text { Mediterranean woodlands } \\
\text { and shrublands }\end{array}$ & $\mathrm{Tr}, \mathrm{SD}$ \\
\hline $\begin{array}{l}\text { Launaea nudicaulis (L.) } \\
\text { Hooker fil. }\end{array}$ & SA & $\begin{array}{l}\text { Deserts, thermophilous } \\
\text { plants }\end{array}$ & $\begin{array}{c}\text { Shrub-steppes, deserts, } \\
\text { semi-steppe shrublands, } \\
\text { Mediterranean woodlands } \\
\text { and shrublands }\end{array}$ & SD, DX \\
\hline Leontodon tuberosus L. & M & Batha, phrygana & $\begin{array}{l}\text { Semi-steppe shrublands, } \\
\text { Mediterranean woodlands and } \\
\text { shrublands, Mt. Hermon }\end{array}$ & $\mathrm{M}, \mathrm{Tr}$ \\
\hline $\begin{array}{l}\text { Leopoldia bicolor (Boiss.) } \\
\text { Eig. and Feinbrun }\end{array}$ & M & Sand & $\begin{array}{l}\text { Mediterranean woodlands } \\
\text { and shrublands }\end{array}$ & M \\
\hline $\begin{array}{l}\text { Leptadenia pyrotechnica } \\
\text { (Forssk.) Decne. }\end{array}$ & SA, S & $\begin{array}{l}\text { Deserts, thermophilous } \\
\text { plants }\end{array}$ & Deserts & ED \\
\hline Fimbristylis ferruginea (L.) & $\mathrm{T}$ & Humid habitats & $\begin{array}{c}\text { Mediterranean woodlands } \\
\text { and shrublands }\end{array}$ & M \\
\hline Casuarina equesitifolia L. & M, IT, SA & Light soils, deserts & $\begin{array}{l}\text { Mediterranean woodlands and } \\
\text { shrublands, semi-steppe } \\
\text { shrublands, deserts }\end{array}$ & $\mathrm{M}, \mathrm{Tr}, \mathrm{SD}, \mathrm{ED}$ \\
\hline
\end{tabular}

Abbreviations: Chorotype: M (Mediterranean), OM (Oro-Mediterranean), IT (Irano-Turanian), SA (SaharoArabian), S (Sudanian), SUA (Sudanian African), A (American), ES (Euro-Siberian), EP-EC (exotic, planted, escaped from cultivation), EC (escaped from cultivation), T (Tropical), PT (Plurireginalbor-trop) and SubT (subtropical-tropical). Climate region: M (Mediterranean), D (deserts), Tr. (transition), SD and ED (semi and extreme deserts), DX (desert mixed). Mt. Hermon: Mount Hermon. 
Although Beit Jibrin rises slightly above sea level, it represents a unique pattern of forest vegetation and biodiversity. It is rich in endemic plants, which are estimated to account for about 37 (12.75\%) endemic species of the total plants, home to more than 290 species of plants, including forests, oak, steppes, copses and high shrub lands. Therefore, they are part of the mountain highland plants, as in the highlands that extend from the southernmost point of Hebron to the north of Palestine, such as Jenin, Safed and Galilee; Palestinian coast plants and the Mediterranean basin region, such as Jabal Al-Sheikh, Jaffa, Acre, Haifa, Nazareth and Ashdod; savannah plants; and other African desert plants, such as Sinai and the Red Sea area. However, Beit Jibrin represents forest plants found in West Asia, the Mediterranean region, North Africa and the Palestinian coast. Beit Jibrin has an infra-thermomediterranean thermotype and a dry ombrotype. In this study, two new plant groups were identified in the Beit Jibrin area: Cupresso sempervirentis-Pinetum halepensis ass. nova and Pistacio lentisci-Quercetum calliprini ass. nova.

The suggested syntaxonomical scheme for this study is:

Class: Quercetea ilicis Br.-Bl. ex. A. and O. Bolòs 1950 [69]

Order: Pinetalia halepensis Biondi et al. (2014) [27]

Alliance: Cupresso sempervirentis_Pinus halepensis all. nova

Cupresso sempervirentis-Pinetum halepensis ass. nova

Class: Quercetea ilicis Br.-Bl. ex. A. and O. Bolòs 1950 [62]

Class: Quercetea calliprini or palaestini nova.

Order: Quercetalia calliprini Zohary 1960 [64]

Alliance: Quercion calliprini Zohary 1955, 1960 [63,64]

Pistacio lentisci-Quercetum calliprini ass. nova

Syntaxonomical scheme:

Class: Quercetea ilicis Br.-Bl. ex A. Bolòs et O. de Bolòs in A. Bolòs y Vayreda 1950 [69]

Order: Quercetalia ilicis Br.-Bl. ex Molinier 1934 [70]

Quercetalia calliprini Zohary 1955, 1960 [63,64]

Alliance: Ceratonio-Pistacion lentisci Zohary ex Zohary et Orshan 1959 [74]

Associations:

-Pistacio palaestinae-Quercetum lokii* (Ighbareyeh et al., 2014) [16]

-Capparido sinaicae-Ceratonietum siliquae (Ighbareyeh et al., 2014) [16]

-Cerasus microcarpae-Quercetum ithaburensis * (Ighbareyeh et al., 2014) [16]

-Pyro siriacae-Abietetum cilicicae * (Ighbareyeh et al., 2014) [16]

-Abio ciliciae-Ceratonietum siliquae (Ighbareyeh et al., 2014) [16]

-Periploco aphylli-Pinetum halepensis (Ighbareyeh et al., 2014) [16]

-Cytisopsis pseudocytiso-Tamaricetum tetragynae (Ighbareyeh et al., 2014) [16]

-Crataego sinaicae-Tamaricetum jordanii (Ighbareyeh et al., 2014) [16]

Class: Quercetea ilicis Br.-Bl. ex A. Bolòs et O. de Bolòs in A. Bolòs y Vayreda 1950 [62]

Order: Quercetalia calliprini Zohary 1955, 1960 [62,63]

Alliance: Ceratonio-Pistacion lentisci Zohary ex Zohary et Orshan 1959 [74]

Associations:

-Pino halepensis-Quercetum lookii* (Ighbareyeh et al., 2018) [75]

-Pistacio palaestinae-Ceratonietum siliquae*(Ighbareyeh et al., 2018) [75]

-Quercus libanii-Tamaricetum palaestineae* (Ighbareyeh et al., 2018) [75]

Class: Quercetea ilicis Br.-Bl. ex A. Bolòs et O. de Bolòs in A. Bolòs y Vayreda 1950 [62]

Order: Quercetalia calliprini Zohary 1955, 1960 [63,64]

Alliance: Pistacio-Quercion lokii (Ighbareyeh et al., 2021) [19]

Ceratonio siliquae-Quercion calliprinae (Ighbareyeh et al., 2021) [19]

Pino halepensis-Cupression sempervirenti (Ighbareyeh et al., 2021) [19]

Associations:

-Pistacio lentisci-Quercetum lokii (Ighbareyeh et al., 2021) [19]

-Ceratonio siliquae-Quercetum callipinii. (Ighbareyeh et al., 2021) [19]

-Pino halepensis-Cupressetum sempervirentis (Ighbareyeh et al., 2021) [19]

* Associations in which olive cultivation is possible. 


\begin{abstract}
Author Contributions: Conceptualization: J.M.H.I. and E.C.; data curation: J.M.H.I., A.C.-O., and E.C.; formal analysis: E.C.; investigation: J.M.H.I. and E.C.; methodology: E.C. and A.C.-O.; project administration: E.C.; resources: E.C.; supervision: J.M.H.I., A.C.-O. and E.C.; validation, J.M.H.I., A.C.-O. and E.C.; software: J.M.H.I. and E.C.; visualization: E.C., A.C.-O. and J.M.H.I..; writingoriginal draft: J.M.H.I. and E.C.; writing—review and editing: J.M.H.I., A.C.-O., and E.C. All authors have read and agreed to the published version of the manuscript.
\end{abstract}

Funding: This work does not have funds for its publication, only a small help from the University of Jaén, Spain.

Acknowledgments: This work is the result of the first author's doctoral thesis, directed at the University of Jaén.

Conflicts of Interest: The authors declare no conflict of interest.

\title{
References
}

1. Jehad, M.H.I.; Suliemieh, A.A.A.; Ighbareyeh, M.M.H.; Cano-Carmoma, E.; Cano-Ortiz, A. Olive (Olea europaea L.) of Jerusalem in Palestine. Trends Tech. Sci. Res. 2019, 3, 555617. [CrossRef]

2. Ighbareyeh, J.M.H.; Cano-Ortiz, A.; Cano, E. Case study: Analysis of the physical factors of Palestinian bioclimate. Am. J. Clim. Chang. 2014, 3, 223-231. [CrossRef]

3. Ighbareyeh, J.M.H. Effect of environmental factors on Apricot (Prunus armeniaca L.) yield in the city of Jerusalem occupied, Palestine. Asian J. Res. Agric. For. 2021, 7, 12-24. [CrossRef]

4. Zohary, M. Plant Life of Palestine; Ronald Press Company: New York, NY, USA, 1962.

5. Zohary, M. Flora Palaestina 1966; Israel Academy of Sciences and Humanities: Jerusalem, Israel, 1966; Volume I.

6. Zohary, M. Flora Palaestina. Part 1, Text Equisetaceae to Moringaceae; Israel Academy of Science and Humanities: Jerusalem, Israel, 1966; p. 346.

7. Zohary, M. Flora Palaestina 1972; Israel Academy of Sciences and Humanities: Jerusalem, Israel, 1972; Volume II.

8. Zohary, M. Geobotanical Foundations of the Middle East; Gustav Fisher Verlag: Stuttgart, Germany, 1973; Volumes I and II, p. 739.

9. Zohary, M. Flora Palaestina 1986; Israel Academy of Sciences and Humanities: Jerusalem, Israel, 1986; Volume IV.

10. Zohary, M. Flora Palaestina 1987; Israel Academy of Sciences and Humanities: Jerusalem, Israel, 1987; Volume III.

11. Dothan, F.N. Flora Palaestina, Part Three, Text Ericaceae to Compositae; Israel Academy of Science and Humanities: Jerusalem, Israel, 1978; p. 481.

12. Dothan, F.N. Flora Palaestina, Part Four Plates, Text Alismtaceae to Orchidaceae; Academy of Science and Humanities: Jerusalem, Israel, 1986; p. 525.

13. Danin, A.; Feinbrun-Dothan, N. Analytical Flora of Eretz-Israel; CANA Publishing House Ltd.: Jerusalem, Israel, 1991.

14. Danin, A. The inclusion of adventive plants in the second edition of Flora Palaestina. Willdenowia 2004, 30, 305-314. [CrossRef]

15. Danin, A. Distribution Atlas of Plants in Flora Palaestina Area, 2nd ed.; Academy of Science and Humanities: Jerusalem, Israel, 2004; p. 520. ISBN 9652081671.

16. Ighbareyeh, J.M.H.; Cano-Ortiz, A.; Suliemieh, A.A.A.; Ighbareyeh, M.M.H.; Cano, E. Phytosociology with other characteristic biologically and ecologically of plant in Palestine. Am. J. Plant Sci. 2014, 5, 3104-3118. [CrossRef]

17. Ighbareyeh, J.; Cano-Ortiz, A.; Carmona, E.; Suliemieh, A.; Ighbareyeh, M. Flora endemic rare and bioclimate of Palestine. Open Access Libr. J. 2017, 4, 1-14. [CrossRef]

18. Ighbareyeh, J.M.H.; Cano-Ortiz, A.; Cano, E. Endemic plant species in the west of Hebron, Palestine. Eur. J. Appl. Sci. 2021, 9 , 368-385. [CrossRef]

19. Ighbareyeh, J.M.H.; Suliemieh, A.A.-R.A.; Abu Ayash, A.M.; Sheqwara, M.N.; Ortiz, A.C.; Carmona, E.C. Biodiversity and phytosociological analysis of plants in wadi Al-Quf nursery reserve North-Western of Hebron City in Palestine. J. Plant Sci. 2021, 9, 13. [CrossRef]

20. Ercanlı, I.; Günlü, A.; Şenyurt, M.; Keleş, S. Artificial neural network models predicting the leaf area index: A case study in pure even-aged Crimean pine forests from Turkey. For. Ecosyst. 2018, 5, 29. [CrossRef]

21. Pérez-Latorre, A.V.; Navas, P.; Navas, D.; Gil, Y.; Cabezudo, B. Datos sobre la flora y la vegetación de la Serranía de Ronda (Málaga, España). Acta Bot. Malacit. 1998, 23, 149-191. [CrossRef]

22. Pérez-Latorre, A.V.; Navas-Fernández, D.; Gavira, O.; Caballero, G.; Cabezudo, B. Vegetación del Parque Natural de Las Sierras Tejeda, Almijara y Alhama (Málaga-Granada, España). Acta Bot. Malacit. 2004, 29, 117-190. [CrossRef]

23. Mendes, P.; Meireles, C.; Vila-Viçosa, C.; Musarella, C.M.; Pinto-Gomes, C. Best management practices to face degraded territories occupied by Cistus ladanifer shrublands-Portugal case study. Plant Biosyst. Int. J. Deal. Asp. Plant Biol. 2013, 149, 494-502. [CrossRef]

24. Pérez-García, F.J.; Akhani, H.; Parsons, R.F.; Silcock, J.L.; Kurt, L.; Özdeniz, E.; Spampinato, G.; Musarella, C.M.; Sánchez, E.S.; Sola, F.; et al. A first inventory of gypsum flora in the Palearctic and Australia. Mediterr. Bot. 2018, 39, 35-49. [CrossRef] 
25. Bartolucci, F.; Peruzzi, L.; Galasso, G.; Albano, A.; Alessandrini, A.; Ardenghi, N.M.G.; Astuti, G.; Bacchetta, G.; Ballelli, S.; Banfi, E.; et al. An updated checklist of the vascular flora native to Italy. Plant Biosyst. Int. J. Deal. Asp. Plant Biol. 2018, 152, 179-303. [CrossRef]

26. Musarella, C.M.; Mendoza-Fernández, A.J.; Mota, J.F.; Alessandrini, A.; Bacchetta, G.; Brullo, S.; Caldarella, O.; Ciaschetti, G.; Conti, F.; Di Martino, L.; et al. Checklist of gypsophilous vascular flora in Italy. PhytoKeys 2018, 103, 61-82. [CrossRef]

27. Biondi, E.; Blasi, C.; Allegrezza, M.; Anzellotti, I.; Azzella, M.M.; Carli, E.; Casavecchia, S.; Copiz, R.; Del Vico, E.; Facioni, L.; et al. Plant communities of Italy: The vegetation prodrome. Plant Biosyst. Int. J. Deal. Asp. Plant Biol. 2014, 148, 728-814. [CrossRef]

28. Galasso, G.; Conti, F.; Peruzzi, L.; Ardenghi, N.M.G.; Banfi, E.; Celesti-Grapow, L.; Albano, A.; Alessandrini, A.; Bacchetta, G.; Ballelli, S.; et al. An updated checklist of the vascular flora alien to Italy. Plant Biosyst. Int. J. Deal. Asp. Plant Biol. 2018, 152, 556-592. [CrossRef]

29. Khalidi, W. All That Remains: The Palestinian Villages Occupied and Depopulated by Israel in 1948; Institute for Palestine Studies: Washington, DC, USA, 1992; ISBN 0-88728-224-5.

30. Hadawi, S. Village statistics of 1945: A classification of land and area ownership in Palestine. Palest. Lib. Organ. Res. Cent. 1970, 34,30 .

31. Abu-Sitta, S. The Return Journey; Palestine Land Society: London, UK, 2007; ISBN 0-9549034-1-2.

32. UNESCO World Heritage Centre. Region of the Caves \& Hiding: Bet Guvrin-Maresha Archived 2017. Available online: https: / / whc.unesco.org/en/list/1370/ (accessed on 27 October 2017).

33. Braun-Blanquet, J. Pflanzensoziologie. Grundzüge der Vegetationskunde, 3rd ed.; Springer: Vienna, Austria, 1964; p. 631. [CrossRef]

34. Braun-Blanquet, J. Fitosociología. Bases para el Estudio de las Comunidades Vegetales; Blume: Madrid, Spain, $1979 ;$ p. 820.

35. Van Der Maabel, E. Transformation of cover-abundance values in phytosociology and its effects on community similarity. Plant Ecol. 1979, 39, 97-114. [CrossRef]

36. Braun-Blanquet, J.; Bolòs, O.D. Les groupements végétaux du bassin moyen de l'Ebre et leur dynamisme. An. Estac. Exp. Aula Dei 1957, 5, 1-266.

37. Weber, H.; Moravec, J.; Theurillat, J.-P. International code of phytosociological nomenclature. J. Veg. Sci. 2000, 11, 739-768. [CrossRef]

38. Theurillat, J.-P.; Willner, W.; Fernández-González, F.; Bültmann, H.; Čarni, A.; Gigante, D.; Mucina, L.; Weber, H. International code of phytosociological nomenclature. Appl. Veg. Sci. 2020, 24. [CrossRef]

39. Bolòs, O.; De Molinier, R. Recherches phytosociologiques dans l'île de Majorque. Collect. Bot. 1958, 34, $699-865$.

40. Oakley, K.P. The Excavation of Goarham's Cave. Gibraltar 1951-1954. Bull. Inst. Archaelogy 1958, 4, 1-219.

41. Bolòs, O.; De Vigo, J.; Masalles, R.M.; Ninot, J.M. Manual dels Paisos Catalans; Portic: Barcelona, Spain, 1990.

42. Pott, R. Phytosociology: A modern geobotanical method. Plant Biosyst. Int. J. Deal. Asp. Plant Biol. 2011, 145, 9-18. [CrossRef]

43. Biondi, E. Phytosociology today: Methodological and conceptual evolution. Plant Biosyst. Int. J. Deal. Asp. Plant Biol. 2011, 145, 19-29. [CrossRef]

44. Rivas-Martínez, S.; Cantó, P.; Fernández-González, F.; Sánchez-Mata, D. Revision de la clase Quercetea ilicis en Espana y Portugal: 1. Subalianza Quercenion ilicis. Folia Bot. Matrit. 1995, 15, 1-20.

45. Rivas-Martínez, S. Clasificación bioclimática de la Tierra. Folia Bot. Matritensis 1996, 16, 1-20.

46. Rivas-Martinez, S.; Sanchez, M.D.; Costa, M. North American boreal and western temperate forest vegetation (Syntaxonomical synopsis of the potential natural plant communities of North America, II. Itinera Geobot. 1999, 12, 5-316.

47. Rivas-Martínez, S.; Fernández-González, F.; Loidi, J.; Lousã, M.; Penas, A. Syntaxonomical checklist of vascular plant communities of Spain and Portugal to association level. Itinera Geobot. 2001, 14, 5-341.

48. Rivas-Martínez, S.; Díaz, T.E.; Fernández-González, F.; Izco, J.; Lousã, M.; Penas, A. Vascular plant communities of Spain and Portugal. Addenda to the syntaxonomical checklist of 2001. Itinera Geobot. 2002, 15, 5-432.

49. Rivas-Martínez, S.; Biondi, E.; Costa, M.; Mossa, L. Datos sobre la vegetación de la clase Quercetea ilicis en Cerdena. Fitosociologia 2003, 40, 35-38.

50. Rivas-Martınez, S.; Rivas Saenz, S.; Penas, A. Worldwide bioclimatic classification system. Glob. Geobot. 2011, 1, 1-634.

51. Rivas-Martínez, S.; Penas, A.; del Río, S.; Díaz, G.T.; Rivas-Sáenz, S. Bioclimatology of the Iberian Peninsula and the Balearic Islands. In The Vegetation of the Iberian Peninsula; Loidi, J., Ed.; Springer: Vienna, Austria, 2017; pp. $29-80$.

52. Panetsos, C.P. Natural hybridization between Pinus halepensis and Pinus brutia. Silvae Genet. 1975, $24,163-168$.

53. Grandos, M.; Martín-Vicente, A.; García Novo, F. Introducción del Pinus pinea en el Parque Natural de Doñana. En Actas del Seminario Sobre Reservas de la Biosfera; La Rábida: Huelva, Spain, 1983.

54. Fernández-Galiano, E. Pasado, presente y futuro de los boques de la Península Ibérica. Acta Bot. Malacit. 1990, 15, 135-143. [CrossRef]

55. Gil, L.; Aranzazu, M.; Gordo, J.; De Miguel, J.; Mutke, S.; Catalán-Bachiller, G.; Iglesias, S. Las Regiones Procedencia de Pinus pinea L; Ministerio de Medio Ambiente: Madrid, Spain, 1997.

56. GIL, L. Consideraciones históricas sobre "Pinus pinaster"Aiton en el paisaje vegetal de la península ibérica. Estudios Geográficos $1991,52,5$.

57. Martínez-Montes, E.; Alejandro, M.M.R.; Villalón-Torresn, D. Los pinares de pino piñonero en el sur peninsular. Papel en la dinámica natural en base a la arqueología prehistórica y protohistórica. Nuevas interpretaciones. Cuad. Soc. Esp. Cien. For. 2003, $16,121-126$. 
58. Burrascanno, S.; Rosati, L.; Blasi, C. Plant species diversity in Mediterranean old-growth forests: A case study from central Italy. Plant Biosyst. 2009, 143, 190-200. [CrossRef]

59. Farjon, A. Biodiversity of Pinus (Pinaceae) in Mexico: Speciation and palaeo-endemism. Bot. J. Linn. Soc. 1996, 121, 365-384. [CrossRef]

60. Pérez-Latorre, A.V.; Casimiero, F.; García-Sánchez, J.; Cabezudo, B. Flora y vegetación del Paraje Natural Desfiladero de los Gaitanes y su entorno (Málaga). Acta Bot. Malacit. 2014, 39, 129-177. [CrossRef]

61. Pérez-Latorrre, A.V.; Casimiro, F.; Cabezudo, B. Flora y vegetación de la sierra de Alcaparaín (Málaga, España). Acta Bot. Malacit. 2015, 40, 107-156. [CrossRef]

62. Pesaresi, S.; Bioindi, E.; Vagge, I.; Galdenzi, D.; Casavecchia, S. The Pinus halepensis Miller Forests in the central-eastern European Mediterranean basin. Plant Biosyst. 2017, 151, 512-529. [CrossRef]

63. Zohary, M. Geobotany; Sifriyat Poalim Ltd.: Maanit, Israel, 1955; 590p. (In Hebrew)

64. Zohary, M. The maquis of Quercus calliprinos in Israel and Jordan. Bull. Res. Counc. Isr. 1960, 9, 51-72.

65. Ezra-Barnea. The Israel Oak Registry, International Oak Society Blog 2018. Available online: https: / / www.internationaloaksociety. org/content/israel-oak-registry\#_ftnref1 (accessed on 20 November 2021).

66. Molero, M.J.; Pérez-Raya, F. Estudio fitosociológico de los sabinares de Juniperus phoenicea L. en el sector Malacitano-Almijarense (provincia corológica Bética). Lazaroa 1987, 7, 301-306.

67. Bonari, G.; Fernández-González, F.; Çoban, S.; Monteiro-Henriques, T.; Bergmeier, E.; Didukh, Y.P.; Xystrakis, F.; Angiolini, C.; Chytrý, K.; Acosta, A.T.; et al. Classification of the Mediterranean lowland to submontane pine forest vegetation. Appl. Veg. Sci. 2021, 24, e12544. [CrossRef]

68. Gil, L. Las Transformaciones Históricas del Paisaje: La Permanencia y la Extinción Local del Pino Piñonero. Los Montes y su Historia. Una Perspectiva Política, Económica y Social; Universidad de Huelva: Huelva, Spain, 1999; pp. 151-186.

69. Braun-Blanquet, J.; de Bolòs, O. Aperçu des Groupements Végétaux des Montagnes tarragonaises. Collect. Bot 1950, 2, 303-342.

70. Molinier, R. Études phytosociologiques et écologiques en Provence occidentale. An. Mus. Hist. Nat. Marseille 1934, $27,1-273$.

71. Rivas-Martínez, S. La vegetacion de la clase Quercetea ilicis en Espana y Portugal. An. Inst. Bot. Cavanilles 1975, 31, $205-259$.

72. Rivas-Martínez, S. Sobre la nueva clase Polygono-Poetea annuae. Phytocoenologia 1975, 2, 123-140. [CrossRef]

73. Tsiouvaras, C.N. Ecology and management of Kermes Oak (Quercus coccifera L.) Shrublands in Greece: A review. J. Range Manag. 1987, 40, 542. [CrossRef]

74. Zohary, M.; Orshan, G. The maquis of ceratonia siliqua in Israel. Vegetatio 1959, 8, 285-297. [CrossRef]

75. Ighbareyeh, J.M.H.; Carmona, E.C. A phytosociological of plant communities and Biodiversity in the East-South of Idna Village-Hebron of Palestine. Int. J. Geosci. 2018, 9, 44-58. [CrossRef] 